\title{
Plant Species of Special Concern and \\ Plant Associations of Sheridan County, Montana
}

\author{
Prepared for: \\ U.S. Fish and Wildlife Service \\ Prepared by: \\ Bonnie Heidel, Stephen V. Cooper, and Catherine Jean
}

September, 2000

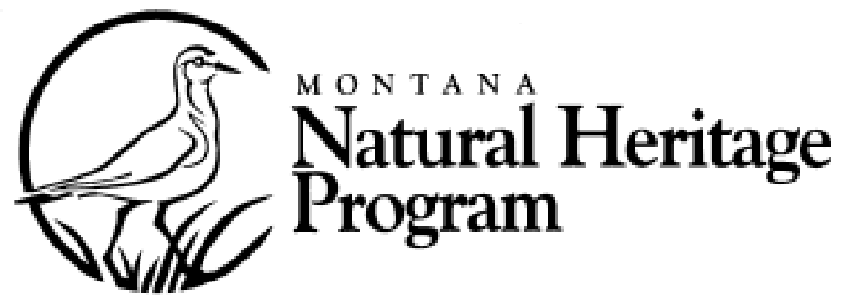




\section{Plant Species of Special Concern and Plant Associations of Sheridan County, Montana}

(C) 2000 Montana Natural Heritage Program

State Library Building • P.O. Box 201800 • 1515 East Sixth Avenue • Helena, MT 59620-1800 • 406-444-3009

USFWS Agreement Number 1448-60181-98-J378

Task Order Number 16

U. S. Fish and Wildlife Service

100 North Park,Suite 320

Helena, Montana 59601

This document should be cited as follows:

Heidel, B., S. V. Cooper, and C. Jean. 2000. Plant species of special concern and plant associations of Sheridan County, Montana. Report to the U.S. Fish and Wildlife Service. Montana Natural Heritage Program, Helena. 22 pp. plus appendices. 


\section{Acknowledgements}

This report is dedicated to the memory of our esteemed colleague, Jim Reichel, who had initiated a biological inventory within the study area at the time of his tragic passing.

The expertise and interest of U.S. Fish and Wildlife Service staff was integral to this project, which greatly benefited by the coordination and expertise of Mike Rabenberg. It also benefited from discussions and help of the entire Medicine Lake Office.

The access permission and kindness of landowners and leasees contacted in this study are acknowledged with gratitude. Special thanks are also extended to all who provided information on the plant life of Sheridan County, or the county in general, including Mike Rabenberg (USFWS), Aldon Joyes, Doug Smith, Cherryl Wagner, Ted Nordhagen, Monica Friedrich (NRCS) and Terry Angvick (Extension Service).
Duane Lund (Natural Resource Information Service) designed the shaded relief map. Cedron Jones (Montana Natural Heritage Program) created all other maps. Martin Miller processed plant association and species records. John Hinshaw scanned images and provided system support. Ryan Rauscher prepared the climate graph. Debra Tirmenstein computerized the plot data. Margaret Beer professionally formatted the final report and helped orchestrate all of the above-mentioned work. Sue Crispin contributed expert editing. The report also reflects the interest and technical zoology support of Paul Hendricks. This work benefited from the contributions of many people, while any shortcomings rest with the authors.

This project was supported by a challenge costshare agreement between the U.S. Fish and Wildlife Service and the Montana Natural Heritage Program. 


\section{Executive Summary}

Inventory was conducted in Sheridan County for plant associations and plant species of special concern. Forty-two plant associations are known from the county, of which five are described for the first time, and another nine are documented for the first time in Montana, in addition to information gathered on 15 undescribed types that are under review. They include highly productive grasslands, wet meadows, and sandhill plant associations that are significant within Montana, and may be of significance in the United States or the Great Plains as a whole.

Fifteen plant species of special concern or watch species are known from the county, of which eight were documented for the first time, and one is restricted to the county. Almost all of the plant species of special concern are restricted to wet meadow or sandy habitats. For almost half of the 15 species, Sheridan County populations represent the largest known population in Montana. They are rare strictly from a state perspective, though some may be vulnerable to rangewide decline.

This study also identified areas of high quality vegetation and concentrations of different plant species of special concern and plant associations. The Missouri Coteau and the Medicine Lake areas at the eastern end of Sheridan County have the greatest diversity of high quality plant associations and the highest numbers and concentrations of special concern plant species in the county, in part because they encompass the most extensive and diverse wetland habitats. Upper reaches of the Big Muddy valley and tributaries are also intact in places and significant for their plant associations.
The alkali lake systems, sandhills, and prairie pothole mosaic of wetlands and grasslands in Sheridan County are some of the largest identified in eastern Montana. Range management, noxious weed control, and related grassland/wetland conservation measures are important to their longterm management.

The compiled information is offered as a reference to:

- Identify management opportunities for maintaining rare species and high quality natural vegetation on public lands,

- Re-evaluate plant species and natural vegetation types as they do or do not actually warrant consideration as features of special concern, and

- Profile biological features and areas unique to the county for consideration in watershed coordination and countywide planning. 


\section{T able of Contents}

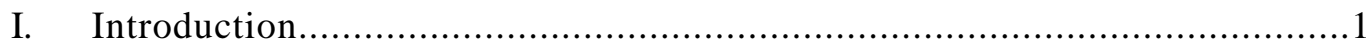

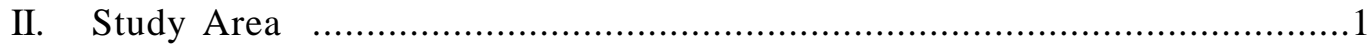

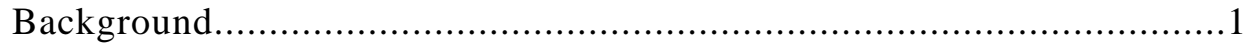

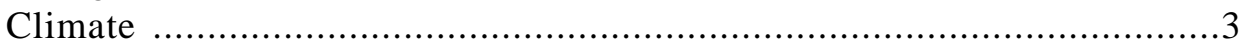

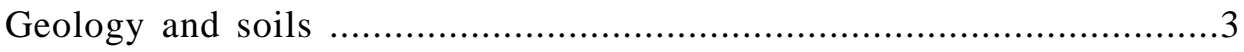

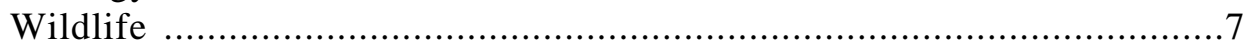

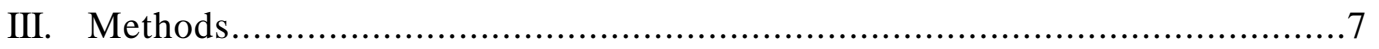

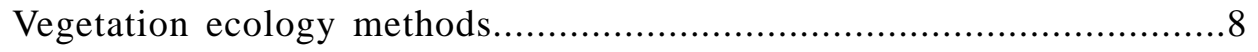

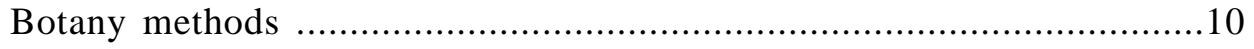

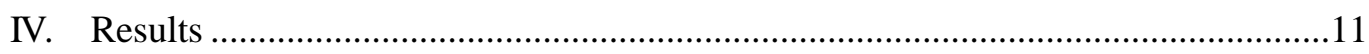

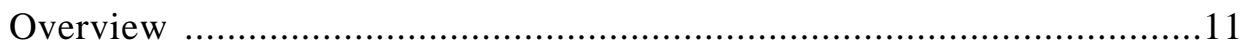

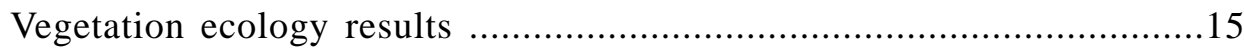

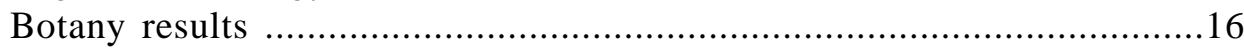

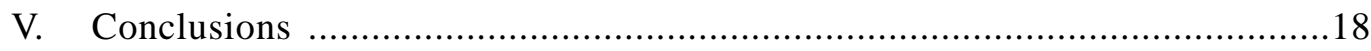

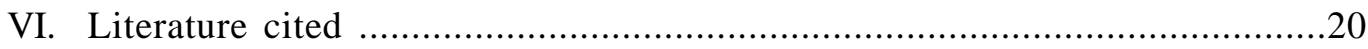

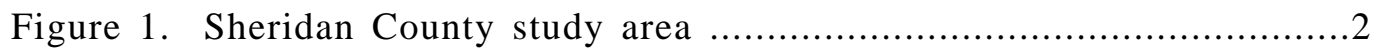

Figure 2. Topography of Sheridan County area ..................................5

Figure 3. Climate of Plentywood, Montana .....................................6

Figure 4. Maximum extent of continental ice margins in the Great Plains..6

Figure 5. Vegetation study areas in Sheridan County .........................

Figure 6. Photographs of the Medicine Lake area .............................. 12

Figure 7. Photographs of the Missouri Coteau prairie pothole area ..........13

Figure 8. Photographs of the Big Muddy headwaters area .....................14

Figure 9. Plant species of special concern in Sheridan County..................17

Appendix A. Animal species of special concern in Sheridan County

Appendix B. Equivalent scientific names for characteristic plants in Sheridan County

Appendix C. Plant species inventory targets in Sheridan County

Appendix D. Summary table of Sheridan County plant associations

Appendix E. Sheridan County plant associations

Appendix F. Characteristic plant associations of Sheridan County

Appendix G. Range sites, ecological units, and plant associations of Sheridan County

Appendix H. Summary table of Sheridan County plant species of special concern and watch species

Appendix I. Sheridan County plant species of special concern and watch species

Appendix J. Flora of Sheridan County 


\section{Introduction}

\section{0 bjectives}

The purpose of this study was to conduct a systematic inventory of plant species of special concern and plant associations that represent the natural vegetation of Sheridan County. It serves to:

- Identify management opportunities for maintaining rare species and high quality natural vegetation on public lands,

- Re-evaluate plant species and natural vegetation types as they do or do not actually warrant consideration as features of special concern, and

- Profile biological features and areas unique to the county for consideration in watershed coordination and countywide planning.

This study was conducted by the Montana Natural Heritage Program (MTNHP), which collects, maintains and provides statewide information on features of biological significance, including species of special concern and natural vegetation. MTNHP serves as a clearinghouse, assembling information from secondary sources such as museum specimens, published articles, and reports, and supplementing it with field studies.

\section{Terms}

This study focuses on plant "species of special concern," which are native species that are considered vulnerable to extirpation across their range or across the state due to inherent rarity, significant loss of habitat, or sensitivity to human-caused mortality or habitat disturbances. These are also referred to as "tracked" species, since information on their distribution and status is systematically compiled and continuously maintained by MTNHP. This study also addressed "watch species," which do not currently meet the criteria described above. These species may be in decline in Montana, or there may simply be too little information to accurately assess their status.

"Natural vegetation" refers to recurring associations of native plants ("plant associations") with characteristic composition and structure and relatively uniform environmental conditions. Natural vegetation provides habitat for native plants and animals, and includes the entire assemblage of interdependent species in a given environment. Specific forms of natural vegetation are referred to as "plant associations". In this report, we refer to plant associations by the names of one or more dominant plant species.

\section{Study Area}

\section{Background}

Sheridan County is an area of 1,720 square miles in the extreme northeast corner of Montana

(Figure 1). It was originally part of Big Horn County in 1864 , successively split off into smaller counties as part of Dawson County in 1889 , Valley County in 1893, and finally Sheridan County with Plentywood as the county seat in 1913 (Aasheim 1970). Big cattle company operations were in place before farming, and "Northeastern Montana was not merely the cowman's last frontier, it was his paradise" (Aasheim 1970).

When the Great Northern Railroad was constructed along the Missouri River in 1887 to connect Minneapolis to Helena, special transportation rates were offered to prospective settlers seeking quartersections of land to farm under the Homestead Act. A second major flurry of homesteading took place with the construction of a Great Northern branch line directly to Plentywood in 1910, about the time that an amendment to the original Homestead Act was made in 1909 to allow two quarter sections of land per homesteader for settlement. Barely 60 years later in 1971, over half of the total acreage in the county was dedicated to small grain production (Richardson and Hanson 1977). The county's greatest natural resource is its soil (Richardson and Hanson 1977) and an agricultural landscape prevails over much of Sheridan County today.

Against this backdrop of an agricultural landscape, we focused this study on three areas with the least extensive cropland. These areas coincide with some distinguishing landscape features that are described in the following text and represented in a map showing the topography of the Sheridan County area (Figure 2). 
Figure 1. Sheridan County study area
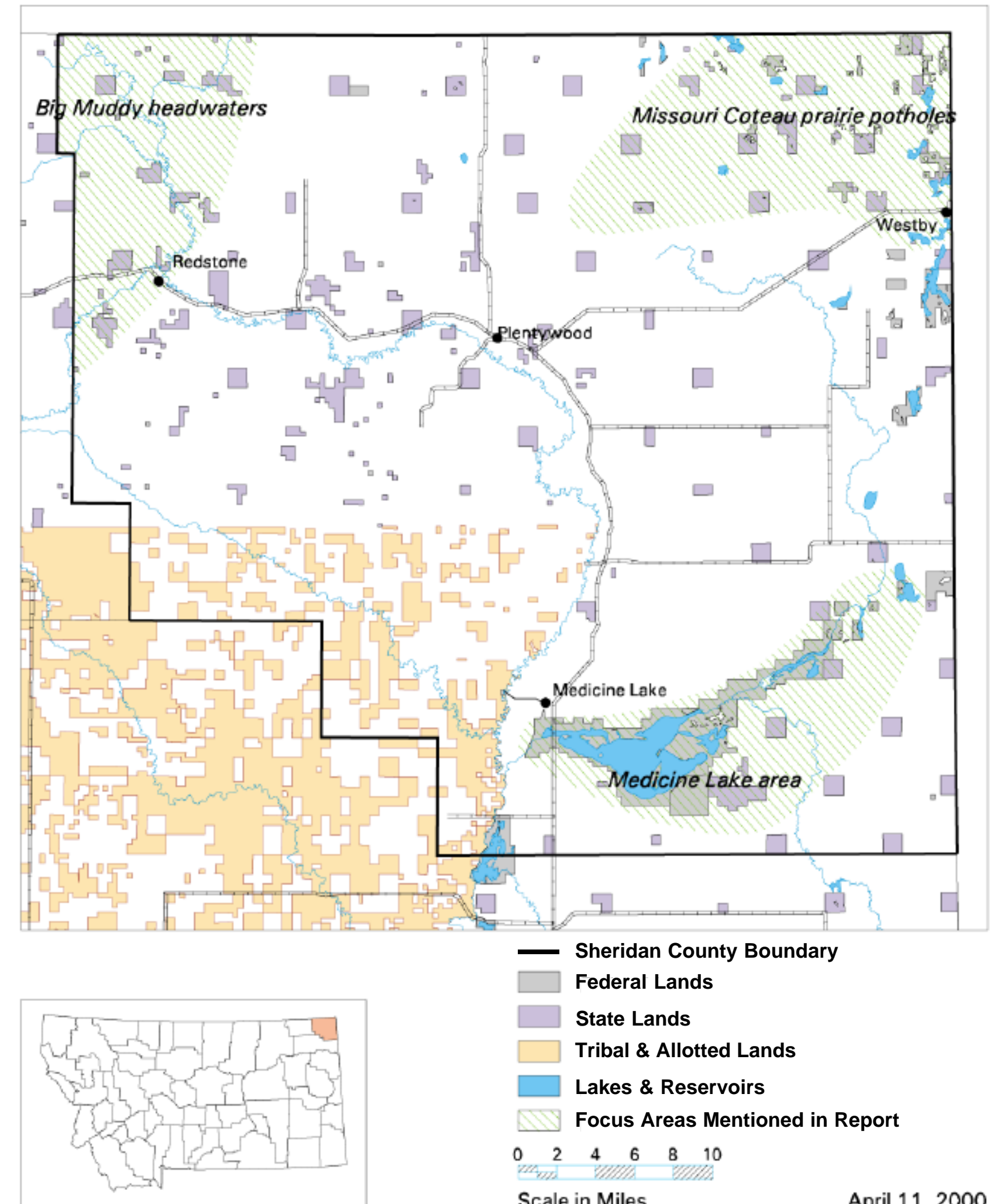

\section{Sheridan County Boundary}

Federal Lands

State Lands

Tribal \& Allotted Lands

Lakes \& Reservoirs

Focus Areas Mentioned in Report

$\begin{array}{llllll}0 & 2 & 4 & 6 & 8 & 10\end{array}$

Scale in Miles

April 11, 2000 


\section{Big M uddy headwaters}

Big Muddy Creek traverses Sheridan County, flowing southward into the Missouri River. The headwaters area includes segments that have continuous unplowed valleybottoms, slopes, and surrounding uplands. As the largest watercourse and valley in the county, it is a key landscape feature and provides connectivity for both wildlife and plants. The headwaters are located within the northwestern part of the county (Figure 1), and the headwaters extend into Saskatchewan.

\section{M edicine $L$ ake area}

Medicine Lake National Wildlife Refuge (NWR) contains the largest lake in the county. Wind has moved sand from the lakebed to create large sandplains to the southeast of Medicine Lake, bordered on the north and east by Sand Creek. They are some of the most extensive wind-borne sand deposits in eastern Montana (from Ross et al. 1955), with topography and substrate that distinguish them from the rest of the county. Lake Creek feeds into Medicine Lake, connecting it with extensive wet meadows and other wetlands upstream. It is located within the southeastern part of the county, including and east of Medicine Lake NWR (Figure 1).

\section{M issouri Coteau prairie potholes}

The Missouri Coteau is a rolling physiographic region that cuts a diagonal swath across the northeastern corner of Sheridan County (Figure 1). The Missouri Coteau Region generally has a high density of wetlands, with a tremendous range of size, depth, and alkalinity. These wetlands are collectively called "prairie potholes" in reference to the plains setting and rolling topography. This is the only part of Sheridan County without surface connection to the Big Muddy watershed, instead being made up of a series of small, closed-basin watersheds with limited development of watercourses.

\section{Climate}

Sheridan County has a continental climate with cold winters, warm summers, and peak rainfall early in the growing season. Rainfall patterns throughout the county are those typically conducive to agriculture, where precipitation is in excess of evapotranspiration (Figure 3 ). The growing season is usually110-125 days long, with roughly $80 \%$ of annual precipitation concentrated during that period. Annual precipitation ranges from 12-13 inches annually over the northwestern part of the county to 14-15 inches over the southeastern part. Steady, gentle rains usually occur in May, June and September, with the rest of growing season rainfall arriving in thundershowers (Richardson and Hanson 1977). It lies in the Eastern Glaciated Plains, 10-14" zone, as used to characterize state range sites (Ross and Hunter 1976).

As is typical of continental climates, precipitation varies greatly from year to year. For example, in Redstone, MT, one year in ten will have a total annual precipitation of less than 8.0 inches or more than 18.0 inches (1954-1970; Richardson and Hanson 1977). Similarly, Medicine Lake shows one in ten years with total annual precipitation of less than 9.5 inches or more than 19.1 inches (19411970; Richardson and Hanson 1977).

Average daily minimum temperatures in Redstone and Medicine Lake are at -5 degrees F in January and average daily maximum temperatures at 85 degrees F in July. However, even these figures mask the extremes - Medicine Lake holds the state record for the highest temperature on record at 117 degrees F. To the casual observer, daily temperature extremes may be the most noticeable.

Annual precipitation in 1998-99 when this work was conducted was close to the mean and generally favorable for the field study. In Redstone, the total for 1998 and 1999 was 16.62 in. and 12.66 in., respectively (NOAA). In Medicine Lake, the total for 1998 and 1999 was 14.68 and 13.4 in., respectively (NOAA).

\section{G eology and Soils}

The plains landscape of Sheridan County has a modest elevation range of 1,933-2,600 ft (Richardson and Hanson 1977) that belies a dramatic glacial history of repeated advances that forced the Missouri River out of the Hudson Bay watershed. The glacial deposits and topography dictate over soil characteristics, which are discussed in tandem for the three study areas. 


\section{Big M uddy headwaters}

One of the most pronounced landforms in the county is the Big Muddy Creek channel, a valley that is one to three miles wide, and originally $300 \mathrm{ft}$ deep in places, partially filled with outwash and alluvium (Figure 2; Witkind 1959). It contains the Big Muddy Creek, a perennial stream that is no more than 10 feet wide in most places, and represents the largest watercourse in the county. The north end of the Big Muddy valley and major tributaries make up the Big Muddy headwaters, where there are some of the largest areas of unplowed valley and adjoining uplands.

This broad valley, which is oversized for the stream now occupying it, is a major outwash channel formed near the margin of a glacial front over 12,000 years ago (Clayton et al. 1980). It has valleybottom deposits over 100 feet deep in places, including alluvial clay and outwash (Witkind 1959). The valley is bordered by terraces that are both pre-glacial and post-glacial in origin. In its northern reaches, it cuts through Fort Union Formation bedrock, variously made up of claystone, calcareous siltstone, and sandstone. Its headwaters and tributaries also cut into Flaxville gravel that top the Fort Union Formation in places.

Soils in the Big Muddy headwaters area are mainly well-drained silty clay loams and clay loams (Lambert-Zahill association) on the valley breaks and uplands. The valleybottoms are mainly welldrained silt loams and silty clay loams (HavrelonCherry association) on floodplains and fans (Richardson and Hanson 1977). The fine-textured soils and the steep topography along the valley signify distinctly more arid conditions despite modest differences in average precipitation in comparing this area with the following areas.

\section{M edicine $L$ ake area}

Another pronounced landform in the county is Medicine Lake, a large, shallow lake that fills an ancient valley. Medicine Lake, the adjoining sandhills, and contiguous valley of Lake Creek are centered along the other large glacial outwash channel in Sheridan County, the Dagmar outwash channel. This channel is broader than the Big Muddy Creek channel but nearly filled with outwash and alluvium (Figure 2). Part of its course may have been the pre-glacial valley of the Mis- souri River Valley (Witkind 1959). As a glacial meltwater channel, it was an arteriole of glacial run-off. Associated with Medicine Lake are large sand deposits that originate from the bed of the outwash channel. Most of the sand deposits are a thin veneer over till and bedrock. The dunes and gently rolling plains around them make up the Medicine Lake sandplains. In places, they have been reworked by the wind into small ridge systems parallel with the prevailing winds, forming choppy dunes.

Running northeast/southwest from Medicine Lake is Lake Creek, occupying what is thought to be a pre-glacial route of the Missouri River, now filled with outwash so that the wide valley is not very deep. Water levels were modified by a spillgate on the Lake, and a series of dikes above it that created shallow impoundments in the valley.

Most soils in the Medicine Lake area are welldrained to excessively drained soils on uplands, varying in sand or gravel content and drainage. They include the Blanchard association throughout the sandhills, composed of well-drained and droughty fine sands and loamy sands, and the Lihen-Parshall association throughout the gentle sandplains, composed of well-drained loamy fine sands and fine sandy loams. The broad Dagmar channel immediately northeast (upstream) from Medicine Lake has the contrasting McKenzie association, composed of poorly drained silty clay loams across the lowlands.

\section{M issouri Coteau prairie potholes}

The outwash terrain around Westby and the collapsed moraine landscape around Comertown make up the Missouri Coteau prairie potholes area, with its high density and diversity of wetlands. The associated topography does not show up at the scale of the topographic map in Figure 2, except for the largest alkali lakes. But it represents the most complex and irregular topography in the county. This area experienced at least three episodes of glacial advances, indicated by maximum icemargin positions (Figure 4). The most recent entered the northeastern corner of the county, and left hummocky collapsed glacial sediment fringed by outwash channels. 
Figure 2. Topography of Sheridan County area

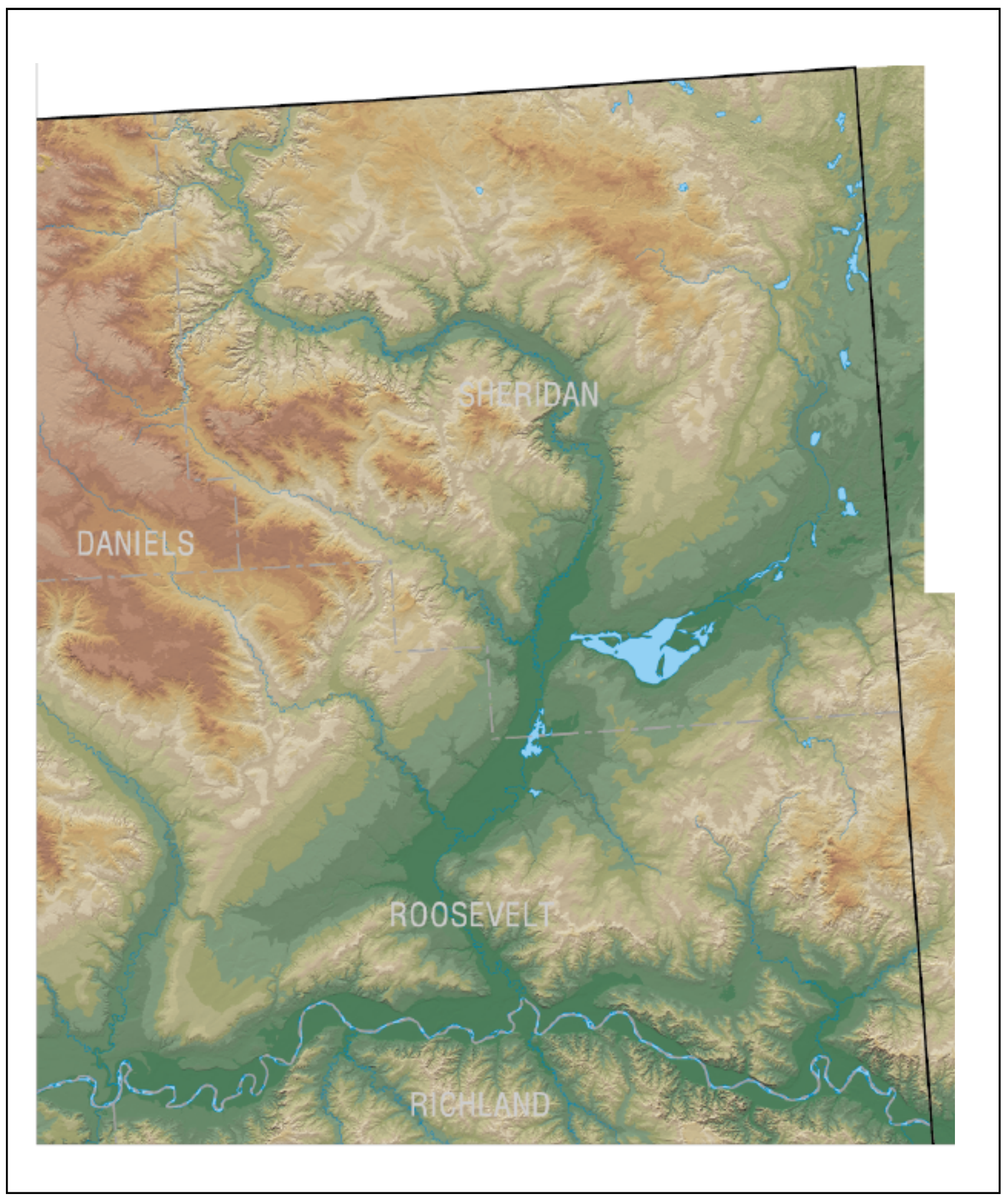


Figure 3. Climate of Plentywood, MT. Diagram showing average monthly temperature and precipitation. Growing season length is the number of frost-free days, i.e., with mean daily minimum above 0 degrees $\mathrm{C}$.

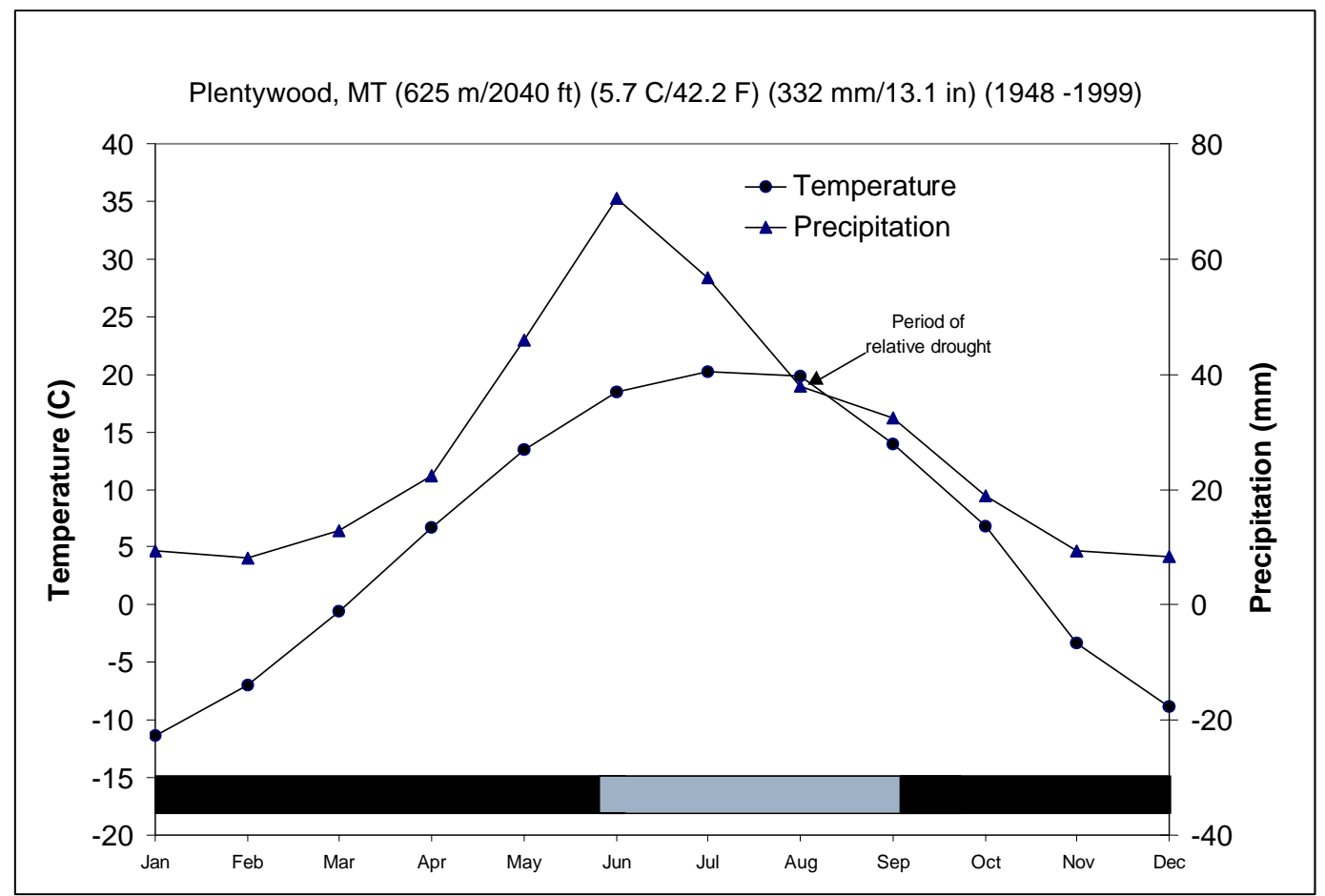

Figure 4. Maximum extent of continental ice margins in the Great Plains

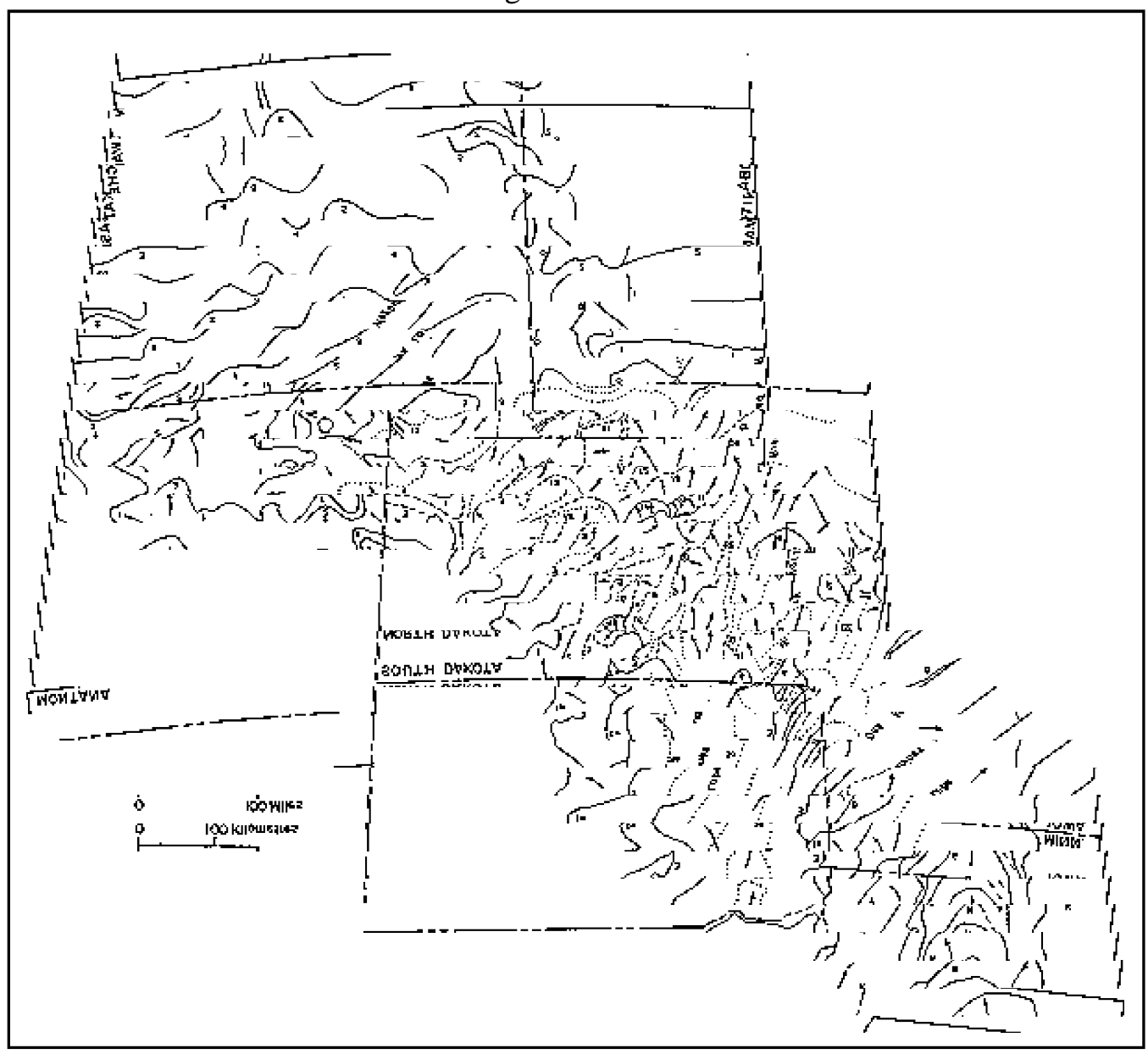


Hummocky topography is thought to result from lateral slumping and sliding of the sediment that overlies the glacier as it melts and collapses in place (from Clayton et al. 1980), sometimes referred to as "dead-ice moraine" (Kantrud et al. 1989). Steep hummocky terrain is a characteristic feature in the Comertown area, producing wetland basins of all sizes and shapes and high wetland densities. Glacial outwash was deposited on stagnant ice, and the overlying sand and gravel slumped to form irregularly hilly terrain with many wetlands, often including large, shallow, saline lakes. Outwash channels around Westby are comprised of bedded glacial sediments that lie in topographic low points in closed-basin watersheds. They form some of the most extensive alkali lake systems in the state and act like large evaporation ponds where salts accumulate.

A great variety of soil textures are reflected in these glacial deposits. Tills in moraines are generally silty and clayey materials that are not very permeable, while outwash deposits also include sand and gravel layers (Kantrud et al. 1989). Soils over much of the moraine deposits are mapped as ZahillWilliams-Dimmick association. Clay loams and loams prevail in the first two series (slopes 15$45 \%$ ). The Dimmick association is made up of poorly-drained silty clays in wetland basins.

\section{Wildlife}

The most popular game animals of the county include white-tailed deer, waterfowl, grouse, partridge, and pheasant, as highlighted in the county soil survey (Richardson and Hanson 1977). The full array of wildlife resources of Sheridan County are best-documented in Medicine Lake NWR. The refuge was established in 1934 in recognition of its outstanding waterfowl nesting habitat for Canada geese and ducks. It has since been recognized for its value for colonial nesting birds, for migratory waterfowl, and as habitat for upland grassland birds, including passerines and upland game birds. It has been the center of activity for most wildlife studies in Sheridan County and adjoining areas.

In addition, there are animal species of special concern or watch species known from breeding records in the county (Appendix A), including species of birds, reptiles, an amphibian, and insects. Most of them are birds, and most of these occupy wetlands or lakes. The ones that are dependent on open water are restricted to Medicine Lake NWR. Only the Piping Plover (Charadrius melodus) is federally listed as threatened. Sheridan County has the largest numbers of Piping Plovers in Montana, and a relative abundance of grassland birds, some of which are in decline elsewhere over much of their range.

\section{M ethods}

\section{O verview}

The purpose of this systematic inventory was to identify those native plant species and plant associations that are the least common in the state or throughout their range, as well as high quality examples of more common plant associations. We began by compiling and reviewing available data in the county and adjacent counties, states, and provinces. We then conducted targeted field surveys to fill information gaps for plant species and plant association types.

Field surveys for plants and plant associations were conducted in the summers of 1998 and 1999. The first year was aimed at developing a list of plant species of special concern and starting to document the plant associations that were known or suspected from the county. We also evaluated the distribution and rarity of species and plant associations, to prioritize them for further survey work.

The second year was aimed at more thoroughly documenting priority species and plant associations, focusing on areas of relatively intact vegetation. Areas of relatively unbroken landscape and localized vegetation features were identified using color infrared aerial photos (NWI; 1:65,000; taken in 1983). We transcribed notes onto U.S.G.S. topographic maps (7.5') that were carried in the field. In the case of some areas with complex patterns, copies of black-and-white aerial photos $(8 "=1$ mile) were ordered from the USDA Farm Service Agency for use in fieldwork. The soils information in the county soils survey (Richardson and Hanson 1977) was also used for reference in 
the field. We obtained a great deal of valuable information by talking with people familiar with the county's species and landscapes.

Our principle floristic references were the Great Plains Flora Association (1977, 1986), Dorn (1984), and Hitchcock and Cronquist (1973). Looman (1982) was consulted to the extent that vegetative grass identification was needed. Nomenclature used in this report is generally consistent with these references, except for revisionary taxonomic treatments, mainly for grasses (Kartesz 1994). This means incorporating unfamiliarsounding names for some dominant and indicator species, particularly the wheatgrasses (Agropyron and Elymus in the traditional sense). Thus, western wheatgrass, called Agropyron smithii in Booth (1950) and Elymus smithii in Dorn (1984), is referred here as Pascopyrum smithii. Synonymous names for the plant species of concern are crossreferenced with the information on each species. Synonymous names of dominant and indicator plants are cross-referenced in Appendix B. We have also cross-referenced each species by both scientific name and common name the first time the species is mentioned under each heading. Common names are based mainly on the list developed by the U.S. Forest Service of Region 1, generally consistent with major floras.

Records for all populations of plant species of special concern and for the best examples of plant associations were transcribed from field forms onto standardized forms, geo-referenced, and incorporated into the Heritage databases as representing noteworthy occurrences of biological diversity.

\section{Vegetation ecology methods}

The first year of vegetation survey took into account the range of environmental conditions; our survey spanned the diversity of "habitat extremes" in order to chronicle the range of natural vegetation in the county. In addition to photointerpretation results, we were aided by experts who identified unusual or specific habitats worthy of investigation. These features were concentrated in the Medicine Lake area, Missouri Coteau prairie potholes area, and the Upper Big Muddy area.
First year survey results were compared against our list of known Montana plant associations to identify new discoveries or uncommon plant associations. Based on the findings, we designed the second year of vegetation sampling to characterize:

- Well-developed examples of common plant associations in outstanding condition;

- Plant associations of potential statewide or rangewide rarity and significance;

- Provisional new plant associations or ones not previously known from northeastern Montana.

Plant associations were documented in terms of their plant species composition, structure and associated abiotic environmental conditions in 117 ecology plots (Figure 5), eight of which were previously sampled at Medicine Lake NWR (Cooper and Heidel 1999). We included three plots outside the county but within a mile's distance and useful in describing county features. To document species composition, we sampled vegetation in the representative $1 / 10$ acre plots $(37.2 \mathrm{ft}$ radius or equivalent). Ecology plots were placed to represent homogeneous natural vegetation and uniform environmental conditions across the plot, and the least disturbance. Canopy cover values for all species present, plot location and environment characteristics were recorded on a survey form that was later computerized for analysis and permanent data storage. After each plot was subjectively assigned to a plant association, average plant cover and constancy by plant association type was calculated using Eco analysis (V.9.12.2) computer software. The results were used to describe plant associations of Sheridan County.

Vegetation sampling focused on herbaceous grassland plant associations at the outset because grasslands are the prevailing vegetation of the county, representing well over $90 \%$ of the existing natural vegetation. After the first year, it also became apparent that they are among the distinguishing vegetation features as well. Also after the first year, we compared wetland observations with wetland classification information in Montana (e.g., Hansen et al. 1995). It became apparent that there were many wetland plant associations present in the county that have not been documented in the 
Figure 5. Vegetation study areas in Sheridan County

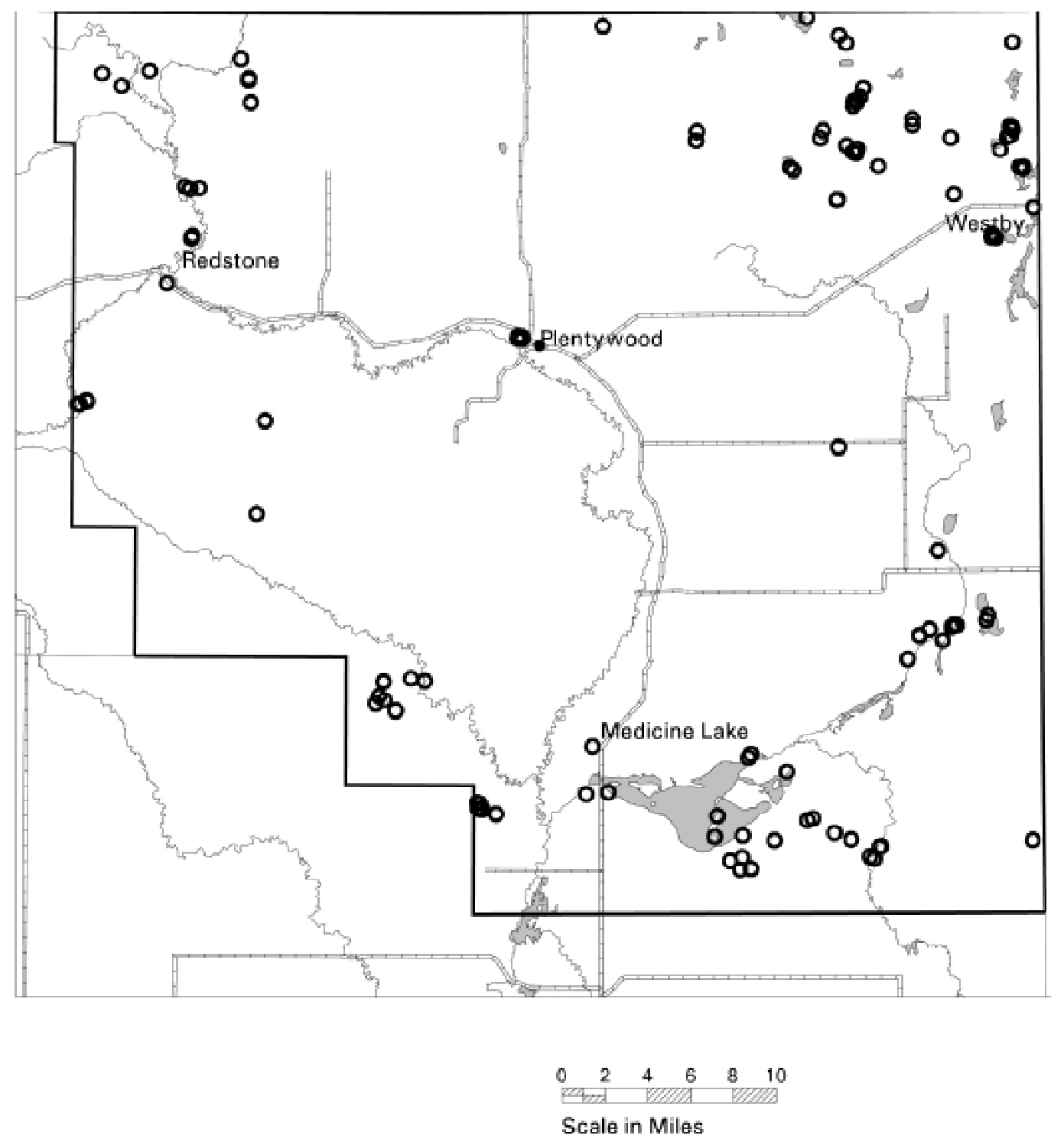

state. Wetlands are also distinguishing ecological features of the county. We used the wetland vegetation work conducted elsewhere in the Missouri Coteau region by the Northern Prairie Biological Research Station (Stewart and Kantrud 1971, 1972, and Kantrud et al. 1989) to start documenting wetland plant associations that are "new" to Montana.

Vegetation classification followed the National Vegetation Classification System (NVCS;

(Grossman et al. 1998), a system that:

- Is defined in terms of recurring vegetation attributes;
- Emphasizes natural existing conditions;

- Uses a classification hierarchy based on structure and floristic composition;

- Can identify vegetation units based on both quantitative and in some cases qualitative data.

The NCVS classification is hierarchical and combines floristics at the lowest levels (associations and alliances) and structure (physiognomy) and overarching climate conditions at the highest levels. The plant association is defined in terms of the dominant species of the uppermost vegetation layer, and any co-dominant species, diagnostic species, or the dominant species of shorter vegetation layers. Literature review and stand data 
analysis had been conducted in Montana to produce an initial working list of the plant associations in the state.

Prior to this study, the natural vegetation of Sheridan County and northeastern Montana was represented by scattered references. Range sites of Sheridan County are described in the soil survey (Richardson and Hanson 1977) and provide a forage-production perspective on the most common grassland vegetation. More detailed vegetation descriptions were available for the Medicine Lake Sandhills, and to a lesser degree in the Comertown Prairie (Lesica 1987a, 1987b, respectively). In 1990, vegetation plots were sampled near but not within Sheridan County in a vegetation study of northeastern Montana (DeVelice et al. 1995). In 1997, vegetation was sampled at several sites in Medicine Lake National Wildlife Refuge (NWR) in a study of Research Natural Areas that included vegetation ecology and botany (Cooper and Heidel 1999). Apart from these sources, most pertinent vegetation information was from studies in adjoining counties, states and provinces and from the recently compiled state and regional NVCS classifications representing Great Plains states (Reid et al. 1999, Faber-Langendoen 1999). We have drawn on those references to provide background information, to help build a broad picture of vegetation diversity in the county, and to refine the working list of plant associations in the state. The literature that was reviewed to describe each of the Sheridan County plant associations in the appendix is incorporated in the Literature Cited section at the end of the report.

Such a classification approach offers a refinement of the sweeping generalizations that have commonly been used in characterizing Great Plains vegetation as indicated by grass height (tallgrass, mixed-grass, short-grass). Even though environmental modifiers have been used with this system to characterize "ecological sites," or "natural communities" (e.g., Heidel 1992), such an approach has only limited consistency within state boundaries, and quickly loses cohesion over the expanse of North American mid-continental grasslands as the most extensive formation on the continent.

\section{Botany methods}

Prior to conducting fieldwork, we prepared a "search list" of species and their habitats to use as a reference in planning and conducting field surveys (Appendix C). It represented those Montana plant species of special concern previously documented from the county or from adjoining counties, consistent with the most current species of Montana special concern list (Heidel 1999). It also included native plant species not known from Montana but occurring in nearby areas of North Dakota and Saskatchewan that may have suitable habitat in Montana. Information on the phenology, habitat needs, and known distribution of these target plant species was assembled from Natural Heritage Program databases, published and unpublished literature, and species checklists from adjoining states and provinces.

Aerial photographs, USGS topographic maps, geologic maps, and soil survey maps were consulted to identify areas of potential habitat for ground survey. We targeted previous records of plant species for re-visit if there were questions on the species' location, setting, or population numbers. Botany fieldwork was conducted mainly in the second year of study, often in tandem with vegetation ecology work.

During fieldwork, data were collected on the biology, habitat, population size, and any indications of trend for the target species. Locations of target species were mapped onto U.S.G.S. topographic maps (7.5'). Information gathered in the field was entered into the MTNHP data system, and mapped locations were digitized if the population extended beyond a single place on the landscape. Voucher specimens were collected if population numbers were sufficiently large to avoid impact (Montana Native Plant Society 1993).

Previous collection records of the plant species of special concern in Sheridan County have already been compiled in the MTNHP database from the major herbaria of the state and most major herbaria of the region, as well as from the literature. For other questions of species distribution, as in the case of watch species, we used two references with county dot maps (Booth 1966, Great Plains Flora Association 1977), the general descriptions of state distribution that are in Dorn (1984), and prelimi- 
nary checklists in unpublished reports. We also reviewed a set of plant specimens collected on Medicine Lake NWR for any collections of plant species of special concern, and as habitat indicators. We maintained a running list of the vascular flora over the course of the field surveys, and collected species that could not be positively identified in the field for later determination. Specimens will be deposited at the University of Montana (MONTU).

We reviewed current plant taxonomy literature and other sources of information in making determinations and assembling the most current status information on each of the species and evaluating it in light of study results. The literature review for each of the plant species that are described in the appendix is incorporated in the Literature Cited Section at the end of this report.

\section{Results}

\section{O verview}

This study identified 42 plant associations and 12 plant species of special concern plus three watch species in Sheridan County. Five of the plant associations are newly-described types, and nine more are new to the state. They include highly productive grasslands, wet meadows, and sandhill plant associations. These are particularly significant as the only and best of known examples within Montana, and may be of significance in the United States or the Great Plains as a whole.

Eight of the fifteen plant species of special concern were documented in Sheridan County for the first time. Results significantly expand their known state distribution. Only Sisyrinchium septentrionale (northern blue-eyed grass) is known in Montana only from Sheridan County. Three of the 15 species were not successfully relocated but survey work was not adequate for saying that they are no longer present.

Results of the vegetation ecology and botany work are highlighted in this section. They are detailed in tables and text that are in the appendix with descriptions of each plant associations and plant species identified in this study. The descriptions include a summary of their distinguishing characteristics, habitat, status, state and county distribution, and management considerations.

The eastern end of the county supports the greatest diversity of native vegetation types and significant plant species, in part because it has the most extensive and diverse wetland habitats. These are concentrated in the Medicine Lakes area and the Missouri Coteau prairie potholes region. Another diverse area with good ecological diversity and a number of significant species is the Big Muddy Creek headwaters. The biological diversity and significance of these three study areas is summarized below.

\section{M edicine L ake area}

The Medicine Lake area has the most extensive open water habitat in the county. Medicine Lake is the largest lake in the county, and Brush Lake is the deepest lake in the county, both lying along the Dagmar outwash channel. Medicine Lake is the defining feature of Medicine Lake NWR, established in 1934 for its high value in providing nesting habitat for waterfowl. Water bodies near Medicine Lake and Brush Lake support Piping Plovers (Charadrius melodus), a threatened shorebird. Brush Lake has also provided insight in to the ecological history of the area through research on its uninterrupted sediment records (e.g., Umbanhowar 1996).

The Medicine Lake NWR has already been documented as harboring seven bird species of special concern that require open water, some of them occurring nowhere else in the county. They include American White Pelican (Pelecanus erythrorhynchos), Black Tern (Chlidonias niger), Black-crowned Night-heron (Nycticorax nycticorax), Caspian Tern (Sterna caspia), Forester's Tern (Sterna forsteri), Franklin's Gull (Larus pipixcan), and Great Blue Heron (Ardea herodias). Altogether, this area supports at least 15 Montana animal species of special concern, including the Ottoe Skipper (Hesperia ottoe), a butterfly which usually feeds on the nectar of purple coneflower (Echinacea angustifolia). 
Figure 6. Photographs of the Medicine Lake area



The Medicine Lake sandhills are among the largest sand deposits in eastern Montana, with intact uplands made up of the needle-and-thread - lemon scurfpea plant association.

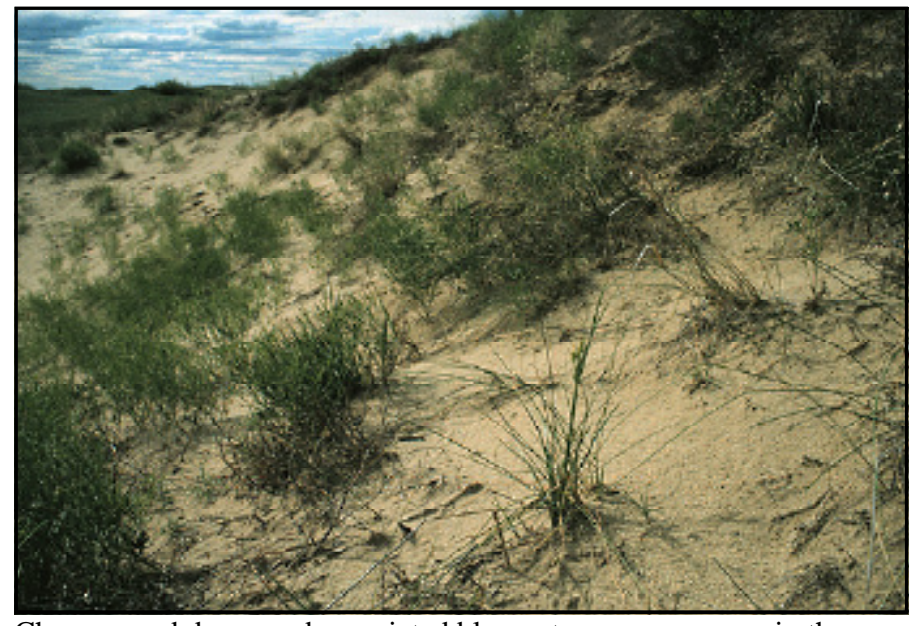

Choppy sand dunes and associated blowouts are uncommon in the sandhills, and three of the plant species of special concern are concentrated in these small, scattered areas, including Schweinitz' flatsedge (lower right).

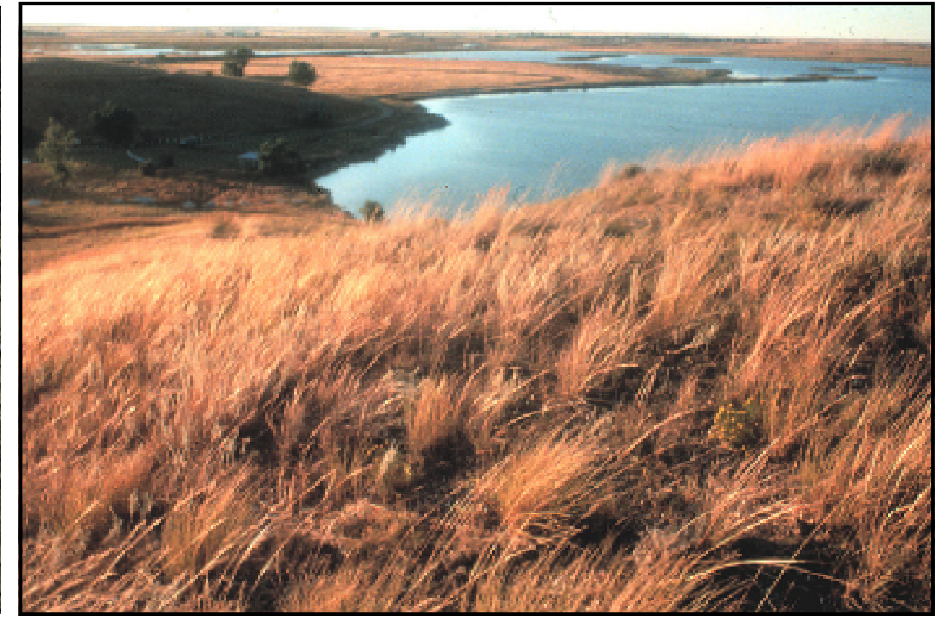

Medicine Lake is the largest lake in the county, with major waterfowl and shorebird values, and use by seven bird species of special concern that require open water.

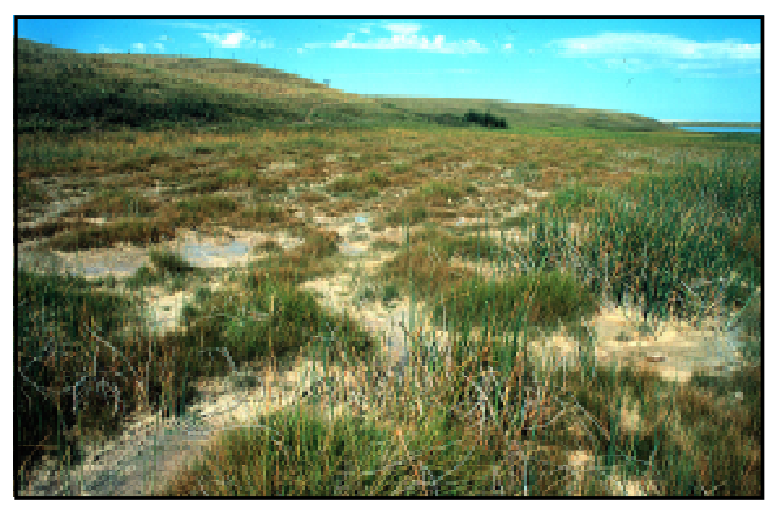

Peatland communities made up of parallel bands of "chalky" marl pools and mounds are uncommon along outwash channels, including Lake Creek. 
Figure 7. Photographs of the Missouri Coteau prairie pothole area
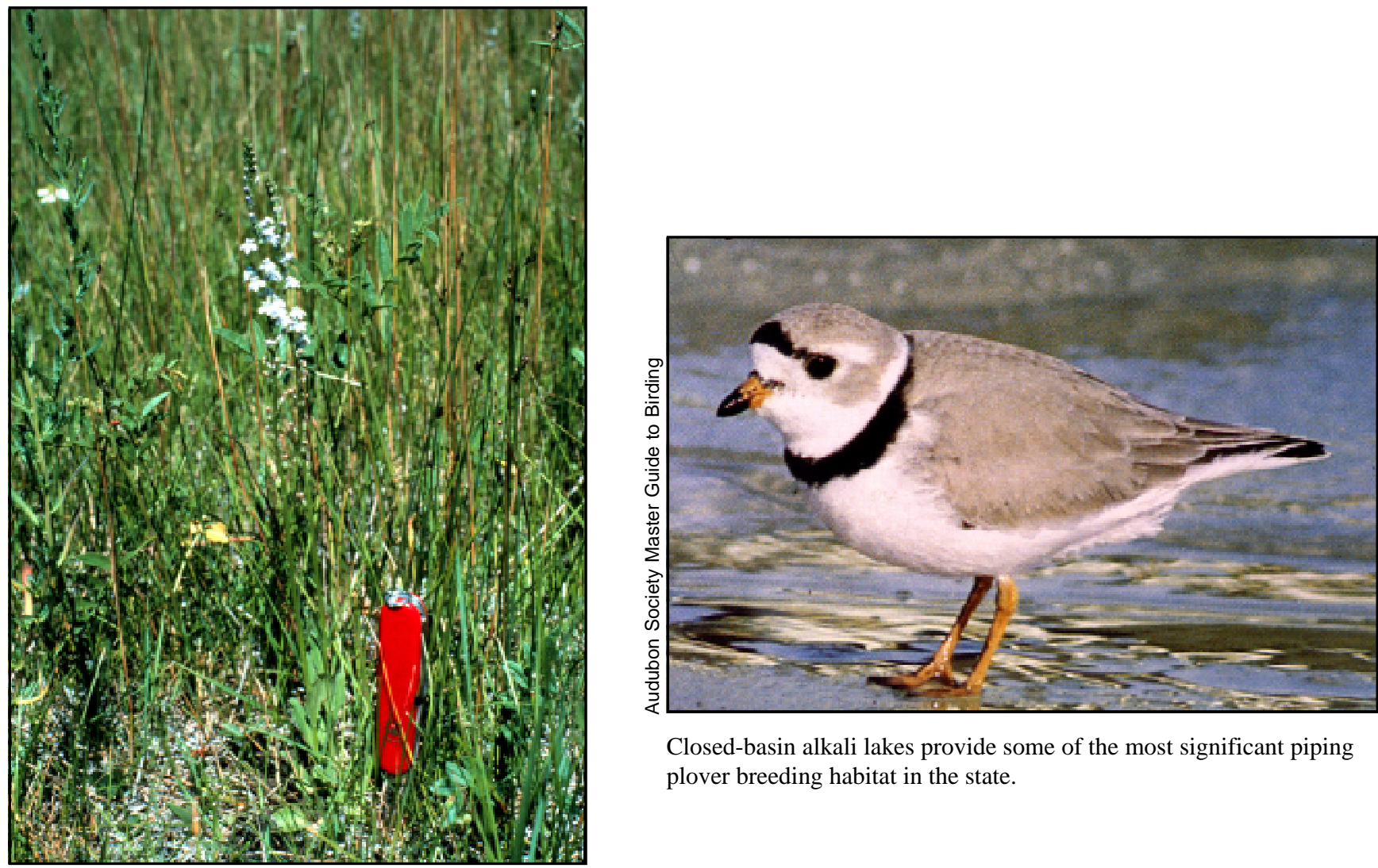

Closed-basin alkali lakes provide some of the most significant piping plover breeding habitat in the state.

Pale-spike lobelia is among four Montana plant species of special concern that are in prairie pothole landscapes and restricted to wetland margins.

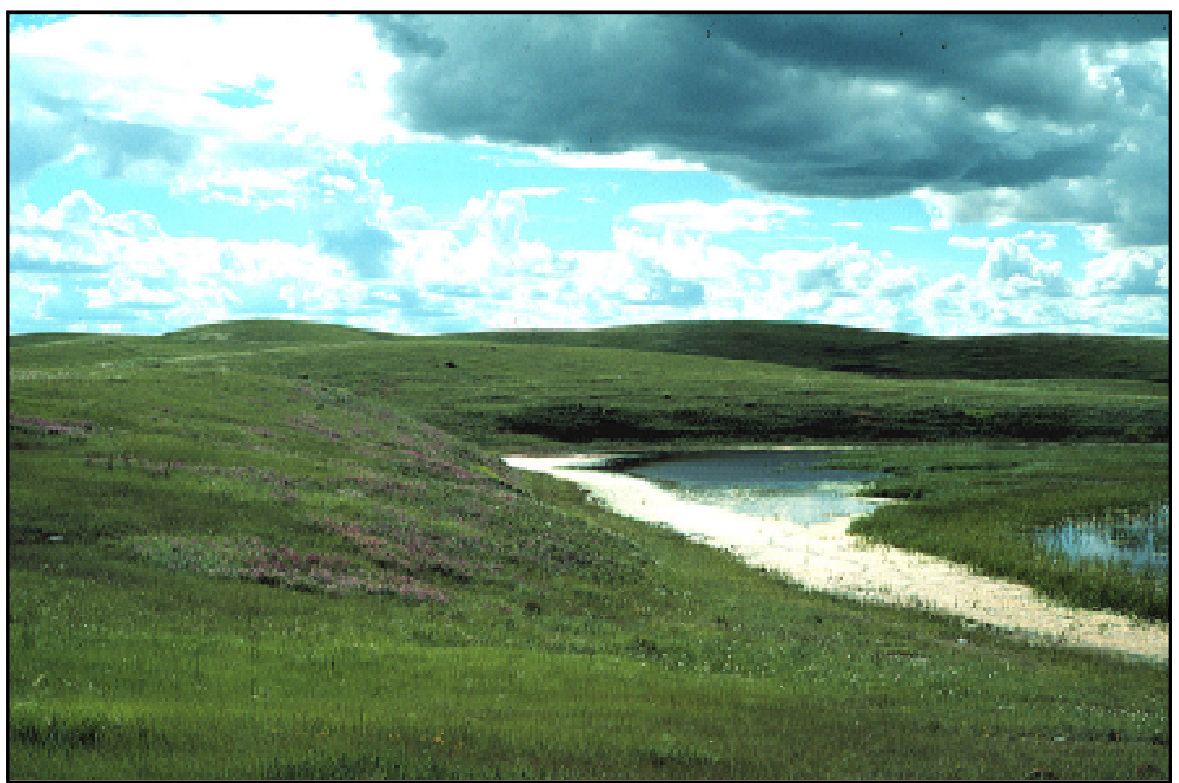

The rolling prairie pothole topography encompasses a mosaic of grassland and wetland plant associations, including the northern porcupine grass plant association (left of wetland), with its rich forb and shrub component that turns north-facing hillsides into swaths of earlysummer color. 
Figure 8. Photographs of the Big Muddy headwaters area

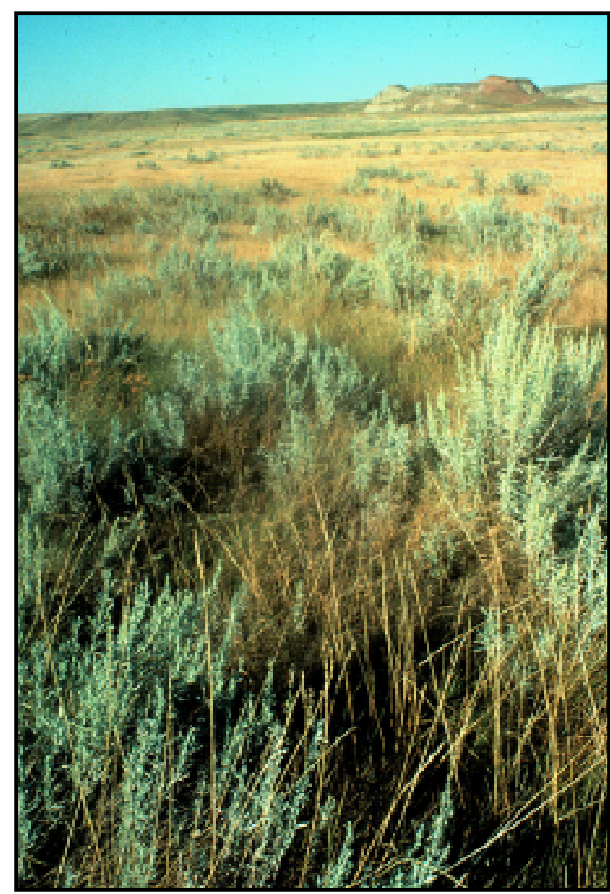

Intact valley bottom segments and bordering tablelands distinguish the Big Muddy headwaters area.

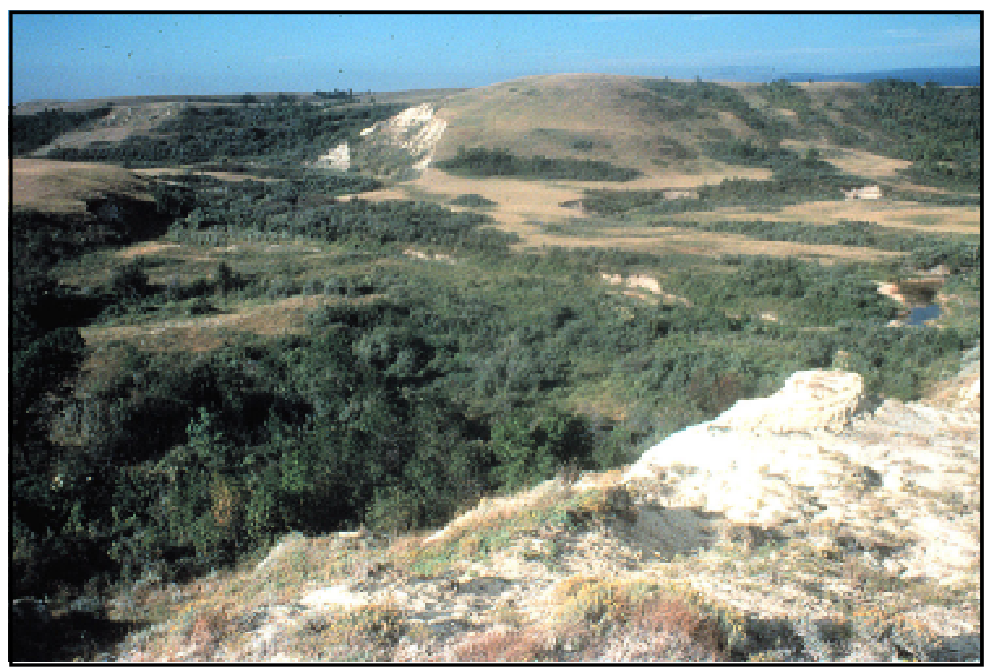

Valley segments with intact spring-fed wetlands and woodlands are even less common than the typically dry, open valley settings along the Big Muddy and its tributaries.
Also present are remnants of some of the more extensive wet meadows in the county in widelyscattered pockets. One of the wet meadow types, the Panicum virgatum - Muhlenbergia richardsonis - (Schizachyrium scoparium) plant association, is newly-described. This once-widespread community is reduced to small tracts. Small, isolated pockets of peatland that were never extensive are also found in the area.

The nearby sandplains are among the largest windborne sand deposits in eastern Montana judging by the geologic map of the state (Ross et al. 1955). They harbor five plant species of special concern, at least three of which have their highest known numbers for the state in this area where they are scattered across the landscape, including Schweinitz' flatsedge (Cyperus schweinitzii), Fendler cat's-eye (Cryptantha fendleri), and plains phlox (Phlox andicola). One of the sandhill vegetation types, the Stipa comata - Psoralidium lanceolatum plant association, is described here for the first time, corroborating reports in earlier work by Lesica (1987a). A composite view of distinguishing Medicine Lake area features is presented in Figure 6.

\section{M issouri C oteau prairie potholes}

The rolling Missouri Coteau prairie pothole terrain contains the highest diversity of plant associations, highest densities of wetlands, and some of the largest unbroken prairie landscapes in the county (Figure 6). This rich mosaic of grassland and wetland associations is part of the North American Prairie Pothole Region, a region that supports 50\% of continent's annual duck production in an average year though it covers an area that is only $10 \%$ of the net waterfowl breeding area (Smith et al. 1964; in Kantrud et al. 1989).

Five Montana wetland plant species of special concern have been found here. Four occupy the dynamic, vulnerable wetland perimeter, including many-headed sedge (Carex sychnocephala), chaffweed (Centunculus minimus), pale-spike lobelia (Lobelia spicata) and northern blue-eyed grass (Sisyrinchium septentrionale). Three of the grassland plant associations are "newly-described," based on vegetation sampling data, review, and synthesis of the literature; also corroborating a report of the Elymus lanceolatus - Stipa comata plant association by Lesica (1987b). All nine of the wetland plant associations that are new to Montana 
are in this area, and most of them were not found outside of it.

Closed-basin alkali lakes are best-developed and most extensive in this area, with specialized stresstolerant plant associations and some of the most significant Piping Plover (Charadrius melodus) breeding habitat in the state. Other wetland bird species of special concern are also known from this habitat, or the springs and seeps feeding into them. In our sampling, the alkali lake systems had the highest concentration of plant associations that did not correspond with any of those documented in existing vegetation classification literature, including both upland and wetland associations dominated by species of wheatgrass. A composite view of distinguishing Missouri Coteau prairie pothole features is presented in Figure 7.

\section{Big M uddy headwaters}

The Big Muddy headwaters bring together species of the Canadian Parklands, Great Plains, and the American Southwest, in its intact bottomlands and adjoining tablelands. It includes areas of welldeveloped Badlands and spring-fed tributaries. The results are a biogeographic melting pot of sorts, although there are few species of special concern here. Two woodland plant species that are on the state watch list, lavender hyssop (Agastache foeniculaceum) and common agrimony (Agrimonia gryposepala), were found in or near this area for the first time in the county in the course of the study.

The Big Muddy Creek in and below the headwaters area is a low-gradient perennial stream. It traverses the county in an open-water corridor, providing a landscape link between the Medicine Lake area and the Big Muddy headwaters. It supports a productive submergent plant association that includes sago pondweed (Potamogeton pectinatus), common watermilfoil (Myriophyllum spicatum), and horned pondweed (Zannichellia palustris). A composite view of distinguishing Big Muddy headwaters features is presented in Figure 8.

\section{0 ther areas}

Below the headwaters, a number of tributary valleys and side coulees along the west side of the Big Muddy valley support luxuriant spring-fed coulees, intact but dry bottoms in outstanding condition, and cool, shady ravine woodlands. Even higher in the uplands, escarpments rise to the southwest, and their most sheltered slopes contain the largest woodlands in the County, outlying habitats that greatly contribute to plant and animal diversity. In places, the Green Ash Woodland gives way to sheltered pockets of Aspen Woodland.

\section{Vegetation E cology R esults}

We identified a total of forty-two plant associations in Sheridan County through field survey. This includes five that are described and documented as plant associations for the first time, and nine that were not previously known from Montana, though they had been previously described elsewhere (Appendix D, Appendix E). The large number of plant associations identified as new to Montana point to the paucity of vegetation information that has been available for eastern Montana in general and the Missouri Coteau in particular.

The five newly-documented plant associations are all grassland types. Three of these are dominated by species not widely recognized as forming discrete plant alliances in the United States, including thick-spike wheatgrass (Elymus lanceolatus) and northern porcupinegrass (Stipa curtiseta). These are northern species of wheatgrass and needlegrass that are more common in Canada than they are in the U.S., and are not poorly-represented in the vegetation ecology literature of the United States. Major Canadian references on vegetation have not been completely integrated into plant association classification, and for this study, we have simply confirmed the presence or variants of these types in Montana, cross-referencing them to the Canadian literature. The three "newly-documented" plant associations of the northern plains include:

- Elymus lanceolatus - Stipa comata Herbaceous Vegetation

- Elymus lanceolatus - (Koeleria macrantha) Herbaceous Vegetation

- Stipa curtiseta Herbaceous Vegetation

The other newly-described associations are more characteristic of the plains to the east: 
- Panicum virgatum-Muhlenbergia richardsonis - (Schizachyrium scoparium) Herbaceous Vegetation

- Stipa comata-Psoralidium lanceolatum Herbaceous Vegetation

A series of schematic landscape cross-sections shows the placement of the most characteristic grassland plant associations in the three study areas (Appendix F). We have also developed a preliminary cross-reference between the range sites and the plant associations to help relate our results to soil survey data and range management literature (Appendix G). These were created after fieldwork when it became apparent that so many of the plant associations were not recognized in existing compiled classifications.

Most of the plant associations identified as new to Montana are wetland types. Wetland classification work conducted in the Missouri Coteau outside Montana generally applies in Sheridan County (e.g., Kantrud et al. 1989, Stewart and Kantrud 1972), which characterizes the vegetation for all of the common prairie pothole wetland settings in Sheridan County. One of the more unusual and intrinsically rare wetlands is the few-flowered spike-rush (Eleocharis quinqueflora) marl fen, a patterned rich fen representing a form of alkaline peatland, otherwise only reported from North and South Dakota. It occurred not only in the "stringand-flark" peatland patterning that has been reported for related peatlands in European literature, but in bands formed in concentric circles around springheads in patterns suggesting domebuilding accretion and collapse.

We ran vegetation analysis on 117 vegetation sampling plots (including 8 plots from earlier work). Of the 57 plant associations, only 11 are documented by four or more vegetation sampling plots, including most of the types which are newlydescribed or new to Montana. The sample size for our characterizations is limited.

Appendix E includes descriptions of all the plant associations we identified. Those described in greatest detail are newly-documented types, types documented for the first time in Montana, or types occurring extensively in the county. They are organized into the groups presented in Table 2, with the exception that wetland types have been grouped separately from grassland types rather than combined under "herbaceous vegetation." We present grassland types first as the predominant vegetation of the county.

Fifteen of the fifty-seven plant associations that we sampled in this study were noted from no more than two plots or observations, and they do not correspond to plant associations described for immediately adjoining areas of the Great Plains. Most of these are localized features, and many are associated with alkali lake systems. They warrant expanded field investigation and further consultation with vegetation ecologists in Saskatchewan, Alberta, and North Dakota. They are listed at the end of Table 2 as undescribed types, and treated at the end of the grassland, wetland, shrubland, and woodland sets in Appendix E.

\section{Botany Results}

This study identified twelve Montana plant species of special concern documented for the county and three that are on the state watch list (Appendix H, Appendix I). Seven of the species are restricted to wetlands of the Missouri Coteau and Medicine Lake areas, and five are restricted to sandhills of the Medicine Lake area (Figure 9). They reflect requirements for some of the habitats that are particularly well-developed in the county, namely wetlands and sandplains. It is important to note that most of the species are at the western margins of their distribution and are widespread elsewhere. Some may be vulnerable to rangewide decline. Most of them are known from few places in the county, though they may be locally common at those sites. These species are key parts of county biological diversity.

About half of these fifteen species were documented for the first time in Sheridan County in the course of this study, though none are new to the state flora.

The five plant species of special concern that are newly documented in the county include:

- Chaffweed (Centunculus minimus)

- Mealy primrose (Primula incana)

- Ovalleaf milkweed (Asclepias ovalifolia)

- Silky prairieclover (Dalea villosa var. villosa)

- Smooth goosefoot (Chenopodium subglabrum) 
Figure 9. Plant species of special concern in Sheridan County

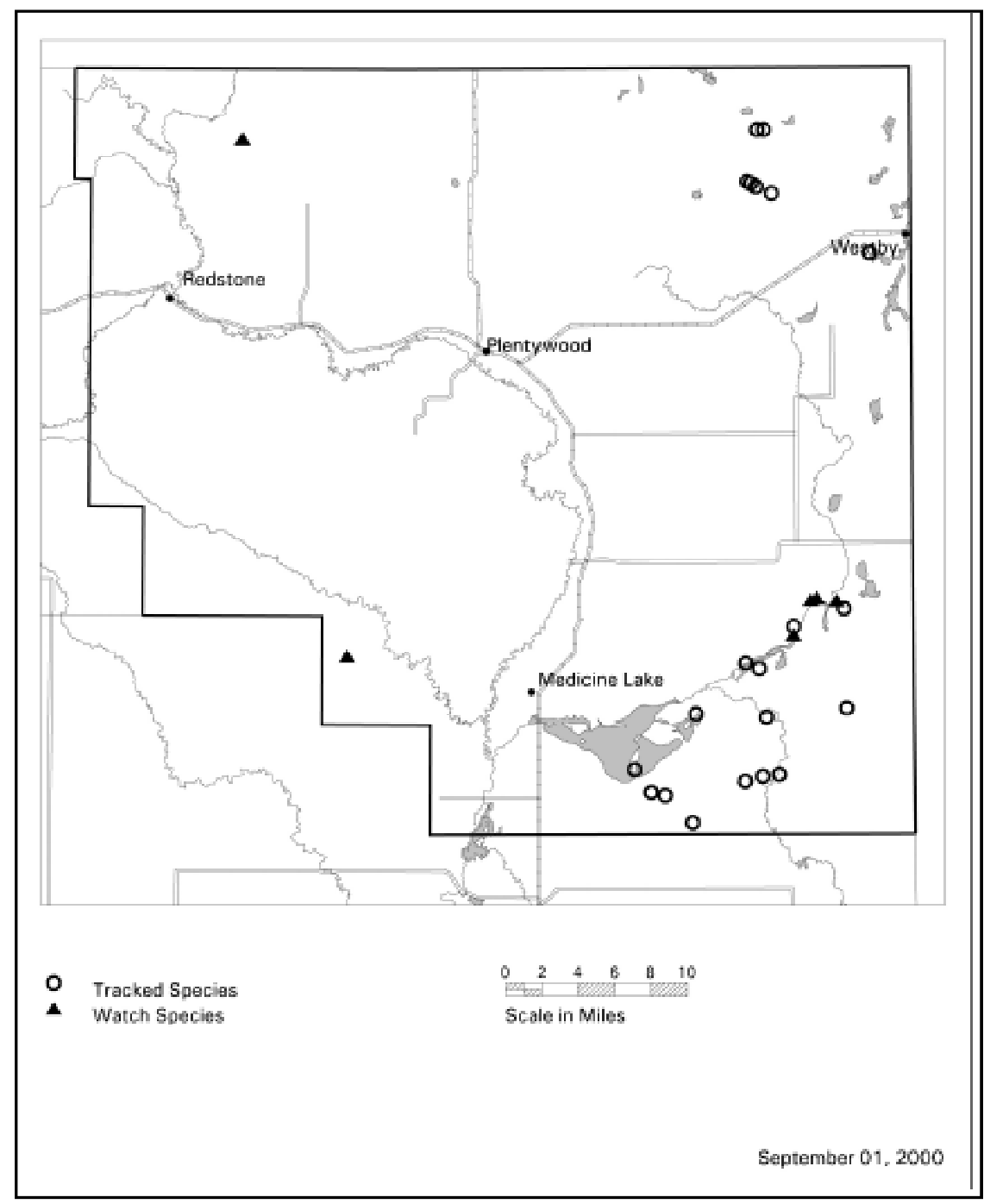

The three watch list plants species newly documented in the county are:

- Common agrimony (Agrimonia gryposepala)

- Kalm's lobelia (Lobelia kalmii)

- Lavender hyssop (Agastache foeniculaceum)

Hairy four o' clock (Mirabilis hirsuta), was recently dropped from the Montana plant species of special concern list because we found it in a broad range of natural and disturbed habitats, occurring repeatedly in the Medicine Lake area (Cooper and Heidel 1999). It is possible that some species like plains phlox (Phlox andicola) are much more widespread in native grasslands of the Medicine Lake sandplains than previously known, however we were unable to survey their habitats extensively at the right times to make such a determination.

One other species that had been recommended for addition to the state watch list has been dropped from consideration based on this study. We found rush aster (Aster junciformis) to be present in the various peatland habitats of the county, and common in many of them. It has a distribution in Montana much like Kalm's lobelia (Lobelia kalmii), in the northwest and northeast corners, but is known from more stations and a much wider range of habitats.

Two wetland plant species of special concern that were historically known from the Medicine Lake 
NWR were sought and not found. They are palespike lobelia (Lobelia spicata), which we did find on the Missouri Coteau, and slender bulrush (Scirpus heterochaetus). Neither did we relocate many-headed sedge (Carex sychnocephala) near Comertown, a wetland plant which had been collected little more than a decade ago, or have the correct location information to search for northern blue-eyed-grass (Sisyrinchium septentrionale), another wetland plant.

Though not in Sheridan County, American germander (Teucrium canadense) was collected at a Waterfowl Production Area nearby in Roosevelt County. This native species had previously been "reported" for Montana according to Dorn (1984), and we added it to the state watch list for further evaluation.

The fifteen significant plant species that we identified are profiled in Appendix G, presented in alphabetical order by scientific name. The information includes description of each species, its distribution in the state and the county, its habitat in the state and the county, and comments that note any unresolved status questions, potential threats, or management information.

We compiled and augmented information on the known flora of the county as well (Appendix J), underscoring county biological diversity. There are over 530 species of vascular plants collected or reported from the county to date, and over $10 \%$ of these are species not previously documented in northeastern Montana.

\section{Conclusions}

The goal of this project was to document the diversity of natural vegetation and plant species of special concern in Sheridan County, and to clarify their distribution and significance within the county, the state, and the Great Plains. In the process of this work, we also identified three landscape areas within the county that support the most intact areas of native vegetation, as well as concentrations of significance species and vegetation types. One of these areas centers on the Medicine Lake NWR, and another on the Missouri Coteau prairie pothole area that has been a focus area for the USFWS conservation easement program. This reflects the value of the USFWS Refuge and easement programs in helping to conserve the county's prairie and wetland resources.

Information that we gathered has provided the basis for updating and documenting status ranks and for expanding and refining the vegetation classification for Montana as a whole. This study also suggests many opportunities that may exist to help maintain the county's special and diverse biological resources in concert with efforts to protect rangeland values, promote watershed management, provide hunting and other recreational opportunities, and control noxious weeds.

While rangelands have become increasingly fragmented, and pressures on ranchers continue or increase, there is growing emphasis on maintaining and enhancing rangeland soil, water, and other natural resources. Sheridan County has many areas with potential for showcasing the successful habitat management that supports its diverse flora and fauna, through collaboration among landowner and conservation programs that support the private landowner. We have included a cross-reference between plant associations and the county's range sites (Appendix G) for use in range management coordination, planning, and education, especially in the rolling prairie pothole landscape, alkali lake systems, and sandhills.

Approximately half of the Sheridan County plant species of special concern and plant associations identified in this study are wetland features. Even though surface connectivity between wetlands and 
the Big Muddy drainage is limited, wetlands and their vegetation components are a key part of watershed planning.

At present, noxious weed infestations are restricted in the county. While eight of Montana's noxious weeds are present, they are not pervasive in native rangeland. The Invaders Database (2000) lists Hoary Cress (Cardaria draba), Spotted Knapweed (Centaurea maculosa), Russian Knapweed (Centaurea repens), Canada Thistle (Cirsium arvense), Field Bindweed (Convolvulus arvensis), Leafy Spurge (Euphorbia esula), Dalmatian Toadflax (Linaria dalmatica), and Common Tansy (Tanacetum vulgare) as present in Sheridan County.

One of the biggest threats to rangeland values, watershed protection, many plant species of special concern and native plant associations is the establishment and spread of Leafy Spurge (Euphorbia esula). It is highly competitive and resilient, and reported to survive underground over many years of cultivation. It is present in the Big Muddy drainage, including the valleybottom, some of the adjoining ravines, Medicine Lake, and the adjoining sandhills. It is dispersed by water and wildlife, and has the potential to expand throughout the county, threatening rangeland values and the health of native species and landscapes.

A number of areas and features within the county need additional survey to better document their distribution and status in the county. They are listed and described below:

- In the Missouri Coteau prairie potholes area Expand survey to re-locate many-headed sedge (Carex sychnocephala) and northern blue-eyed grass (Sisyrinchium

septentrionale)

Expand survey and documentation of alkali lake plant associations

- In the Medicine Lake area

Expand survey to re-locate slender bulrush (Scirpus heterochaetus) and pale-spike lobelia (Lobelia spicata)

Expand survey of plains phlox (Phlox andicola)
There is also a need for further surveys that include but may also extend beyond Sheridan County:

- Expand the wetland vegetation sampling in the Missouri Coteau, including the Sheridan County segment, to better document the diversity and nature of prairie pothole wetlands in Montana.

- Complete wetland and grassland vegetation descriptions and keys for the county and the northern glaciated plains as a region. Emphasis should be placed on further documenting all plant associations that are restricted to the northern Missouri Coteau region, that are known in Montana only from Sheridan County, or which may be uncommon rangewide.

- Conduct baseline inventories of small mammals, key invertebrate groups, and reptiles and amphibians, with emphasis on species of special concern.

Further inventory and assessment will be needed to determine the full extent of "northern plains" grasslands, alkali lake systems, sandhills, and wetland complexes in the state and region, and whether those in Sheridan County are indeed the most extensive or highest quality of their types.

In summary, Sheridan County harbors 12 plant species of special concern that are rare strictly from a state perspective, and outstanding examples of many of the 42 plant associations that are present in the county. Fourteen of the plant associations are newly-described or at least new to the state. Areas of the county are remarkably intact, and range management, noxious weed control, and related conservation measures are important in the longterm to these areas that support concentrations of Sheridan County's unique biological diversity. 


\section{Literature C ited}

Aasheim, M. 1970. Sheridan's Daybreak. Printed in Plentywood, MT.

Booth, W. E. and J. C. Wright. 1959. Flora of Montana, Part I. Monocotyledons. Department of Botany, Montana State University, Bozeman.

Booth, W. E. 1966 ed. Flora of Montana, Part II. Dicotyledons. Department of Botany, Montana State University, Bozeman. 305 pp.

Christianson, E. A. The Wisconsinan deglaciation of southern Saskatchewan and adjacent areas. Can. Earth Sciences v. 1: 913-938.

Chadde, S. W., J. S. Shelly, R. J. Bursik, R. K. Mosely, A. G. Evenden, M. Mantas, F. Rabe and B. Heidel. 1998. Peatlands on National Forests of the Northern Rocky Mountains: ecology and conservation. Gen. Tech. Rep. RMRS-GTR-11. Ogden, UT: USDA, Forest Service, Rocky Mountan Research Station. 75 pp.

Cholewa, A. F. and D. M. Henderson. 1984. Biosystematics of Sisyrinchium Section Bermudia (Iridiaceae) of the Rocky Mountains. Brittonia 36(4): 342-363.

Clayton, L., S. R. Moran, and J. P. Bluemle. 1980. Explanatory text to accompany the geologic map of North Dakota. Rep. No. 69. North Dakota Geol. Surv. 98 pp.

Cooper, S. V. and B. Heidel. 1998. Botanical and vegetation survey of Carter County, Montana. Unpublished report to Bureau of Land Management. Montana Natural Heritage Program, Helena. 116 pp. + app.

Cooper, S. V. and B. Heidel. 1999. Biodiversity and representativeness of research natural areas on national wildlife refuges in Montana. Unpublished report to U.S. Fish and Wildlife Service. Montana Natural Heritage Program, Helena. 63 pp. + app.

Coupland, R. T. 1950. Ecology of mixed prairie in Canada. Ecol. Monog. 20:271-315.
Coupland, R. T. 1960. A reconsideration of grassland classification in the northern Great Plains of North America. Ecology 49(1):135-167.

Cowardin, L., V. Carter, F. Golet and E. LaRoe. 1979. Classification of wetlands and deepwater habitats of the United States. U.S. Fish and Wildlife Service, OSB-79-31. Washington, D.C.

DeVelice, R., S. V. Cooper, J. T. McGarvey, J. Lichthardt, and P. Bourgeron. 1995. Plant communities of northeastern Montana: a first approximation. Unpublished report to Bureau of Land Management. Montana Natural Heritage Program, Helena. 116 pp. + app.

Dodd, J. and R. Coupland. 1966. Vegetation of saline areas in Saskatchewan. Ecology 47(6): 958-968.

Dorn, R. D. 1984. Vascular Plants of Montana. Mountain West Publishing, Cheyenne, WY. 276 pp.

Duxbury, A. Fens: our glacial relicts. North Dakota Outdoors. Vol. XLIX, No. 10:13-17.

Faber-Langendoen, D., ed. 1999. Terrestrial vegetation of the midwestern United States. Compiled community characterizations abstract in draft form. Midwest Office of The Nature Conservancy, Minneapolis, Minn.

Great Plains Flora Committee 1977. Atlas of the Flora of the Great Plains. Iowa University Press, Ames. 600 pp.

Great Plains Flora Committee 1986. Flora of the Great Plains. University Press of Kansas, Lawrence. 1392 pp.

Grossman, D. H., D. Faber-Langendoen, A. S. Weakley, M. Anderson, P. Bourgeron, R. Crawford, K. Goodin, S. Landaal, K. Metzler, K. Patterson, M. Pyne, M. Reid, and L. Sneddon. 1998. Terrestrial vegetation of the United States. Vol. 1. The national vegetation classification system: development, status, and applications. Nature Conservancy, Arlington, VA.126 pp. 
Hansen, P. L. and G. R. Hoffman. 1988. The vegetation of the Grand River/Cedar River, Siox and Ashland Districts of the Custer National Forest: a habitat type classification. Gen. Tech. Rep. RM-157. USDA Forest Service, Rocky Mountain Forest and Range Expt. Stn., Fort Collins, CO. 68 pp.

Hansen, P. L., R.D. Pfister, K. Boggs, . J. Cook, J. Joy, and D.K Hinckley. 1995. Classification and management of Montana's riparian sites. Montana Forest and Conservation Experiment Station, School of Forestry, Misc. Pub. No. 43. University of Montana, Missoula. 646 pp.

Hanson, H. C. and W. Whitman. 1938. Characteristics of major grassland types in western North Dakota. Ecological Monographs 8:57-114.

Heidel, B. 1992. Biodiversity inventory of the National Wildlife Refuge system in North Dakota. North Dakota Natural Heritage Inventory, Bismarck. 124 pp.

Heidel, B. 1996. Noteworthy collections: Montana. Madroño 43(3):436-440.

Heidel, B. and K. Dueholm. 1994. Sensitive plant species survey of the Sioux District, Custer National Forest, Montana and South Dakota. Unpublished report to the Custer National Forest. Montana Natural Heritage Program, Helena.

Heidel, B. and H. Marriott. 1996. Sensitive plant species survey of the Ashland District, Custer National Forest, Powder River and Rosebud counties. Unpublished report to the Custer National Forest. Montana Natural Heritage Program, Helena. 94 pp.

Invaders Database. 2000. Invaders Database System. Posted at http://invader.dbs.umt.edu/ Division of Biological Sciences, University of Montana, Missoula.

Kantrud, H. A., G. L. Krapu, and G. A. Swanson.1989. Prairie basin wetlands of the Dakotas: a plant association profile. U.S. Fish Wildl. Serv. Biol. Rep. 85(7.28). 116 pp.
Kartesz, J. T. 1994. Vol. 1. A synonymized checklist of the vascular flora of the United States, Canada, and Greenland. Timber Press, Portland, OR 622 pp.

Kindscher, K. 1992. Medicinal wild plants of the prairies. University Press of Kansas, Lawrence. $340 \mathrm{pp}$.

Lesica, P. 1987a. Medicine Lake Sandhills. Unpublished report to the Nature Conservancy. 11 pp + app.

Lesica, P. 1987b. Comertown Pothole Prairie. Unpublished report to the Nature Conservancy. $13 \mathrm{pp}+\mathrm{app}$.

Lesica, P. 1987c. The vegetation and conservation status of upland hardwood forests in eastern Montana. Unpublished report to the Nature Conservancy.

Lesica, P., P. Husby and S. V. Cooper. 1998. Noteworthy collections: Montana. Madroño 45:328-330.

Lesica, P. and S. V. Cooper. 1999. Succession and disturbance in sandhill vegetation: constructing models for managing biological diversity. Conservation Biology 13(2): 293-302.

Lichvar, R. W., E. I. Collins, and D. H. Knight. 1985. Checklist of vascular plants for the Bighorn Canyon National Recreation Area, Wyoming and Montana. Great Basin Naturalist 45(4): 734-746.

Looman, J. 1981. The vegetation of the Canadian prairie provinces. III. Aquatic and semi-aquatic vegetation. Phytocenologia 9:473-497.

Looman, J. 1982. Prairie grasses, identified and described by vegetative characters. Research Station, Swift Current, Saskatchewan. Agriculture Canada Pub. 1413. 244 pp.

Metcalf, F. P. 1931. Wild duck foods of North Dakota lakes. USDA Tech. Bull. 221. 72 pp.

Montana Native Plant Society. 1993. Guidelines for collecting plants. Kelseya 6(3):4. 
Mueggler, W. F. and W. L. Stewart. 1980. Grassland and shrubland habitat types of western Montana. General Technical Report INT-66. USDA Forest Service, Intermountain Forest and Range Experiment Station, Ogden, UT. 154 pp.

Redman, R. E. 1975. Production ecology of grassland plant communities in western North $\mathrm{Da}$ kota. Ecological Monographs 45:83-106.

Reid, M., K. Schulz, M. Schindel, P. Comer, G. Kittel, and others (compilers). 1999. International classification of ecological communities: Terrestrial vegetation of the Western United States. Report from Biological Conservation Datasystem and Working Draft of July 1999. Association for Biodiversity Information/The Nature Conservancy, Western Resource Office, Community Ecology Group, Boulder, Colorado.

Richardson, R. E. and L. T. Hanson. 1977. Soil survey of Sheridan County, Montana. USDA Soil Conservation Service (NRCS). Bozeman.

Riparian and Wetland Research Program. 2000. Draft classification and management of riparian and wetland sites of the Saskatchewan Prairie Ecozone and parts of adjacent subregions. University of Montana - School of Forestry. Missoula. Posted at: http://www.rwrp.umt.edu/

Ross, R. L., B. A. Andrews, and I. J. Witkind. 1955. Geologic map of Montana. U.S. Geological Survey, Washington, D.C.

Ross, R. L. and H. E. Hunter. 1976. Climax vegetation of Montana based on soils and climate. UDSA Soil Conservation Service, Bozeman, MT. 64 pp.

Scoggan, H. J. 1978. The Flora of Canada. Part 2 Pteridophyta, Gymnospermae, Monocotyledoneae. National Museum of Natural Sciences, Publications in Botany, No. 7(2). $545 \mathrm{pp}$.

Severson, K. E. and C. E. Boldt. 1977. Problems associated with management of native woody plants in the western Dakotas. Proceedings of the Sixth Wyoming Shrub Ecology Workshop, Buffalo, Wyoming.
Smith, R. 1976. Ecological and use information for plant species of the Aberdeen and Billings Areas of the Bureau of Indian Affairs. Report No. 241, Billings.

Stewart, R. E. and H. A. Kantrud. 1971. Classification of natural ponds and lakes in the glaciated prairie region. USDI Fish and Wildlife Service, Bureau of Sport Fisheries and Wildlife. Res. Pub. 92, Washington.

Stewart, R. E. and H. A. Kantrud. 1972. Vegetation of prairie potholes, North Dakota, in relation to quality of water and other environmental factors. U.S. Geological Survey Professional Paper 585D.

Thompson, W. 1994. Relationship of the national wetlands inventory to an ecological site classification of wetlands in northeast Montana. Masters Thesis. University of Montana, Missoula. 60 pp.

Umbanhowar Jr., C.E. 1996. Recent fire history of the northern Great Plains. Am. Midl. Nat. 135: 115-121.

Wallis, C. 1990. Reconnaissance survey of saline wetlands and springs in the Grassland-Parkland Region of eastern Alberta. Unpublished report to World Wildlife Fund Canada. Cottonwood Consultants Ltd. Calgary, AB.

Witkind, I. J. 1959. Quaternary geology of the Smoke Creek - Medicine Lake - Grenora Area, Montana and North Dakota. U.S. Geol. Surv. Bull. 1073, Washington, D. C. U.S. Govt. Printing Off. $80 \mathrm{pp}$.

USDI Fish and Wildlife Service. 1994. National list of plant species that occur in wetlands - Region 4, 5, and 8. Resource Management Group, Inc. Grand Haven, MI. 64 pp.

USDA Forest Service. 1992. Ecological sites and habitat types of the Little Missouri National Grassland and western North Dakota. USDA Forest Service, Custer National Forest, Billings, MT and Northern Region, Missoula, MT. 


\section{A ppendix A. Animal species of special concern in Sheridan County}

\begin{tabular}{|c|c|c|c|c|}
\hline Scientific name & Common name & $\begin{array}{l}\text { Global } \\
\text { Rank }\end{array}$ & $\begin{array}{l}\text { State } \\
\text { Rank }\end{array}$ & Status \\
\hline \multicolumn{5}{|l|}{ Reptiles and Amphibians } \\
\hline Rana pipiens & Northern Leopard Frog & G5 & S3S4 & Tracked \\
\hline Liochlorophis vernalis & Smooth Green Snake & G5 & S2S3 & Tracked \\
\hline Heterodon nasicus & Western Hognose Snake & G5 & S3 & Tracked \\
\hline \multicolumn{5}{|l|}{ Birds } \\
\hline Pelecanus erythrorhynchos & American White Pelican & G3 & S2B & Tracked \\
\hline Ardea herodias & Great Blue Heron & G5 & S4B & Watch \\
\hline Nycticorax nycticorax & Black-crowned Night-heron & G5 & S2S3B & Tracked \\
\hline Buteo regalis & Ferruginous Hawk & G4 & S3B & Tracked \\
\hline Coturnicops noveboracensis & Yellow Rail & G4 & S1B & Tracked \\
\hline Charadrius melodus & Piping Plover & G3 & S2B & Tracked \\
\hline Larus pipixcan & Franklin's Gull & G4G5 & S3B & Tracked \\
\hline Sterna caspia & Caspian Tern & G5 & S2B & Tracked \\
\hline Sterna forsteri & Forster's Tern & G5 & S2B & Tracked \\
\hline Chlidonias niger & Black Tern & G4 & S3B & Tracked \\
\hline Athene cunicularia & Burrowing Owl & G4 & S3B & Tracked \\
\hline Anthus spragueii & Sprague's Pipit & G4 & S4B & $\begin{array}{l}\text { Proposed } \\
\text { for Watch }\end{array}$ \\
\hline Lanius ludovicianus & Loggerhead Shrike & G5 & S4B & Watch \\
\hline Ammodramus bairdii & Baird's Sparrow & G4 & S3S4B & Tracked \\
\hline Ammodramus leconteii & LeConte's Sparrow & G4 & S1S2B & Tracked \\
\hline Ammodrammus nelsoni & Nelson's Sharp-tailed Sparrow & G5 & S1B & Tracked \\
\hline \multicolumn{5}{|l|}{ Insects } \\
\hline Hesperia ottoe & Ottoe Skipper & G3G4 & SU & Tracked \\
\hline Phycioides batesii & Tawny Crescent & G4 & S2S3 & Tracked \\
\hline
\end{tabular}


Appendix B. Equivalent scientific names for characteristic plants in Sheridan County.

\begin{tabular}{|c|c|c|c|}
\hline Common name & $\begin{array}{c}\text { In: Booth (1950), (1966) } \\
\text { or Great Plains Flora } \\
\text { Assoc. (1986) }\end{array}$ & In: Dorn (1984) & In: Kartesz (1994) \\
\hline $\begin{array}{l}\text { Thickspike } \\
\text { Wheatgrass }\end{array}$ & $\begin{array}{l}\text { Agropyron } \\
\text { dasystachyum }\end{array}$ & Elymus lanceolatus & Elymus lanceolatus \\
\hline $\begin{array}{l}\text { Western } \\
\text { Wheatgrass }\end{array}$ & Agropyron smithii & Elymus smithii & Pascopyrum smithii \\
\hline Little Bluestem & Andropogon scoparius & Schizachyrium scoparium & Schizachyrium scoparium \\
\hline $\begin{array}{l}\text { Bluebunch } \\
\text { Wheatgrass }\end{array}$ & Agropyron spicatum & Elymus spicatus & Pseudoroegneria spicata \\
\hline Sun Sedge & Carex heliophila & Carex pensylvanica & Carex inops \\
\hline Saltgrass & $\begin{array}{l}\text { Distichilis spicata var. } \\
\text { stricta }\end{array}$ & Distichilis stricta & Distichilis spicata \\
\hline $\begin{array}{l}\text { Few-headed } \\
\text { Spike-rush }\end{array}$ & Eleocharis pauciflora & Eleocharis pauciflora & Eleocharis quinueflora \\
\hline Common Milfoil & Myriophyllum spicatum & Myriophyllum spicatum & $\begin{array}{l}\text { Myriophyllum } \\
\text { excalbescens }\end{array}$ \\
\hline $\begin{array}{l}\text { Water } \\
\text { Smartweed }\end{array}$ & Polygonum coccineum & Polygonum amphibium & Polygonum amphibium \\
\hline $\begin{array}{l}\text { Shrubby } \\
\text { Cinquefoil }\end{array}$ & Potentilla fruticosa & Potentilla fruticosa & $\begin{array}{l}\text { Pentaphylloides } \\
\text { floribunda }\end{array}$ \\
\hline Lemon Scurfpea & Psolarea lanceolata & Psolarea lanceolata & Psoralidium lanceolatum \\
\hline $\begin{array}{l}\text { Northern } \\
\text { Porcupinegrass }\end{array}$ & Stipa curtiseta & $\begin{array}{l}\text { Stipa spartea } \\
\text { (implicit as } S . \text { s. var. } \\
\text { curtiseta) }\end{array}$ & Stipa curtiseta \\
\hline $\begin{array}{l}\text { Green } \\
\text { Needlegrass }\end{array}$ & Stipa viridula & Stipa viridula & Nasella viridula \\
\hline Sea Blite & Suaeda depressa & Suaeda calceoliformis & Suaeda calceoliformis \\
\hline
\end{tabular}




\section{Appendix C. Plant species inventory targets in Sheridan County}

\begin{tabular}{|c|c|c|c|}
\hline Scientific Name & Common Name & Global Rank & State Rank ${ }^{1}$ \\
\hline Agastache foeniculum & Lavender Hyssop & G4G5 & SU WATCH \\
\hline Agrimonia gryposepala & Common Agrimony & G5 & SU WATCH \\
\hline Ammania coccinea & Scarlet Ammannia & G5 & S1 \\
\hline Amorpha canescens & Leadplant & G5 & $\mathrm{SH}$ \\
\hline Anemone quinquefolia & Wood Anemone & & \\
\hline Asclepias ovalifolia & Ovalleaf Milkweed & & \\
\hline Aster frondosus & Alkali Aster & G4 & S1 \\
\hline Astragalus racemosus & Creamy Poison Vetch & G5T4 & S1 \\
\hline Bacopa rotundifolia & Waterhyssop & G5 & S1 \\
\hline Bidens frondosa & Leafy Beggarticks & G5 & SU WATCH \\
\hline Carex assiniboinensis & Assiniboin Sedge & & \\
\hline Carex bicknellii & Bicknell's Sedge & & \\
\hline Carex gravida & Pregnant Sedge & G5T5 & S1 \\
\hline Carex hallii & Hall's Sedge & & \\
\hline Carex meadii & Mead's Sedge & & \\
\hline Carex peckii & Peck Sedge & & \\
\hline Carex prairea & Brown-fruited Sedge & G5? & S1 \\
\hline Carex sychnocephala & Many-headed Sedge & G4 & S1 \\
\hline Carex tetanica & Tetan Sedge & & \\
\hline Celastrus scandens & American Bittersweet & G5 & S1 \\
\hline Centunculus minimus & Chaffweed & G5 & S2 \\
\hline Chenopodium subglabrum & Slimleaf Goosefoot & G3G4 & S1 \\
\hline Corylus americana & American Hazelnut & & \\
\hline Cryptantha fendleri & Fendler's Cryptantha & G4 & S1 \\
\hline Cyperus erythrorhizos & Redrooted Flatsedge & G5 & $\mathrm{SH}$ \\
\hline Cyperus odorata & Slender Flatsedge & & \\
\hline Cyperus rivularis & Shining Flatsedge & G5 & S1 \\
\hline Cyperus schweinitzii & Schweinitz's Flatsedge & G5 & S1 \\
\hline Dalea villosa var. villosa & Silky Prairie Clover & G5T? & S1 \\
\hline
\end{tabular}

\footnotetext{
${ }^{1}$ Species which have blanks in the rank columns are not known to occur in Montana, but they are reported in adjoining areas of North Dakota or Saskatchewan, and were considered in this study.
} 
Appendix C. (cont.)

\begin{tabular}{|c|c|c|c|}
\hline Scientific Name & Common Name & Global Rank & State Rank ${ }^{1}$ \\
\hline Desmodium canadense & Canada Tickclover & & \\
\hline Eleocharis acicularis & Needle Spikerush & & \\
\hline Eleocharis compressa & Flatstem Spikerush & & \\
\hline Eleocharis obtusata & Blunt Spikerush & & \\
\hline Elodea longivaginata & Longsheath Waterweed & G4G5 & S1 \\
\hline Eupatorium maculatum & Joepye Weed & G5 & S1 \\
\hline Gentianopsis macounii & Macoun's Gentian & G5 & S1 \\
\hline Gerardia aspera & Rough Gerardia & & \\
\hline Gerardia tenuiflora & Slenderleaf Gerardia & & \\
\hline Geum canadense & White Avens & G5 & SU WATCH \\
\hline Hutchinsia procumbens & Hutchinsia & G5 & S1 \\
\hline Hemicarpha drummondii & Drummond's Dwarf Bulrush & G4G5 & SU \\
\hline Hypoxis hirsuta & Yellow Stargrass & & \\
\hline Lathyrus palustris & Marsh Peavine & & \\
\hline Lobelia kalmii & Kalm's Lobelia & G5 & SU WATCH \\
\hline Lobelia spicata & Palespike Lobelia & G5 & S1 \\
\hline Mirabilis hirsuta & Hairy Four o'clock & G5 & S3 \\
\hline Penstemon angustifolius & Narrow Beardtongue & G5 & S2 \\
\hline Phacelia thermalis & Hot Spring Phacelia & G3G4 & S1 \\
\hline Phlox andicola & Moss Phlox & G4 & S1 \\
\hline Plagiobothrys leptocladus & Slenderbranched Popcornflower & G4 & S1 \\
\hline Polygala senega & Senica Snakeroot & & \\
\hline Polygonatum biflorum & Solomon's Seal & & \\
\hline Primula incana & Mealy Primrose & G5 & $\mathrm{S} 2$ \\
\hline Prunus pumila & Sand Cherry & G5 & S1 \\
\hline Psilocarphus brevissimus & Dwarf Woollyheads & G5 & $\mathrm{S} 1$ \\
\hline Ribes hirtellum & Hairy Gooseberry & & \\
\hline Rudbeckia hirta (R. serotina) & Blackeyed Susan & & \\
\hline Salix petiolaris & Meadow Willow & & \\
\hline
\end{tabular}

\footnotetext{
${ }^{1}$ Species which have blanks in the rank columns are not known to occur in Montana, but they are reported in adjoining areas of North Dakota or Saskatchewan, and were considered in this study.
} 
Appendix C. (cont.)

\begin{tabular}{|l|l|c|c|}
\hline \multicolumn{1}{|c|}{ Scientific Name } & \multicolumn{1}{|c|}{ Common Name } & Global Rank & State Rank \\
\hline Scirpus heterochaetus & Slender Bulrush & G5 & S1 \\
\hline Senecio congestus & Swamp Ragwort & G5 & S1 \\
\hline Sisyrinichium angustifolium & Northern Blue-eyed Grass & G3G & S1 \\
\hline Solidago sparsiflora & Threenerved Goldenrod & & \\
\hline Solidago speciosa & Showywand Goldenrod & & \\
\hline Sorghastrum nutans & Indian Grass & & \\
\hline Spiranthes spp. & Ladiestresses & G5 & S1 \\
\hline Spiranthes cernua & Ladiestresses & G5 & SU WATCH \\
\hline Stellaria crassifolia & Thickleaved Starwort & & \\
\hline Teucrium canadense & American Germander & G5 & S1 \\
\hline Vernonia fasciculata & Western Ironweed & & \\
\hline Viburnum lentago & Nannyberry & & \\
\hline
\end{tabular}

\footnotetext{
${ }^{1}$ Species which have blanks in the rank columns are not known to occur in Montana, but they are reported in adjoining areas of North Dakota or Saskatchewan, and were considered in this study.
} 


\section{Appendix D. Summary table of Sheridan County plant associations}

\begin{tabular}{|c|c|c|c|c|c|c|c|}
\hline Scientific and common names of plant associations & \begin{tabular}{|c|} 
Global \\
Rank \\
\end{tabular} & \begin{tabular}{|l} 
State \\
Rank \\
\end{tabular} & $\begin{array}{c}\text { \# of } \\
\text { Sample } \\
\text { Plots } \\
\end{array}$ & 总吾 & 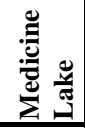 & 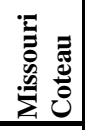 & ప⿱艹⿹勹巳 \\
\hline \multicolumn{8}{|l|}{ Woodland } \\
\hline Fraxinus pennsylvanica / Prunus virginiana Woodland & G3? & $\mathrm{S} 2 \mathrm{~S} 3$ & 2 & $\mathrm{X}$ & & & $\mathrm{X}$ \\
\hline \multicolumn{8}{|l|}{ Green Ash / Common Chokecherry Woodland } \\
\hline Populus tremuloides / Symphoricarpos albus Woodland & G3? & S3? & 1 & & & & $\mathrm{X}$ \\
\hline \multicolumn{8}{|l|}{ Quaking Aspen / Common Snowberry Woodland } \\
\hline \multicolumn{8}{|l|}{ Shrubland } \\
\hline Artemisia cana / Pascopyrum smithii Shrubland & G4 & S4 & 2 & $\mathrm{X}$ & & & $\mathrm{X}$ \\
\hline \multicolumn{8}{|l|}{ Silver sagebrush - Western Wheatgrass Shrubland } \\
\hline Elaeagnus commutata Shrubland & G2? & S2? & 1 & & $\mathrm{X}$ & & \\
\hline \multicolumn{8}{|l|}{ Silverberry Shrubland } \\
\hline Eriogonum pauciflorum - Gutierrezia sarothrae Badlands Sparse Vegetation & G? & S? & 0 & $\mathrm{X}$ & & & \\
\hline \multicolumn{8}{|l|}{ Few-flowered Wild Buckwheat - Broom Snakeweed Badlands Sparse Vegetation } \\
\hline Prunus virginiana Shrubland & G4Q & S4 & 0 & & $\mathrm{X}$ & & \\
\hline \multicolumn{8}{|l|}{ Common Chokecherry Shrubland } \\
\hline Sarcobatus vermiculatus / Pascopyrum smithii Shrub Herbaceous Vegetation & G4 & S4 & 1 & $\mathrm{X}$ & & & \\
\hline \multicolumn{8}{|l|}{ Black Greasewood / Western Wheatgrass Shrub Herbaceous Vegetation } \\
\hline Shepherdia argentea Shrubland & G3G4 & S3? & 0 & $\mathrm{X}$ & & & \\
\hline \multicolumn{8}{|l|}{ Buffaloberry Shrubland } \\
\hline Symphoricarpos occidentalis Shrubland & G4G5 & S4S5 & 0 & & $\mathrm{X}$ & $\mathrm{X}$ & \\
\hline \multicolumn{8}{|l|}{ Western Snowberry Shrubland } \\
\hline \multicolumn{8}{|l|}{ Herbaceous } \\
\hline Calamovilfa longifolia - Stipa comata Herbaceous Vegetation & G3 & $S ?$ & 1 & & $\mathrm{X}$ & & $\mathrm{X}$ \\
\hline \multicolumn{8}{|l|}{ Prairie Sandreed - Needle-and-thread Herbaceous Vegetation } \\
\hline Carex atherodes Herbaceous Vegetation & G3G5 & $S ?$ & 1 & & & $\mathrm{X}$ & \\
\hline \multicolumn{8}{|l|}{ Awned Sedge Herbaceous Vegetation } \\
\hline Carex lanuginosa Herbaceous Vegetation & G3? & S? & 0 & & $\mathrm{X}$ & $\mathrm{X}$ & $\mathrm{X}$ \\
\hline \multicolumn{8}{|l|}{ Woolly Sedge Herbaceous Vegetation } \\
\hline Carex praegracilis Herbaceous Vegetation & G3 & $\mathrm{S} ?$ & 6 & $\mathrm{X}$ & $\mathrm{X}$ & $\mathrm{X}$ & \\
\hline \multicolumn{8}{|l|}{ Clustered Field Sedge Herbaceous Vegetation } \\
\hline Distichlis spicata Herbaceous Vegetation & G5 & S4 & 0 & & & $\mathrm{X}$ & \\
\hline \multicolumn{8}{|l|}{ Saltgrass Herbaceous Vegetation } \\
\hline Eleocharis palustris Herbaceous Vegetation & G5 & S5 & 1 & & & $\mathrm{X}$ & \\
\hline \multicolumn{8}{|l|}{ Common Spike-rush Herbaceous Vegetation } \\
\hline Eleocharis quinqueflora Marl Fen Herbaceous Vegetation & G1? & S? & 4 & $\mathrm{X}$ & $\mathrm{X}$ & $\mathrm{X}$ & \\
\hline \multicolumn{8}{|l|}{ Few-flowered Spike-rush Marl Fen Herbaceous Vegetation } \\
\hline Elymus lanceolatus - (Koeleria macrantha) Herbaceous Vegetation & G? & S? & 11 & & & $\mathrm{X}$ & \\
\hline \multicolumn{8}{|l|}{ Thick-spike Wheatgrass - Prairie Junegrass Herbaceous Vegetation } \\
\hline Elymus lanceolatus - Stipa comata Northern Great Plains Herbaceous Vegetation & G? & S? & 9 & $\mathrm{X}$ & & $\mathrm{X}$ & $\mathrm{X}$ \\
\hline Thick-spike Wheat grass - Needle-and-thread Northern Great Plains Herbaceous & & & & & & & \\
\hline Hordeum jubatum Herbaceous Vegetation & G4 & S4 & 0 & & & $\mathrm{X}$ & \\
\hline Foxtail Barley Herbaceous Vegetation & & & & & & & \\
\hline Oryzopsis hymenoides - Psoralidium lanceolatum Herbaceous Vegetation & G3Q & $\mathrm{S} ?$ & 2 & & $\mathrm{X}$ & & \\
\hline Indian Ricegrass - Lemon Scurfpea Herbaceous Vegetation & & & & & & & \\
\hline Panicum virgatum - Muhlenbergia richardsonis - Schizachyrium scoparium & G? & S? & 2 & & $\mathrm{X}$ & $\mathrm{X}$ & $\mathrm{X}$ \\
\hline Switchgrass - Mat Muhly - Little Bluestem Herbaceous Vegetation & & & & & & & \\
\hline Pascopyrum smithii Herbaceous Vegetation & G3G5Q & S4 & 3 & $\mathrm{X}$ & $\mathrm{X}$ & & \\
\hline Western Wheatgrass Herbaceous Vegetation & & & & & & & \\
\hline Pascopyrum smithii - Distichilis spicata Herbaceous Vegetation & G4 & $S ?$ & 1 & $\mathrm{X}$ & & $\mathrm{X}$ & \\
\hline Western Wheatgrass - Saltgrass Herbaceous Vegetation & & & & & & & \\
\hline
\end{tabular}




\begin{tabular}{|c|c|c|c|c|c|c|c|}
\hline S cientific and common names of plant associations & \begin{tabular}{|c} 
Global \\
Rank
\end{tabular} & $\begin{array}{l}\text { State } \\
\text { Rank }\end{array}$ & $\begin{array}{c}\text { \# of } \\
\text { Sample } \\
\text { Plots }\end{array}$ & 疍 & 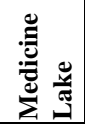 & 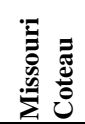 & ప্ّ \\
\hline Pascopyrum smithii - Stipa comata Northern Herbaceous Vegetation & G4 & S? & 4 & & $\mathrm{X}$ & & \\
\hline \multicolumn{8}{|l|}{ Western Wheatgrass - Needle-and-thread Northern Herbaceous Vegetation } \\
\hline Pascopyrum smithii - Stipa comata - Bouteloua gracilis Herbaceous Vegetation & G4 & S4 & 7 & $\mathrm{X}$ & $\mathrm{X}$ & & $\mathrm{X}$ \\
\hline \multicolumn{8}{|l|}{ Western Wheatgrass - Needle-and-thread - Blue Grama Herbaceous Vegetation } \\
\hline Pascopyrum smithii - Nassella viridula Herbaceous Vegetation & G4 & S4 & 1 & $\mathrm{X}$ & & & \\
\hline \multicolumn{8}{|l|}{ Western Wheatgrass - Green Needlegrass Herbaceous Vegetation } \\
\hline Polygonum amphibium Herbaceous Vegetation & G3? & S? & 0 & & & $\mathrm{X}$ & \\
\hline \multicolumn{8}{|l|}{ Water Smartweed Herbaceous Vegetation } \\
\hline Potamogeton pectinatus - Myriophyllum spicatum Herbaceous Vegetation & G3G4 & S1Q & 0 & $\mathrm{X}$ & $\mathrm{X}$ & $\mathrm{X}$ & $\mathrm{X}$ \\
\hline \multicolumn{8}{|l|}{ Sago Pondweed - Common Water-milfoil Herbaceous Vegetation } \\
\hline Puccinellia nuttalliana Herbaceous Vegetation & G3? & S? & 4 & & $\mathrm{X}$ & $\mathrm{X}$ & \\
\hline \multicolumn{8}{|l|}{ Nuttall's Alkaligrass Herbaceous Vegetation } \\
\hline Ruppia maritima Great Plains Herbaceous Vegetation & G? & S? & 1 & & $\mathrm{X}$ & $\mathrm{X}$ & \\
\hline \multicolumn{8}{|l|}{ Widgeon-grass Great Plains Herbaceous Vegetation } \\
\hline Salicornia rubra Herbaceous Vegetation & $\mathrm{G} 2$ & S2? & 1 & & & $\mathrm{X}$ & \\
\hline \multicolumn{8}{|l|}{ Red Glasswort Herbaceous Vegetation } \\
\hline Schizachyrium scoparium - Muhlenbergia cuspidata Herbaceous Vegetation & G3? & S2 & 2 & & & & $\mathrm{X}$ \\
\hline \multicolumn{8}{|l|}{ Little Bluestem - Plains Muhly Herbaceous Vegetation } \\
\hline Scirpus acutus Herbaceous Vegetation & G5 & S5 & 0 & & $\mathrm{X}$ & $\mathrm{X}$ & \\
\hline \multicolumn{8}{|l|}{ Hardstem Bulrush Herbaceous Vegetation } \\
\hline Scirpus maritimus Herbaceous Vegetation & G4 & S4 & 2 & & & $\mathrm{X}$ & \\
\hline \multicolumn{8}{|l|}{ Alkali Bulrush Herbaceous Vegetation } \\
\hline Scirpus pungens Herbaceous Vegetation & G3G4 & S3 & 1 & $\mathrm{X}$ & $\mathrm{X}$ & $\mathrm{X}$ & \\
\hline \multicolumn{8}{|l|}{ Common Threesquare Herbaceous Vegetation } \\
\hline Scolochloa festucacea Herbaceous Vegetation & G4G5 & S? & 1 & & & $\mathrm{X}$ & \\
\hline \multicolumn{8}{|l|}{ Sprangletop Herbaceous Vegetation } \\
\hline Spartina pectinata Herbaceous Vegetation & G3? & S? & 2 & & $\mathrm{X}$ & & $\mathrm{X}$ \\
\hline \multicolumn{8}{|l|}{ Prairie Cordgrass Herbaceous Vegetation } \\
\hline Stipa comata - Bouteloua gracilis Herbaceous Vegetation & G5 & S5 & 8 & $\mathrm{X}$ & $\mathrm{X}$ & & $\mathrm{X}$ \\
\hline \multicolumn{8}{|l|}{ Needle-and-thread - Blue Grama Herbaceous Vegetation } \\
\hline Stipa comata - Psoralidium lanceolatum Herbaceous Vegetation & G? & S? & 4 & & $\mathrm{X}$ & & \\
\hline \multicolumn{8}{|l|}{ Needle-and-thread - Lemon Scurfpea Herbaceous Vegetation } \\
\hline Stipa curtiseta Herbaceous Vegetation & G? & S? & 7 & & $\mathrm{X}$ & $\mathrm{X}$ & $\mathrm{X}$ \\
\hline \multicolumn{8}{|l|}{ Northern Porcupinegrass Herbaceous Vegetation } \\
\hline Triglochin maritimum Herbaceous Vegetation & $\mathrm{GU}$ & S? & 0 & & $\mathrm{X}$ & $\mathrm{X}$ & $\mathrm{X}$ \\
\hline \multicolumn{8}{|l|}{ Common Arrow-grass Herbaceous Vegetation } \\
\hline Typha latifolia Western Herbaceous Vegetation & G5 & S5 & 0 & & & $\mathrm{X}$ & \\
\hline \multicolumn{8}{|l|}{ Common Cattail Western Herbaceous Vegetation } \\
\hline \multicolumn{8}{|l|}{ Undescribed types } \\
\hline Calamagrostis stricta Herbaceous Vegetation & & & 0 & & & $\mathrm{X}$ & \\
\hline Narrow-spiked Reedgrass Herbaceous Vegetation & & & & & & & \\
\hline Carex aquatilis Great Plains Herbaceous Vegetation & & & 1 & & $\mathrm{X}$ & $\mathrm{X}$ & \\
\hline Water Sedge Herbaceous Vegetation & & & & & & & \\
\hline Crataegus chrysocarpa Woodland & & & 0 & & & & $\mathrm{X}$ \\
\hline Yellow-fruit Hawthorn Woodland & & & & & & & \\
\hline Pentaphylloides floribunda / Stipa curtiseta Shrubland & & & 1 & $\mathrm{X}$ & & & \\
\hline Shrubby Cinquefoil / Northern Porcupinegrass Shrubland & & & & & & & \\
\hline Elymus lanceolatus - Bouteloua gracilis Herbaceous Vegetation & & & 1 & & & $\mathrm{X}$ & \\
\hline Thick-spike Wheat grass - Blue Grama Herbaceous Vegetation & & & & & & & \\
\hline
\end{tabular}




\section{Appendix D. (cont.)}

\begin{tabular}{|c|c|c|c|c|c|c|c|}
\hline S cientific and common names of plant associations & $\begin{array}{c}\text { Global } \\
\text { Rank }\end{array}$ & $\begin{array}{l}\text { State } \\
\text { Rank }\end{array}$ & $\begin{array}{c}\text { \# of } \\
\text { Sample } \\
\text { Plots }\end{array}$ & 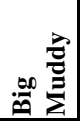 & 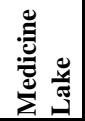 & 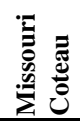 & ప̃ \\
\hline Elymus trachycaulus Herbaceous Vegetation & & & 1 & & & $\bar{X}$ & \\
\hline Bearded Wheat grass Herbaceous Vegetation & & & & & & & \\
\hline Elymus trachycaulus - Distichlis spicata Herbaceous Vegetation & & & 3 & & & $\mathrm{X}$ & \\
\hline Bearded Wheatgrass - Saltgrass Herbaceous Vegetation & & & & & & & \\
\hline Elymus trachycaulus - Spartina gracilis Herbaceous Vegetation & & & 1 & & & $\mathrm{X}$ & \\
\hline Bearded Wheatgrass - Alkali Cordgrass Herbaceous Vegetation & & & & & & & \\
\hline Juniperus horizontalis / Elymus lanceolatus Shrubland & & & 2 & $\bar{X}$ & & & \\
\hline Creeping Juniper / Thick-spike Wheatgrass Shrubland & & & & & & & \\
\hline Panicum virgatum - Spartina pectinata Herbaceous Vegetation & & & 1 & & & & $\mathrm{X}$ \\
\hline Switchgrass - Prairie Cordgrass Herbaceous Vegetation & & & & & & & \\
\hline Pascopyrum smithii - Bouteloua gracilis Herbaceous Vegetation & & & 5 & $\bar{X}$ & & & \\
\hline Western wheatgrass -Blue Grama Herbaceous Vegetation & & & & & & & \\
\hline Sarcobatus vermiculatus / Distichlis spicata - (Puccinellia nuttalliana) Saline Shrub & & & 1 & & & $\mathrm{X}$ & \\
\hline Black Greasewood / Saltgrass - (Nuttall's Alkaligrass) Saline Shrub Herbaceous & & & & & & & \\
\hline Scirpus nevadensis Herbaceous Vegetation & & & 2 & & $\bar{X}$ & $\bar{X}$ & \\
\hline Nevada Bulrush Herbaceous Vegetation & & & & & & & \\
\hline Spartina gracilis Herbaceous Vegetation & & & 1 & & & $\mathrm{X}$ & \\
\hline Alkali Cordgrass Herbaceous Vegetation & & & & & & & \\
\hline Stipa comata - Muhlenbergia cuspidata Herbaceous Vegetation & & & 1 & $\mathrm{X}$ & & & \\
\hline Needle-and-Thread - Plains Muhly Herbaceous Vegetation & & & & & & & \\
\hline
\end{tabular}


Appendix E.

Sheridan C ounty

Plant Associations 
$\mathrm{P}$ lant associations are presented in eight sets. The first four are herbaceous. They are listed alphabetically by dominant species within each set.

- Characteristic Grassland Types

- Minor and Under Review Grassland Types

- Wetland Types

- Wetland Types Under Review

- Shrubland Types

- Shrubland Types Under Review

- Woodland Types

- Woodland Types Under Review

Types that are newly-proposed with documentation are automatically assigned state and global ranks with a question mark, pending review inside and outside the state. Types that do not fit existing classifications and do not have adequate documentation in this study to propose them for addition have state and global ranks omitted.

\section{C haracteristic G rassland Types}

Grasslands are the most extensive natural vegetation in Sheridan County historically and at present. The following plant associations are common or well-developed types that are characteristic of the county. Grasslands are also referred to as prairie or steppe. A few grass-dominated plant associations are wetland types, treated separately.

Native grass dominants are commonly characterized by stature, growth form, and photosynthetic pathway. The following plant associations include many complementary "species-pairs" of contrasting characteristics. Midheight grasses and short grasses are often paired together, hence the characterization of the prevailing plains vegetation as "mixed grass prairie." Bunch-forming grasses and rhizomatous grasses are often present together, also contributing to the pattern of grassland cover and enhancing water adsorption abilities. Cool-season grasses increase with importance at these northern latitudes, but warm-season grasses like blue grama are often present in abundance with peak photosynthetic efficiency at warm temperatures.
The most widespread Sheridan County grassland plant associations correspond within the silty range site, comprised of midgrass species with or without a shortgrass component. This is also among the most arable range sites and the most productive rangeland, present in fragments throughout the county. Sand prairie plant associations are restricted mainly to the Medicine Lake sandplains. We could only begin to characterize grasslands of fine-textured valley soils because this habitat has been widely converted and altered, with exception of the more salt-affected and poorly-drained settings. We had a dual focus of documenting the outstanding examples of widespread, representative grassland types and the best remaining examples of other well-developed types that are more restricted by nature or by land-use history.

\section{Elymus lanceolatus - Sti pa comata N orthern G reat Plains H erbaceous Vegetation}

\section{Thick-spike Wheatgrass - N eedle-and-thread $\mathrm{H}$ erbaceous V egetation \\ Global rank: G? State rank: S?}

Summary: The thick-spike wheatgrass - needleand-thread herbaceous vegetation is a productive prairie plant association found in the northern Great Plains provinces and areas of adjoining states in glaciated landscapes on well-drained slopes with fine-textured soils, and on gently rolling uplands of silt or silt loam. It may be restricted to the northeasternmost corner of Montana and adjoining glacial drift plains and the collapsed moraine of Saskatchewan and North Dakota where the topography is gentle, and may have been a prevalent plant association. It is a priority for exchanging vegetation and range management information with ecologists from North Dakota and Saskatchewan. It is presented in this study as a "new" plant association, though it has been previously sampled and treated as part of other plant associations.

In Sheridan County, it is a major part of the prairie pothole landscape mosaic in the northeastern corner, also scattered in the drift plains to the south and west and in limited areas elsewhere. It may have been one of the most widespread plant association types in the county. Most large tracts of it have been plowed. 
Vegetation: This plant association has a graminoid cover of 90-100\%, and both Elymus lanceolatus and Stipa comata usually have at least $30 \%$ of total graminoid cover. It is a midgrass plant association that has a minor component of short graminoids, most often including sedges (Carex stenophylla, $C$. filifolia) and traces of blue grama (Bouteloua gracilis). Species diversity is moderate compared to other grassland associations, and there are no forb species reaching their highest cover values here with the possible exception of white milkwort (Polygala alba), reflecting the "melting pot" nature of this plant association type. Other common associated species include Hood's phlox (Phlox hoodii), yarrow (Achillea millefolium), Pasqueflower (Anemone patens) and American vetch (Vicia americana).

Environment: The typical setting for this plant association type is on dry, well-drained upland slopes on fine-textured soils. Some of the best examples in Sheridan County were found on the more gently-rolling of Missouri Coteau prairie pothole areas, all mapped as part of Zahill-Williams-Dimmick complex, and on glacial drift with Lambert silty clay loams in upland escarpment areas in the southwestern portion of the county. It is not only a prevalent upland type but an intermediate feature along slope and topographic position gradients; common on toeslopes and the welldrained areas of bottomland along the Big Muddy Creek valley where the Havrelon silt loam contains a distinctly sandy component or inclusion.

Comments: This cool-season, mid-grass plant association is distiniguished by thick-spike wheatgrass (Elymus lanceolatus) as co-dominant, replacing western wheatgrass (Pascopyrum smithii) as the most widespread wheatgrass in the northern Missouri Coteau of northwestern North Dakota and northeastern Montana (Heidel pers. obs.). This vegetation phenomenon has not been published in the technical literature to our knowledge.

\section{Elymuslanceolatus- (K oderia macrantha) $H$ erbaceous Vegetation \\ Thick-spike Wheatgrass - Prairie J unegrass $\mathrm{H}$ erbaceous $\mathrm{V}$ egetation \\ Global rank: G?; State rank: S?}

Summary: The thick-spike wheatgrass - prairie junegrass herbaceous vegetation is a productive prairie plant association found in the northern Great Plains provinces and northernmost areas of adjoining states in glaciated landscapes on fine textured soils. It is prevailing vegetation on clay deposits occupying the beds of former glacial lakes in the provinces, and otherwise a mosaic component on the finely-textured clay loam and silt loam soils on outwash channels and till deposits in both states and provinces. It is presented in this study as a "new" plant association, though it has been previously sampled and described in related Canadian vegetation classification systems.

In Sheridan County, it is an integral part of the prairie pothole landscape mosaic in the northeastern corner, and scattered widely across the rest of the county on top of gentle hills and ridges, as well as on benches and terraces of glacial meltwater valleys. It has been plowed as it occurred on level terrain elsewhere.

Vegetation: This plant association has a graminoid cover of 70-100\% with Elymus lanceolatus making up at least half of total cover and is sometimes the sole dominant. Koeleria macrantha is present and variously comprises less than $1 \%$ to almost half of total cover. It is a midgrass plant association that has a minor component of short graminoids, most often including sedges (Carex stenophylla, $C$. filifolia) and traces of blue grama (Bouteloua gracilis). It usually appears dense and homogeneous, occasionally interrupted by relatively sparse pockets of forbs in patches. The number of different forb species and their abundance tend to be very low. The most common and characteristic associated species include blue flax (Linum lewisii), spiny goldenweed (Machaeranthera pinnatifida), and red globe-mallow (Sphaeralcea coccinea). Total forb canopy cover is less than $5 \%$.

This is one of the more species-poor upland associations in the study area, as indicated by mean number of species per vegetation sampling plot, yet it is also one of the most productive, and lends itself to both landscape structural heterogeneity and 
mosaic richness. Intact prairie pothole tracts may represent some of the best-developed and most extensive examples in Montana.

Environment: The typical environment for this plant association type has been characterized as lacustrine clay soils that were deposited as glacial lake beds (Coupland 1950, 1960). It was prevalent in this habitat but the landscape has been extensively broken in the provinces, and is poorly represented further south in Montana, where at one representative glacial lakebed site it appears that western wheatgrass (Pascopyrum smithii) achieves dominance with green needlegrass (Nasella viridula; Cooper and Heidel 1999).

The typical Canadian environment where it was prevalent provides a context for understanding its environment at the southern limits of its range. In the Missouri Coteau of Sheridan County, is widespread in mosaic and large patch patterns on collapsed moraine, where it is consistently present at high points on the landscape. It encompasses the near-level upland segments of the silty and clayey range sites. It is consistently found on Zahill series soils derived from clay loam glacial till; often in mosaic patterns combined with the Williams series on steeply rolling collapsed moraine simply mapped as Zahill-Williams complex, hilly, 15-25\% slope (Richardson and Hanson 1977). Despite the rolling nature of the landscape, this plant association is restricted to nearly level (0-5\%) upland hilltop positions where its extent is determined in large part by the degree that the landscape includes level terrain.

Comments: The two major species of wheatgrasses have the highest productivity among dominant mixed grass prairie species (Coupland 1950). Thick-spike wheatgrass achieves much higher density and canopy cover than western wheatgrass (Heidel pers. obs). Clipping studies have documented that the "wheatgrass-junegrass faciation" has the highest forage yield (tons acre) in the mixed grass prairie landscape (Coupland 1950). Where Elymus lanceolatus - (Koeleria macrantha) Herbaceous Vegetation makes up more than a trace of primary range, the task of maintaining or restoring its condition is important in planning livestock use while maintaining cover, productivity, and landscape diversity. It is subject to heavy litter build-up and declines in forb numbers under idle conditions.

\section{Oryzops shymenoi des- Psorali dium lanceolatum $\mathrm{H}$ erbaceous Vegetation \\ Indian Ricegrass - Lemon Scurfpea $\mathrm{H}$ erbaceous $V$ egetation Global rank: G3Q ; State rank: S?}

Summary: The indian ricegrass - lemon scurfpea herbaceous vegetation is a sparse grassland restricted to slopes and crests of sand dunes recently disturbed by wind erosion (Lesica 1987). It is the driest and earliest successional stage of grassland types on sandplains. It may be distributed in adjoining states and provinces where dunes formed, e.g., in association with glacial lakes. Within Montana, the Medicine Lake sandhills are the largest area where it is known. Even though it covers a very small area in the county, it is considered a defining point of reference in sandhills succession.

Vegetation: The sparse graminoid cover is dominated by Oryzopsis hymenoides, usually comprising $20 \%$ or less canopy cover. Sand dropseed (Sporobolus crytandrus) is present, and more abundant in this association than elsewhere on the landscape, comprising up to $10 \%$ cover. Other grasses present include sand bluestem (Andropogon hallii) and needle-and-thread (Stipa comata). Grass cover is often approached or exceeded by the forb cover of Psoralidium lanceolatum, with values of $20 \%$ or greater. Wild begonia (Rumex venosus) is also characteristic of this setting, and two rare species are almost restricted to this habitat, including Schweinitz' flatsedge (Cyperus schweinitzii) and smooth goosefoot (Chenopodium subglabrum). The Fendler cat's-eye (Cryptantha fendleri) is another rare plant that is present here, but it is not restricted to this plant association.

Environment: This plant association is found in blow-outs, and is the driest and earliest stage of succession making up the sands range site. There is no soil development, and almost all of the groundcover is bare, unconsolidated sand. This habitat is maintained by the forces of wind.

Comments: This plant association is affected by efforts to heal dune blowouts and stabilize sandhills. Crested wheatgrass (Agropyron cristatum) has been seeded and widely-established in some pastures of the Medicine Lake sandhills. 
Even in this harshest of sandhills grassland types, leafy spurge (Euphorbia esula) has become established, signifying the potential of this noxious species to occupy the full range of sandhills habitats barring intervention.

\section{Pascopyrum smithii - Sti pa comata - Boutel oua gracilis H erbaceous Vegetation \\ Western Wheatgrass - N eedle-and-thread - Blue Grama H erbaceous V egetation Global rank: G4; State rank: S4}

Summary: The western wheatgrass - needle-andthread - blue grama herbaceous vegetation is a widespread Great Plains type centered in North and South Dakota, Montana and Wyoming, extending into adjoining states and provinces. It is prevalent in unglaciated plains within the "mixed grass prairie zone," widespread across the unglaciated "shortgrass prairie zone," and common to prevalent in the glaciated Great Plains. It is codominated by Pascopyrum smithii, Stipa comata, and Bouteloua gracilis.

In Sheridan County, it is found on well-drained benches and terraces along Big Muddy and Wolf Creeks, and to some extent on the Flaxville Gravel uplands to the west. The uplands around nearby Homestead Lake NWR appear to have this association, though perhaps with more Koeleria macrantha than Pascopyrum smithii, and abundance of Artemisia biennis that may indicate a history of heavy grazing.

Vegetation: This plant association is co-dominated by mid- and short-height grasses, with generally high vegetation cover of $60-100 \%$, and intermediate productivity. Pascopyrum smithii and Stipa comata are mid-height grasses usually totaling over half of net canopy cover, and prairie junegrass (Koeleria macrantha) is also present in most stands, but usually contributing $10 \%$ or less cover. The cover of Bouteloua gracilis, a shortgrass species, is usually comparable or exceeding that of the midgrass species, sometimes replaced by thread-leaved sedge (Carex filifolia) or sun sedge (C. heliophila). Typical forbs include Hood's phlox (Phlox hoodii), red globe-mallow (Sphaeralcea coccinea), scarlet gaura (Gaura coccinea), white penstemon (Penstemon albidus), rush-like skeletonweed (Lygodesmia juncea), fringed sage
(Artemisia frigida), Nuttall's pussy-toes

(Antennaria microphylla), purple prairie clover (Dalea purpurea) and rough pennyroyal (Hedeoma hispidum). In southeastern Montana and northeastern Wyoming, stands of this association often contain big sagebrush (Artemisia tridentata ssp. wyomingensis) though at cover values too low for this association to be considered shrubland or shrub herbaceous vegetation.

Environment: This plant association is found on flat or gently sloping terrain ranging from valley toeslopes to uplands. Surface layers of soils are usually clay loams, although stands of this type may also be found on loams, silt loams, silty clays and clays (Hanson and Whitman 1938, Hansen and Hoffman 1988). In Alberta and Saskatchewan this association grows on loamy sands to sandy loams in the center of the plains (Coupland 1960).

Comments: The cover contribution of Pascopyrum smithii and Bouteloua gracilis have been found to vary inversely in periods of drought or high rainfall, so that plots originally read in drought years dominated by Stipa comata - Bouteloua gracilis were re-read over a decade later and determined to include western wheatgrass co-dominance (Coupland 1960). The midgrass component also declines under heavy grazing.

Exotic brome grasses, especially hairy brome (Bromus commutatus) and cheatgrass (B. tectorum), are present in many stands of this association and they commonly contribute substantial cover (Hanson and Dahl 1956, Hansen et al. 1984, Hansen and Hoffman 1988).

\section{Sti pa comata - Psoralidi um lanceolatum H erbaceous Vegetation \\ $\mathrm{N}$ eedle-and-thread - Lemon Scurfpea $\mathrm{H}$ erbaceous Vegetation Global rank: G?; State rank: S?}

Summary: The needle-and-thread - lemon scurfpea herbaceous vegetation is restricted to wind-blown sand deposits with undeveloped soils, spanning almost all topographic positions except swales. It is found on both choppy dunes and rolling plains. It is thought to represent a seral stage between the barely-stabilized open dune vegetation with indian ricegrass - lemon scurfpea plant association (Oryzopsis hymenoides - Psoralidium 
lanceolatum) and the western wheatgrass - needleand-thread plant association (Pascopyrum smithii Stipa comata; Lesica 1987a). It is presented in this study as a "new" type, though it has been previously reported in this earlier work, but not described or incorporated in the state classification to date.

The distribution may include dunes formed in association with glacial lakes in adjoining states and provinces. The Medicine Lake Sandhills are the largest setting where it is known from in Montana.

Vegetation: Grass cover, almost all of which is Stipa comata, is generally $20-40 \%$, and forb cover represented primarily by Psoralidium lanceolatum approaches or exceeds it. Despite comparatively high cover values, litter accumulation is low and bare soil is exposed at the surface (20-60\%). Characteristic species include Fendler cat's-eye (Cryptantha fendleri), purple prairie clover (Dalea purpurea), and western spiderwort (Tradescantia occidentalis). Grazing increasers are consistently present, but take on high cover values where grazing has induced shift in dominance. The increasers include biennial wormwood (Artemesia biennis), brittle pricklypear (Opuntia fragilis), and hoary aster (Machaeranthera canescens).

Environment: This plant association occurs on stabilized and semi-stabilized sandhills. The topography of the reworked sand is irregularly rolling to choppy, forming very many dips and rises. The Medicine Lake sandhills and fringes are mapped as Blanchard fine sands, 4-20 degrees slope, and the plant association represents the prevalent form of "sands range site" in the county. Soil profile development is limited and litter accumulation is low so that bare sand is visible at the surface. The affects of wind erosion are limited, baring concentrated use as around windmills, or major reductions in standing cover.

Comments: Medicine Lake sandhills infestations of leafy spurge (Euphorbia esula) occur within this type, at early stages of invasion.

\section{Sti pa curti seta H erbaceous Vegetation \\ N orthern Porcupinegrass $\mathrm{H}$ erbaceous Vegetation \\ Global rank: G?; State rank: S?}

Summary: The northern porcupinegrass herbaceous vegetation is an extremely productive prairie type of sheltered hillslopes in prairie pothole and prairie parkland regions of the provinces and adjoining northern Great Plains states. It is rich in species numbers, particularly forbs, and includes grasses and forbs that are restricted to or reaching their peak frequency in this plant association compared with surroundings. It corresponds with the Stipa spartea var. curtiseta - forb community described by Redmann (1975) from one plot on a north-facing slope, north of the Missouri River in western North Dakota.

In Sheridan County, it had been previously reported from Medicine Lake NWR (Cooper and Heidel 1999) under the plant association name for it from the Canadian Prairie Parklands, Stipa curtiseta Elymus lanceolatus Herbaceous Vegetation. It is presented in this study as a "new" plant association of the northern plains, building upon the earlier one-plot studies, and distinct at some level from the Stipa curtiseta - Elymus lanceolatus Herbaceous Vegetation of the Canadian Parklands.

Vegetation: The northern porcupinegrass herbaceous vegetation has over $70 \%$ cover of northern porcupinegrass (Stipa curtiseta), and total canopy cover approaching or exceeding $100 \%$ with the bunchgrass life-form giving it a densely-tufted appearance. Shrub cover ranges from 1-10\% and variably includes prairie rose (Rosa arkansana), western snowberry (Symphoricarpos occidentalis), and sometimes silverberry (Elaeagnus commutata). Small-winged sedge (Carex stenophylla) is usually present and spike-oat (Helictotrichon hookeri) is restricted to this plant association in the study area. Among the diverse forbs, pasqueflower (Anemone patens), old man's whiskers (Geum triflorum) and northern hedysarum (Hedysarum boreale) are particularly abundant. Remann (1975) describes a unique feature of this plant association as the abundance of moss understory in places, a phenomenon that was noted in this study as well. The bryophyte flora and role may be important to exam further. 
Environment: The typical setting for this plant association is on glacial till, usually restricted to cool, moist north-facing slopes with loamy soils. The most examples were found in the Missouri Coteau prairie pothole areas around Comertown, but it was also noted where glacial meltwater cut through till or outwash. Soils in the former are mapped as Zahill-Williams complex, hilly. The soils are well-drained, and mantled by a deep layer of litter exposing little or no bare soils. Some sites seemed to have such dense root networks as to form turf. The association is limited by both slope and aspect to relatively steep, mainly north-facing slopes of $10 \%$ or greater.

Comments: This plant association type may be the most productive of all upland grassland types in the study area. Northern Porcupine grass had the highest yield per basal area percentage compared to all other prairie grasses in the Canadian northern Great Plains except for rough fescue (Festuca scabrella; Coupland 1960), which was not found in the county. However, the awns of northern porcupine grass deter grazing during the early summer period of peak growth and yield according to ranchers.

Response to fire has been tested on Medicine Lake National Wildlife Refuge, warranting close investigation and documentation. Despite the consistent presence of woody vegetation, there were no sites observed where shrubs appeared to be encroaching and assuming dominance.

It was characterized as the "Stipa curtiseta Elymus lanceolatus Herbaceous Vegetation" in Cooper and Heidel (1999) from one station in Medicine Lake National Wildlife Refuge, following the convention of Canadian literature as a Parkland plant association in which wheatgrass cover approaches or exceeds needlegrass cover. We provisionally present it here as a separate plant association in considering the paucity of thickspike wheatgrass in all sample plots. The separate association of northern porcupinegrass - thick-spike wheatgrass is under research in Valley County (Cooper in prog.).

Classification Comments: Stipa curtiseta forms monodominant stands in the Missouri Coteau, whereas it is closely associated with Elymus lanceolatus on drift plains and escarpments. The Stipa curtiseta type is presented separately from the Elymus lanceolatus - Stipa curtiseta type because of its distinct species richness and composition, as well as distribution. The latter type is being documented in Valley County, after which further comparison and analysis is warranted. The only settings where Stipa curtiseta is reported as singlespecies dominant in Canada are on steep, dry slopes of the parkland zone.

\section{Minor and U nder R eview $G$ rassland Types}

The following types are characterized as "minor" in the parts of the county where study was focused, generally occupying small areas or with limited development. There are also types that are presented as under review which do not fit existing or proposed classifications.

\section{C alamovilfa longi folia - Stipa comata $H$ erbaceous Vegetation \\ Prairie Sandreed - N eedle-and-thread $\mathrm{H}$ erbaceous $V$ egetation Global rank: G3; State rank: S?}

Summary: The prairie sandreed - needle-andthread herbaceous vegetation occurs in the central Great Plains region, e.g., the Nebraska Sandhills, and less extensive to the north. Stands occur on stabilized sand dunes, as well as in interdunal valleys, and colluvial sands. Soils are medium to fine sands formed either from eolian or colluvial processes. The vegetation has an open canopy, dominated by mid- to tall grasses. Calamovilfa longifolia and Stipa comata are the most conspicuous and dominant grasses. Other common grasses include blue grama (Bouteloua gracilis), prairie junegrass (Koeleria macrantha), indian ricegrass (Oryzopsis hymenoides), and sand dropseed (Sporobolus cryptandrus).

It is included among the suite of plant associations making up the Medicine Lake sandhills, all mapped as Blanchard fine sands, 4-20 degree slopes; corresponding with sands range sites. It does not appear to be a widespread plant association in the Medicine Lake sandhills as originally postulated (Cooper and Heidel 1999), but occurs in small patches on low ridges and in poorly-sorted mosaic 
patterns on gentle plains of Medicine Lake surrounding sandplains under certain grazing regimes. One of the more extensive areas of it along Sand Creek (plot 007) was used for earlyseason grazing as a calving pasture, and had high cover of blue grama (Bouteloua gracilis.) With more soil development on sandy loams, it appears that Pascopyrum smithii - Stipa comata Herbaceous Vegetation prevails. It might otherwise be present around sandstone escarpments in the state, and Calamovilfa longifolia was noted in local abundance on sandstone west of Antelope in association with thread-leaved sedge (Carex filifolia). In any case, plant associations with prairie sandreed are not widespread in Sheridan County, and they warrant further evaluation in the state.

\section{Elymuslanceolatus - Bouteloua gradilis $H$ erbaceous Vegetation \\ Thick-spike Wheatgrass - Blue Grama $\mathrm{H}$ erbaceous $V$ egetation}

Summary: An upland area co-dominated by thickspike wheatgrass and blue grama occurred in silt loam uplands strewn with rock, above an alkaline lake. This unusual combination does not have a corresponding vegetation description, so it is noted here as an undescribed, provisional type under review. Some of the more stress-tolerant species present include Atriplex gardneri and Astragalus pectinatus.

\section{Pascopyrum smithi $i$ - Bouteloua gracilis $\mathbf{N}$ orthern Plains $\mathbf{H}$ erbaceous Vegetation Western Wheatgrass - Blue Grama $\mathrm{H}$ erbaceous Vegetation}

Summary: The western wheatgrass - blue grama herbaceous vegetation is provisionally identified as a northern Great Plains plant association of hot alluvial settings and thinsoil settings overlying shale that are saturated in spring but dry for most of the growing season. It corresponds with the Bouteloua-Agropyron Faciation of Coupland (1960). Western wheatgrass comprises at least $20 \%$ cover and blue grama cover is about twice as much as western wheatgrass cover. Species diversity is low, and the characteristic forbs include plains pricklypear (Opuntia polyacantha), yellow flax (Linum rigidum), and rough pennyroyal (Hedeoma hispida).

Sheridan County examples were documented in valleybottom settings along the Big Muddy Creek and in small areas of Sand Creek. It was also found to be locally common on the rolling uplands above alkali lakes. Though the latter is an upland setting, the soils are ustifluvents.

Classification comments: There is also a Pascopyrum smithii - Bouteloua gracilis plant association recognized from foothill and lowermontane valleys of southwestern states. The northern Great Plains examples are treated separately because of non-overlapping climate and setting. However, intervening examples and additional vegetation comparison may link these plant associations that are provisionally treated as distinct.

The Pascopyrum smithii - Bouteloua gracilis plant association grades into the Pascopyrum smithii Distichilis spicata plant association with increase in salinity. It grades into the Pascopyrum smithii plant association with intermittent flooding.

This plant association is typical of the clayey range site. Additional vegetation sampling is needed to document and describe it.

\section{Pascopyrum smithii - D istichlisspicata $H$ erbaceous Vegetation \\ Western Wheatgrass - Saltgrass H erbaceous $\checkmark$ egetation \\ Global rank: G 4; State rank: S?}

Summary: The western wheatgrass - saltgrass herbaceous vegetation has been described for mainly unglaciated landscapes of Wyoming, Nebraska, and North Dakota in depressions and on stream terraces on deep, moderately saline soils, sometimes with a clay subsoil and is also present in alkali lake systems of the prairie pothole region. It is treated as a wetland type, on soils that are wet for part of the year and may flood periodically. This plant association is dominated by graminoids, and 
most of the vegetation is $0.6 \mathrm{~m}$ or less. The dominants are Pascopyrum smithii and Distichlis spicata. Woody plants are minor species, but big sagebrush (Artemisia tridentata subsp.

Wyomingensis), silver sagebrush (Artemisia cana subsp. cana), sandbar willow (Salix exigua), and plains cottonwood (Populus deltoides) are present in some stands. Forbs that may be present are marsh elder (Iva annua), prairie sunflower (Helianthus petiolaris), indian-wheat (Plantago patagonica), broom snakeweed (Gutierrezia sarothrae), and white-prairie aster (Aster falcatus).

In Sheridan County, the best-developed examples were found at alkali lakes of the Missouri Coteau on rolling, salt-affected glacial outwash uplands where salts rise to the surface. Soils are silty clay ustifluvents or Zahill clay loam. This plant association is also present in small areas of the Big Muddy and Wolf Creek valleys where it is temporarily inundated in spring or after heavy rains. Some sites are clearly in upland settings, but more information is needed on soils and hydrology to determine whether they are wetland or terrestrial. Terrestrial forms may be more closely allied with the upland Distichilis spicata plant association. This plant association has low species diversity, and exotic species including yellow sweetclover (Melilotus officinalis), are present though only in trace amounts.

\section{Pascopyrum smithi $\mathrm{i}$ - $\mathrm{N}$ assella vi ridula $H$ erbaceous Vegetation \\ Western Wheatgrass - G reen N eedlegrass $\mathrm{H}$ erbaceous Vegetation \\ Global rank: G4; State rank: S4}

Summary: The western wheatgrass - green needlegrass plant association type is widespread across much of the northern Great Plains. Stands occur in narrow valleys, on stream terraces, and on some uplands (Jones 1992, USFS 1992). Soils are fine-textured, including clays, silty clays, clay loams, or rarely loams, and moderately-drained. The soil profile is typically well developed. The parent material is siltsone and mixed sedimentary rock (USFS 1992). This plant association usually occurs on level or nearly level ground but sometimes may be on moderate slopes of any aspect. Most of the documentation for this type comes from unglaciated landscapes, and its distribution and environmental attributes in glaciated settings need more work.

In Sheridan County, this is a minor type and was noted in only one valleybottom setting along Eagle Creek in the Big Muddy headwaters area. Most valleybottom settings for in the western part of the county are too dry or salt-affected for it. It represents the highly productive "overflow" range site.

\section{Pascopyrum smithi i - Sti pa comata N orthern $\mathbf{H}$ erbaceous Vegetation}

Western Wheatgrass - N eedle-and-thread $\mathrm{N}$ orthern $\mathrm{H}$ erbaceous V egetation

Summary: The western wheatgrass - needle-andthread northern herbaceous vegetation as expressed in Sheridan County occupies stable northern sandplains and medium-textured uplands on gentle and nearly-level slopes. It is a highly-productive vegetation and may have been a prevailing type on the drift plains. The poor competitive ability of blue grama (Bouteloua gracilis) in this northern setting is reflected in the great reduction in shortgrass components except in drought episodes (Coupland 1960).

In the study area, it is found at opposite corners of the county. It spans the gentle sandplain skirting the Medicine Lake sandhills, on Lihen loamy fine sand, 0-6 \% slope; and locally repeats in swales and flats within the sandhills. It is also found on Williams loam across the tablelands adjoining the Big Muddy headwaters. It may be an prevailing type, but surveys were incomplete for making this characterization and much of the habitat has been converted to cropland. 


\section{Schi zachyri um scopari um - (Muhlen bergia cuspi data) H erbaceous Vegetation}

\author{
Little Bluestem - Plains M uhly H erbaceous \\ Vegetation \\ Global rank: G3?; State rank: S2
}

terrain, at Medicine Lake on sandy loam at the highest point of Big Island, and along south-facing ravine slopes south of the Lake. Soils range from well-drained clay loams to sandy loams and "shallow to gravel range sites", and it is most commonly characterized as a "sandy range site".

\section{Sti pa comata - Muhlenbergi a auspi data $H$ erbaceous Vegetation N eedle-and-thread - Plains M uhly $\mathrm{H}$ erbaceous Vegetation}

Summary: Plant associations dominated by needle-and-thread and plains muhly occur on sparse, early-succession grassland on steep, exposed, fine-textured slopes. Total vegetation cover is usually less than $40 \%$. The cover of plains muhly is $10 \%$ or greater, sometimes comprising over half of total graminoid cover. Early succession shrubs are often present in trace amounts including skunkbush sumac (Rhus trilobata) and silver sagebrush (Artemisia cana). Characteristic forbs include shining penstemon (Penstemon nitidus), bastard toadflax (Commandra umbellata), dotted blazing-star (Liatris punctata), and round-leaved thermopsis (Thermopsis rhombifolia).

In Sheridan County, it was observed on the most exposed of ravine slopes along the upper Big Muddy valley, on silty-clay of the Lambert-Zahill complex, 20-50\%.

\section{Wetland Types}

Wetlands are common in parts of Sheridan County, and cover approximately $4 \%$ of the total county surface area (40,868 acres; USDI National Wetlands Inventory Statistics). The most extensive and certainly the most numerous of wetlands are palustrine types (Cowardin et al. 1979), i.e., shallow wetlands more commonly referred to as marshes, sloughs, or swales. This study focused on palustrine vegetation, and more particularly the emergent forms to a depth of $1 \mathrm{~m}$. Palustrine wetlands comprise $53 \%$ of the wetland area in the county (Figure F-1). Lacustrine wetlands include the largest lake in the county, Medicine Lake, and 


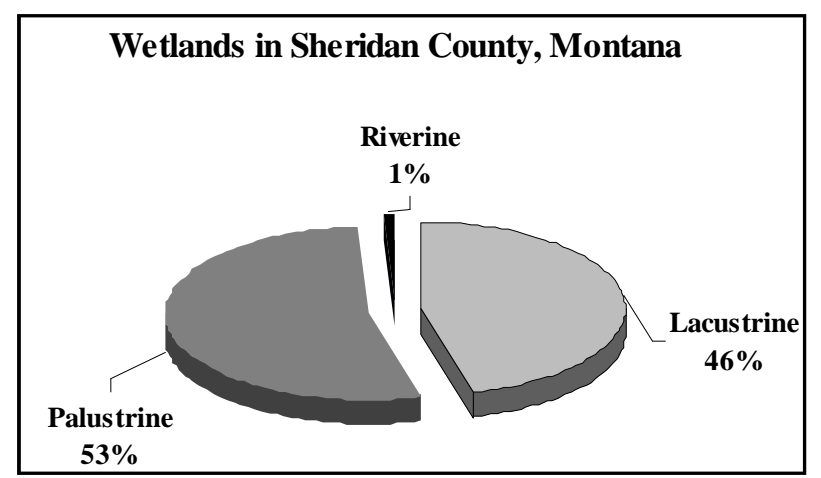

Figure F-1

the deepest lake in the county, Brush Lake. They represent openwater systems usually greater than 20 acres, and with water depth greater than $2 \mathrm{~m}$ or else having characteristic features like a waveformed shoreline (Cowardin et al. 1979). By this latter criteria, they also includes the most alkaline water bodies in the county. Lacustrine wetlands in the county total 18,776 acres. Riverine wetlands are restricted mainly to the Big Muddy Creek and total only 317 acres.

The plant associations are cross-referenced to the wetland types and vegetation types of Cowardin et al. (1979), Hansen et al. (1995), Stewart and Kantrud (1972), and Kantrud et al. (1989). Sample size is low for most wetland plant associations, but a provisional description is presented based on the literature. The reader is referred to schematic diagrams in the latter two references for visualizing the patterns of plant association distribution within and between wetlands, and the natural dynamics over time. Undescribed wetland types are presented separately at the end.

\section{C arex atherodes $\mathrm{H}$ erbaceous Vegetation}

\section{Awned Sedge $H$ erbaceous V egetation} Global rank: G 3G 5; State rank: S?

Summary: The awned sedge wet meadow occurs in the northern tallgrass and mixed grass prairie regions of the United States and Canada. Stands occur on lowland sites that are seasonally innundated for several weeks each year. These sites are typically in depressions or basins but can be along streams and rivers. The water may be fresh or moderately saline. Soils can be mineral but mucks often form through the buildup of organic material. Vegetation cover is usually high but can vary between wet or dry years. Dominant species are herbaceous and typically between 0.5 and $1 \mathrm{~m}$ tall. Forb diversity is moderate to high. Carex atherodes may form essentially monotypic stands or just be the dominant species. Commonly associated species include Americn waterplaintain (Alisma triviale), panicled aster (Aster lanceolatus),common spike-rush (Eleocharis palustris), American mannagrass (Glyceria grandis), wild mint (Mentha arvensis), reed canarygrass (Phalaris arundinacea), water smartweed (Polygonum amphibium), sprangletop (Scolochloa festucacea), hemlock water-parsley (Sium suave), and small bur-reed (Sparganium eurycarpum).

The awned sedge plant association is a common wetland plant association in the prairie provinces (Looman 1982, Riparian and Wetland Research Program 2000 ) as well as in the Missouri Coteau prairie potholes of Sheridan County, generally found in wetlands of less than 1-2 acres (Lesica 1987b.)

Comments: It was once a common source of "slough hay" (Looman 1982).

\section{Carex lanuginosa - C alamagrosti stricta $H$ erbaceous Vegetation \\ Woolly Sedge - N arrow-spiked Reedgrass $\mathrm{H}$ erbaceous $V$ egetation Global rank: G3?; State rank: S?}

Summary: The wooly sedge - narrow-spiked reedgrass herbaceous vegetation is found in the northern Great Plains. Stands occur on level ground in shallow depressions and other lowlands on poorly drained sandy, loamy, or silty clay soils. Standing water may be present for a few to several weeks during most years. Soil $\mathrm{pH}$ is circumneutral to somewhat alkaline, and organic content can be moderately high. The vegetation of this community provides approximately 100 percent graminoid cover, typically $0.3-0.6 \mathrm{~m}$ tall. Forb cover and diversity is low.

It is occasional in Missouri Coteau prairie potholes of Sheridan County. It appears to be situated in flow-through hydrological positions, with other wetlands situated both higher and lower in the watershed. 


\section{C arex praegracilis H erbaceous Vegetation \\ Clustered Field Sedge H erbaceous Vegetation \\ Global rank: G3; State rank: S?}

Summary: The clustered field sedge herbaceous vegetation is a wet meadow plant association of the Great Plains. The species is characterized as the only abundant native graminoid of "fresh" water conditions in the wet meadow zone of prairie pothole region (Stewart and Kantrud 1972), diminishing with increasing alkalinity. Using this information as inference, it is to be expected as the least-alkaline wet meadow plant association of shallow wetlands and the outer zones around deeper wetlands; sometimes associated with springs and seeps.

Vegetation: This plant association has $50-90 \%$ cover of Carex praegracilis, and typically occurs over limited area corresponding with small wetlands or narrow wetland zones. Other graminoid species sometimes present include tufted hairgrass (Deschampsia cespitosa), bearded wheatgrass (Elymus trachycaulus), meadow barley (Hordeum brachyantherum), and common arrowgrass (Triglochin maritimum). Commonly associated forbs include white-prairie aster (Aster falcatus) and lance-leaved goldenweed (Haplopappus lanceolatus).

Environment: The setting is temporarily flooded early in the growing season. More soils information is needed. Though most settings are mapped as part of fine-textured loams, the places where this occurs on the landscape appear to have porous, welldrained soils. The soils appeared to accumulate high organic content in some of the settings. The presence of halophytes like Triglochin maritimum and sea-milkwort (Glaux maritima) would seem to suggest that it is not necessarily restricted to places with fresh water conditions.

The Medicine Lake area has examples of this plant association along the Lake Creek valley, where in one place it was found to cover one side of the valleybottom. The Missouri Coteau prairie potholes had few small swales with this type. One of the more unusual examples was in the Big Muddy headwaters associated with seeps in the midst of a wooded slope; noted elsewhere in the Big Muddy valley margin and coulee bottoms.
Comments: This plant association occupies a zone that is readily invaded by exotics, and where livestock use is concentrated. Species that increase under disturbance in it include Baltic rush (Juncus balticus), ticklegrass (Agrostis scabra), fowl bluegrass (Poa palustris), Kentucky bluegrass (P. pratensis), prairie sagewort (Artemisia ludoviciana), and common silverweed (Potentilla anserina). An unusual condition with extremely high cover of sowthistle (Sonchus spp.) was also found in the Lake Creek valley.

Carex praegracilis is common in small, intricate mosaic patterns across sandy flats affected by the elevated water levels on Lake Creek, where Juncus balticus, Elymus trachycaulus, and Muhlenbergia richardsonis are part of the mosaic and the numbers of associated species are low. The "natural" vegetation of these settings is unknown.

\section{Distichli sspicata Intermittently Flooded H erbaceous Vegetation}

Saltgrass I ntermittently Flooded H erbaceous Vegetation

\section{Global rank: G5; State rank: S4}

Summary: The saltgrass intermittently flooded plant association is found in the glaciated northern Great Plains at small bands and patches associated with alkali basins, and at isolated alkaline seeps. It is also found at the base of badlands erosional sideslopes, in outwash, and in alluvial fan settings of the unglaciated and thinly-glaciated northern Great Plains. The soils are moderately to strongly saline, medium- to fine-textured, and poorly developed but moderately deep to deep (Hansen 1995, USFS 1992). The water table is high for at least part of the year and salt encrustations are often present on the surface. This community has low species diversity and is dominated by salttolerant graminoids. Total vegetation cover is sparse to moderate and bareground is common. The dominant species, Distichlis spicata, comprises 5$55 \%$ canopy cover. Species diversity is low. Other salt-tolerant species often present include Nuttall's alkaligrass (Puccinellia nuttalliana), alkali muhly (Muhlenbergia asperifolia), sea blite (Suaeda calceoliformis) and Nevada bulrush (Scirpus nevadensis); the latter three particularly in glaciated settings. Common forbs include white- 
prairie aster (Aster falcatus), slimleaf goosefoot (Chenopodium leptophyllum), gumweed (Grindelia squarrosa), slender plantain (Plantago elongata), indian-wheat (Plantago patagonica), and red glasswort or samphire (Salicornia rubra).

In Sheridan County, this type is consistently part of alkali lake landscapes on soils mapped as ustifluvents, and sometimes present in saline upwellings along the Big Muddy Creek valley on Nobe clay.

\section{Eleocharis palustris $\mathrm{H}$ erbaceous Vegetation}

\section{Common Spike-rush H erbaceous Vegetation Global rank: G5; State rank: S5}

Summary: The common spike-rush plant association occurs in slighty to moderately brackish wetlands, including prairie potholes and riparian settings, that are seasonally flooded. It is wellrepresented in the central and northern Great Plains, possibly extending into the Southwest and the Pacific Northwest states, and is a major type at low elevations throughout Montana (Hansen 1995). Species often present include needle spike-rush (Eleocharis acicularis; often submerged early in growing season), curly dock (Rumex crispus), and foxtail barley (Hordeum jubatum).

In Sheridan County, it is found in Missouri Coteau prairie potholes, and on tributaries of the Big Muddy Creek in standing water of riparian channels that become series of small pools by midsummer, often surrounded by common threesquare (Scirpus pungens) zones.

\section{Eleocharisquinqueflora $M$ arl Fen $H$ erbaceous Vegetation}

Few-flowered Spike-rush M arl Fen $\mathrm{H}$ erbaceous $V$ egetation

Global rank: G 1?; State rank: S1

Summary: The few-flowered spike-rush/ chara marl fen herbaceous vegetation is a patterned, rich fen plant association of the northern Great Plains found in localized areas where mineral-rich groundwater flow emerges at the ground's surface. The dominant or co-dominant species include
Eleocharis quinqueflora, with shallow, open-water pools dominated by Chara spp., a calciphilic macroalgae that contributes to calcium carbonate precipitation. This plant association is documented from North and South Dakota, and northeastern Montana, and may be present in adjoining provinces.

Vegetation: This plant association is characterized by fine-textured short-statured vegetation including few-flowered spike-rush (Eleocharis quinueflora), beakrush (Rynchospora capillacea; not known from Montana), slender muhly (Muhlenbergia filiformis), mat muhly (M. richardsonis), Kalm's lobelia (Lobelia kalmii), and grass-of-parnassus (Parnassis spp.) (Leoschke 1997). The open marl pools consistently host Chara, a calciphilic macroalgae that contributes to calcium carbonate precipitation. A number of abundant or diagnostic mosses are present in both mound and pool microhabitats. Species that were restricted to or reached their highest densities in these settings as they occurred in Sheridan County include: rush aster (Aster junciformis), green sedge (Carex viridula), Kalm's lobelia (Lobelia kalmii), Muhlenbergia filiformis, Torrey's rush (Juncus torreyi), and marsh arrow-grass (Triglochin palustre).

Environment: This plant association is found where mineral-rich groundwater flow emerges from porous, glacial till. These areas remain saturated throughout the growing season, permitting the development of organic peat enriched in calcium carbonate. The intact patterns form parallel moundpool (string-flark) microhabitats, with the mounds dominated or co-dominated by Eleocharis quinqueflora, and other short-statured graminoids, and the shallow, open-water pools dominated by Chara spp. In central and northwestern North Dakota, these associations are found on slopes bordering wetlands and along river valley slopes. These wetland areas remain saturated throughout the growing season, permitting the development of organic peat. These associations form parallel mounds and shallow, interconnected pools, all of which are composed of marl, a mix of calcium carbonate, organic matter, and other minerals (Duxbury 1987, Chadde et al. 1998).

Comments: Under heavy grazing, Triglochin palustre and T. maritimum increase and under extreme cases dominate, sometimes in combination with more robust plants like water sedge (Carex 
aquatilis) and common threesquare (Scirpus pungens). Then, the parallel mound pattern is changed into irregularly trampled hummocks, the pools become eutrophied, and emergent vegetation becomes established in them.

This plant association requires stable groundwater discharge, and central pivot irrigation has been installed near or adjacent to many of the sites since the 1980s.

This is the habitat of Lobelia kalmii, which appears to be restricted to Eleocharis quinqueflora plant associations throughout its range in Montana.

\section{H ordeum jubatum $\mathbf{H}$ erbaceous Vegetation Foxtail Barley $\mathrm{H}$ erbaceous Vegetation Global rank: G4; State rank: S4}

Summary: The foxtail barley herbaceous vegetation is a seasonally flooded subsaline wetland type (Thompson 1994), and a drawdown phase of seasonal and semipermanent brackish and subsaline marshes. It was noted as common among the temporary ponds of the Missouri Coteau area of Sheridan County (Lesica 1987b), a commonness that was not observed in this study, and which may have reflected the drought conditions of the time that the earlier work was conducted.

Comments: Hordeum jubatum is associated with Distichilis spicata and Pascopyrum smithii, and becomes abundant to the point of appearing dominant in these associations when they are under intense grazing. It has also been noted that Hordeum jubatum can dominate on less saline sites (Redmann 1972) than Distichilis spicata, and is also a draw-down phase of semi-permanent brackish wetlands under natural conditions (Stewart and Kantrud 1971).

\section{Paniaum vi rgatum - Muhlenbergia richardsonis - (Schi zachyri um scopari um) $H$ erbaceous Vegetation \\ Switchgrass - M at M uhly - (Little Bluestem) $\mathrm{H}$ erbaceous V egetation \\ Global rank: G?; State rank: S?}

Summary: The switchgrass - mat muhly - (little bluestem) herbaceous vegetation is a highly productive grassland associated with subirrigated conditions and groundwater discharge as found in glaciated valleybottom settings, sometimes associated with springs, seeps, and spring-fed streams. It is presented in this study as a "new" plant association, not recognized on regional plant association lists to date.

All of the Sheridan County examples are part of outwash channels, as found in the Medicine Lake and Missouri Coteau areas. They may be found in valleybottoms, small isolated "pockets" of habitat above large alkali wetlands, or meander borders.

Vegetation: The plant association has $100+\%$ vegetation cover dominated by tall- or mid-height grasses. There is typically codominance of Panicum virgatum or Muhlenbergia richardsonis, with or without major cover contributions of Schizachyrium scoparium. Slender wheatgrass (Elymus trachycaulus) and tufted hairgrass (Deschampsia cespitosa) are often present. Forbs commonly found in this setting include dotted blazing-star (Liatris ligustistylis), meadow hawksbeard (Crepis runcinata var. glauca), and lance-leaf goldenweed (Haplopappus lanceolatus).

Environment: This plant association is associated with glacial outwash valleys and occasionally found on the seeps of closed-basin alkali lakes scattered within them. The groundwater is close to the surface through most of the growing season, and the fine-textured soils are moderately alkaline and poorly-drained. Along the Lake Creek valley, they are mapped as McKenzie silty clay loams.

Comments: This plant association was historically used for hayland where it covered any extent. But with the trend in larger machinery and the continued reduction in area, most of the current examples are in rangeland. Late-season grazing seems to have the least affect on it.

Kentucky Bluegrass (Poa pratensis) is often 
present in trace amounts, increasing under heavy grazing. Early-season grazing can also cause formation of hummocks and drying of the habitat under some environmental conditions. Litter accumulation is high in this plant association type, and may suppress forb numbers.

This is thought to be the primary habitat for palespiked lobelia (Lobelia spicata) and possibly for northern blue-eyed grass (Sisyrinchium septentrionale).

\section{Pascopyrum smithii H erbaceous Vegetation}

Western Wheatgrass H erbaceous V egetation Global rank: G 3G5Q ; State rank: S4

Summary: The western wheatgrass herbaceous vegetation is a wetland type, even though the species is also dominant or codominant in many upland plant associations. This plant association is found in both temporarily and seasonally flooded wetlands, and is the most widespread seasonally flooded wetland plant association in parts of northeastern Montana, as documented in Thompson (1994). It occurs on fine-textured soils. Cover of western wheatgrass is high; typically greater than $70 \%$, and species diversity is low. Characteristic forbs include gumweed (Grindelia squarrosa), wild lettuce (Lactuca canadensis), horseweed (Conyza canadensis) and nodding stickseed (Hackelia deflexa). It is considered to be a minor component of the mixed grass prairie region in Saskatchewan (Riparian and Wetland Research Program 2000).

It is part of riverine systems in Sheridan County, and is common on clay substrates in poorly-drained settings along the Big Muddy Creek and tributaries.

Comments: Under disturbance, exotic species increase, including Japanese brome (Bromus japonicus), curly dock (Rumex crispus), red sage (Kochia scoparia), and Loesel's tumblemustard (Sisymbrium loeselii).

\section{Polygonum amphi bium $\mathrm{H}$ erbaceous Vegetation}

Water Smartweed H erbaceous Vegetation G lobal rank: G3?; State rank: S?

Summary: The water smartweed herbaceous vegetation is found in seasonally inundated, slightly brackish wetlands where the basin has been plowed in the past (Stewart and Kantrud 1971). It is also found at zones around reservoirs, ponds, and other wet areas (Hansen 1995). Water smartweed is a widespread wetland species, but does not appear to assume dominance in the absence of perturbations. This plant association is scattered across northeastern areas of Sheridan County in settings where part or all of the bottom of the wetland basin had been plowed at some time in the past.

\section{Potamogeton pecti natus- Myri ophyllum spicatum H erbaceous Vegetation \\ Sago Pondweed - Common Water-milfoil $\mathrm{H}$ erbaceous V egetation \\ Global rank: G3G4; State rank: S1Q}

Summary: Sago pondweed - common watermilfoil herbaceous vegetation occurs in permanently innundated zones of glacial ponds in gentle, rolling, glacial terrain. It has been reported from north-central Montana (Lescia 19X), with similar or closely-related assocations in western Montana, North Dakota, and Saskatchewan; also reported from California. This type occurs in permanent ponds with mildly brackish water less than about 1 $\mathrm{m}$ deep. Submerged species dominate the stands with low canopy cover (50\%). Potamogeton pectinatus is always present and usually accompanied by Myriophyllum spicatum.In addition, water buttercup (Ranunculus aquatilis), common bladderwort (Utricularia vulgaris), and Richardson's pondweed (Potamogeton richardsonii) are commonly associated species in some ponds

In Sheridan County, submerged vegetation dominated or co-dominated by sago pondweed is in the Missouri Coteau wetlands as well as the channel of the Big Muddy Creek.

Comments: Sago pondweed herbaceous vegetation comprises the most extensive submerged vegetation in both riparian and lacustrine wetland settings. 
The species has been characterized as the single most important food for waterfowl elsewhere in the Missouri Coteau because of its abundance, and the nutritional value of both the seeds and the tubers (Metcalf 1931).

\section{Puccinellia nuttalliana H erbaceous Vegetation}

\author{
$\mathrm{N}$ uttall's A lkaligrass $\mathrm{H}$ erbaceous Vegetation \\ Global rank: G3?; State rank: S?
}

Summary: The Nuttall's alkaligrass herbaceous vegetation requires seasonally saturated saline soils as found in wet meadows, saline seeps, and the margins and flats associated with alkali lakes. It is found on glaciated and unglaciated areas of the Great Plains and intermountain region, extending from Alberta and Saskatchewan to Nebraska, and west through the intermountain region to Utah and California. The settings are moisture-collecting positions that are temporarily or seasonally inundated, maintaining moist soil conditions throughout most growing seasons, though drying out at the surface. Species diversity is low and variously includes saltgrass (Distichilis spiata), foxtail barley (Hordeum jubatum), alkali bluegrass (Poa juncifolia; treated by some taxonomists as synonymous with Poa secunda), red goosefoot (Chenopodium album), sea blite (Suaeda calciformis), and amaranth (Amaranthus spp.) In eastern Alberta it is described as a saline emergent marsh or hypersaline dry meadow (Wallis 1990), and it is an incidental component of the mixed grass prairie region in Saskatchewan (Riparian and Wetland Research Program 2000).

In Sheridan County, it is found as part of alkali lake systems and in localized low-lying settings with salt accumulation, including the Big Muddy Creek valley and tributaries.

Comments: Nuttall's alkaligrass is generally considered a decreaser under livestock grazing (Smith 1976). In Sheridan County, it was found in conditions ranging from idle to trampled.

\section{R uppia maritima G reat Plains \\ $H$ erbaceous Vegetation}

Wigeon-grass G reat Plains H erbaceous Vegetation

Global rank: G?; State rank: S?

Summary: Wigeon grass plant associations are a submerged vegetation restricted to saline lakes and some of the associated subsaline wetlands, with a conductivity normally over $15,000 \mathrm{micromhos} / \mathrm{cm} 3$ (Stewart and Kantrud 1971). Many of the settings are aptly called hypersaline, with higher salinity than the ocean. They occur in the glaciated plains regions of Alberta, Saskatchewan, Montana and North Dakota, typically in closed-basin watersheds, often part of glacial outwash channels. More information is needed on cover values and distribution of the submerged vegetation as affected by water depth, water chemistry, and wave action.

In Sheridan County, the wigeon-grass plant association was found along the large outwash channels toward the eastern end of the county in brackish wetlands.

Comments: The plant association has direct wildlife value in that wigeon-grass has been characterized as one of three major foods for waterfowl elsewhere in the Missouri Coteau (Metcalf 1931).

\section{Salicornia rubra $\mathbf{H}$ erbaceous Vegetation Red Glasswort (Samphire) H erbaceous Vegetation Global rank: G3G 4; State rank: S2?}

Summary: The red glasswort herbaceous vegetation is found on the shores of saline semipermanent ponds and lakes (Stewart and Kantrud 1971) and associated alkali flats during the dry or draw-down phase. It is mainly in the prairie pothole region, with reports of similar associations in Colorado and Nevada. Soils are subsaline or saline, gleyed, with generally fine textures ranging from silt loam to clay (Looman 1981). It occupies the seasonal drawdown areas that are generally too wet for saltgrass (Distichilis spicata). Vegetation is typically sparse and patchy, with Salicornia rubra sometimes making up all of the vegetation within these areas, with or without Nuttall's alkaligrass 
(Puccinellia nuttalliana), foxtail barley (Hordeum jubatum), common arrow-grass (Triglochin maritimum), red goosefoot (Chenopodium rubrum), and sea blite (Suaeda calceoliformis). This plant association is among the few dominated by an annual species; making major shifts in cover between drought and high-water conditions, but generally a persisting feature.

In Sheridan County, there were vast expanses of red glasswort south of Salt Lake under the somewhat dry conditions of 1998 that were innundated and part of a sparse alkali bulrush (Scirpus maritimus) wetland under closer-to-normal water levels in 1999. It is recognized in this vegetation classification primarily as a persisting component of the alkali lake system, rather than as an ephemeral drought-response phenomenon.

\section{Scirpusacutus $\mathrm{H}$ erbaceous Vegetation} $\mathrm{H}$ ardstem Bulrush $\mathrm{H}$ erbaceous Vegetation Global rank: G5; State rank: S5

Summary: The hardstem bulrush herbaceous vegetation is among the most common plant associations of semipermanently flooded wetlands in parts of northeastern Montana (Thompson 1994) and at low elevations throughout Montana (Hansen et al. 1995). It dominates in wetlands with water chemistry conditions that are mildly- to moderately-brackish (Stewart and Kantrud 1972). The tall emergent vegetation is $1-2 \mathrm{~m}$ tall, and varies greatly in density with annual changes in water levels and muskrat activity.

In Sheridan County, it is particularly extensive around Lake Creek, including areas of Medicine Lake NWR with artificially maintained water levels. It is also dominant in many of the larger prairie potholes.

\section{Scirpusmariti musH erbaceous Vegetation}

\section{Alkali Bulrush $\mathrm{H}$ erbaceous Vegetation Global rank: G4; State rank: S4}

Summary: Plant associations dominated by alkali bulrush are common in brackish and subsaline semipermanently flooded wetlands, and to a lesser extent, perennial streams. They are characteristic of saline emergent marsh in the provinces (Looman 1981, Wallis 1990) and have been recognized elsewhere in northeastern Montana (Thompson 1994). The alkali bulrush plant association is often an emergent band around open water with submerged vegetation of Potamogeton pectinatus or Ruppia maritima. It also occurs as zonal vegetation around other plant associations. The cover of alkali bulrush may almost disappear in openwater conditions of wet years, or be replaced by red glasswort (Salicornia rubra) in drought years.

In Sheridan County, it is mainly in the Missouri Coteau prairie pothole area where there are glacial meltwater channels. Many but not all wetlands dominated by alkali bulrush are part of alkali lake landscapes.

Comments: Alkali bulrush has been characterized as one of three major foods for waterfowl elsewhere in the Missouri Coteau (Metcalf 1931).

\section{Sa rpus pungens $\mathrm{H}$ erbaceous Vegetation Common Threesquare $\mathrm{H}$ erbaceous Vegetation \\ Global rank: G3G 4; State rank: S3}

Summary: The common threesquare herbaceous vegetation is found along low-gradient, usually perennial streams and around the margins of semipermanently-flooded ponds and marshes in the Intermountain Basin and the western and northern Great Plains (Hansen et al. 1995, Jones and Walford 1995, Stewart and Kantrud 1972). Characteristically, it is a "zonal" vegetation restricted to bands rather than occurring throughout basins of suitable depth. It is typically in shallow, brackish water, in settings where salt often precipitates when water levels drop. Scirpus pungens dominates the herbaceous vegetation layer, which is 1 foot to 2 feet tall; other graminoid species may be present are alkali cordgrass (Spartina gracilis), foxtail barley (Hordeum jubatum), western wheatgrass (Pascopyrum smithii), and common spike-rush (Eleocharis palustris). Forb species are few. Note: This includes plant associations previously referred to as having dominance by Scirpus americanus Olney (Stewart and Kantrud 1972, and Looman 1981) citing the taxonomic treatment that is recognized as a synonym with $S$. pungens (Great Plains Flora Committee 1986). 
The sharp bulrush plant association occurs in a wide variety of Sheridan County settings, though it is nowhere extensive. It is an outer zone around many shallow and deep brackish wetlands, and often the only emergent vegetation on alkali lakes, in broken shoreline segments marking groundwater discharge. It is similarly referred to as saline emergent marsh in eastern Alberta (Wallis 1990). It is also in seasonally or semipermanently-flowing tributaries of the Big Muddy Creek.

\section{Scolochloa festucacea H erbaceous Vegetation \\ Sprangletop (Whitetop) H erbaceous \\ Global rank: G4G 5; State rank: S?}

Summary: The sprangletop herbaceous vegetation is found in the northern Great Plains, including North Dakota, Montana, Manitoba and Saskatchewan. Stands occur in seasonally- and semipermanently flooded sites with water 0.3-1 m deep. Stands dominated by Scolochloa festucacea are usually slightly to moderately brackish (Looman 1981, 1982, Stewart and Kantrud 1972). Graminoids 1.0-2.0 m tall dominate the moderately dense to dense vegetation cover. Scolochloa festucacea is the single most abundant species and may occur in almost monotypic stands. Other species sometimes present include awned sedge (Carex atherodes), common spike-rush (Eleocharis palustris), American mannagrass (Glyceria grandis), hemlock water-parsley (Sium suave), star duckweed (Lemna trisulcata) and common bladderwort (Utricularia vulgaris).

The sloughs dominated by sprangletop were once exceedingly abundant in northern North Dakota (Metcalf 1931), and Metcalf called these same sloughs "mallard sloughs." The seeds of sprangletop were sought by waterfowl, and the sloughs were most frequented by them. Few examples of this type have been noted in Sheridan County, restricted to the Missouri Coteau prairie potholes area.

\section{Triglochin maritimum $\mathrm{H}$ erbaceous Vegetation \\ Common Arrow-grass H erbaceous Vegetation \\ Global rank: GU ; State rank: S?}

Summary: Common arrow-grass herbaceous vegetation is provisionally described as a saline wetland vegetation that may occur in the absence of disturbance, but definitely occurs under heavy livestock grazing. It is present on fine-textured soils of saline and subsaline lakes, seeps, and associated inlets. Vegetation is dominated by common arrowgrass often intermixed with Nuttall's alkaligrass (Puccinnellia nuttalliana) and alkali bulrush (Scirpus maritimus). It corresponds with the saline emergent marsh of eastern Alberta (Wallis 1990).

In Sheridan County, the most extensive stand of this plant association was found on a large wetland basin that no longer holds water in the summer. It has not routinely been considered a drawdown species, and the setting may represent a site where the water table has been lowered. It occurs naturally in narrow bands as part of the alkali lake system, sometimes forming $100 \%$ canopy cover along inlets. It was also dominant at alkaline seeps in the Big Muddy valley under heavy grazing pressure.

\section{Typha lati folia Western H erbaceous Vegetation \\ Common Cattail Western $\mathrm{H}$ erbaceous Vegetation \\ Global rank: G5; State rank: S5}

Summary: The common cattail western herbaceous association is found throughout the Northern Great Plains. Stands occur in semipermanent wetlands, as well as in stock ponds, and seepy drainages. The vegetation is dominated by relatively pure stands of Typha spp., including Typha latifolia, lesser cattail (Typha angustifolia), or hybrids between the two. Dominance by $T$. latifolia occurs in fresh and slightly brackish conditions. Dominance by T. angustifolia occurs under more alkaline conditions. The hybrid occurs in roadside ditches and borrow pits, as well as natural wetlands where the water levels vary greatly. The Typha latifolia wetland plant 
association is considered to be a major component of the mixed grass prairie region in Saskatchewan (Riparian and Wetland Research Program 2000)

The common cattail plant association is uncommon in Sheridan County. The only large stand noted was in nearby Homestead Lake NWR. A narrowleaf cattail thought to correspond with the hybrid is also present. Elsewhere in the county, a narrow-leaf cattail was noted in distinct bands around alkaline springs, thought to be T. angustifolia.

\section{Wetland Types U nder Review}

The following wetland plant associations were noted from no more than two plots or observations, and they do not mesh with plant associations described for immediately adjoining areas of the plains. They are treated as provisional, warranting expanded field investigation and further consideration.

\section{C alamagrostistricta $\mathrm{H}$ erbaceous Vegetation \\ $\mathrm{N}$ arrow-spiked Reedgrass $\mathrm{H}$ erbaceous Vegetation}

Summary: Narrow-spiked reedgrass is an occasional-to-common species in wet meadows of Sheridan County, and was a localized dominant on seeps along the Lake Creek Valley, with $90 \%$ cover of Calamagrostis stricta and 10\% cover of woolly sedge (Carex lanuginosa). It adjoined the Carex praegracilis plant association and the Eleocharis quinqueflora / Chara spp. plant association.

Further vegetation sampling is needed to determine whether this is a repeating plant association on the landscape.

\section{C arex aquati lis $\mathbf{G}$ reat Plains $\mathrm{H}$ erbaceous Vegetation \\ Water Sedge H erbaceous Vegetation}

Summary: The water sedge herbaceous vegetation of the Prairie Region is found in the northern Great
Plains. Stands typically occur in depressions or around ponds or lakes, although they may also develop adjacent to streams or rivers. The sites on which it develops are flooded for some time during the growing season in most years and have fresh or slightly saline water. The Canadian literature reports that this association is found mainly on mineral soils (Looman 1981, 1982), while the montane form of this association is found mainly on organic soils (Hansen et al. 1995) as were the limited examples found in Sheridan County. The vegetation is dominated by graminoids approximately 0.4 to $0.7 \mathrm{~m}$ tall. Carex spp. predominate, especially Carex aquatilis, as well as beaked sedge (Carex utriculata), lake sedge (Carex lacustris), and tussock sedge (Carex stricta) in parts of its range. Note: The latter two are uncommon in Montana, and the Carex utricultata is in the state as a montane wetland plant association. Other species found in this community include common spike-rush (Eleocharis palustris), bullrushes (Scirpus spp.), and cattails (Typha spp.) Typical forbs include rough bugleweed (Lycopus asper), western dock (Rumex occidentalis), and hemlock water-parsley (Sium suave).

In Sheridan County, this is a minor plant association that occurs as small patches where there is groundwater discharge or springs along wetland borders. Most places where it occurs are in outwash channels. It is found in small isolated pockets or outer margins of peatland habitat as the only peatland habitat, or enircling the few-flowered spike-rush plant association (Eleocharis quinqueflora).

\section{Elymustrachycaulus $\mathrm{H}$ erbaceous Vegetation \\ B earded Wheatgrass H erbaceous V egetation}

Summary: Thick-spike wheatgrass appeared to dominate a wet meadow area in the Lake Creek Valley, comprising app. 70\% cover. Other grasses that were respresented by more than $1 \%$ cover included Muhlenbergia richardsonis and Panicum virgatum. The most abundant forb was Helianthus nuttallii. Further vegetation sampling is needed to determine whether this is a repeating plant association on the landscape. 


\section{Elymustrachycaulus- D istichilis spicata $H$ erbaceous Vegetation \\ Bearded Wheatgrass - Saltgrass H erbaceous Vegetation}

Summary: The bearded wheatgrass - saltgrass Herbaceous Vegetation appears to be a wet meadow plant association associated with alkali lakes. It is restricted to soils with high clay content, frequently found on points or penninsulas along the shoreline. It lies within the broad zones mapped as saline ustifluvent soil.

\section{Elymustrachycaulus- Spartina gracilis $H$ erbaceous Vegetation}

\section{Bearded Wheatgrass - Alkali Cordgrass $\mathrm{H}$ erbaceous $V$ egetation}

Summary: An association dominated by bearded wheatgrass and alkali cordgrass was documented in temporarily inundated wet meadow on clay soils among a series of wetlands associated with an alkali lake. Further vegetation sampling is needed to determine whether this is a repeating plant association on the landscape.

\section{Panicum vi rgatum - Sparti na pecti nata $H$ erbaceous Vegetation \\ Switchgrass - Prairie Cordgrass H erbaceous Vegetation}

Summary: An association codominated by switchgrass and prairie cordgrass was documented in a spring-fed tributary of the Big Muddy Creek. The latter generally occupies wetter habitat, and it is possible that their co-dominance is more appropriately treated as an ecotone between separate switchgrass and prairie cordgrass plant associations.

\section{Scirpusnevadens s $\mathrm{H}$ erbaceous Vegetation}

$\mathrm{N}$ evada Bulrush $\mathrm{H}$ erbaceous Vegetation

Summary: The Nevada Bulrush herbaceous vegetation is characterized as typical of sandy saline shores of eastern Alberta (Wallis 1990). It is at best a very broad and long vegetation zone like Scirpus pungens. The largest area of it was less than 1 acre, noted on a saline seep along the Lake Creek Valley, and noted elsewhere on Medicine Lake NWR, Brush Lake area, and Goose Lake.

\section{Spartina gracilis $\mathrm{H}$ erbaceous Vegetation Alkali Cordgrass $\mathrm{H}$ erbaceous Vegetation}

Summary: Alkali cordgrass is dominant in narrow zones and patches with high cover values. It formed discrete bands around alkaline springs, and included such species as foxtail barley (Hordeum jubatum), rush aster (Aster junciformis) and seamilkwort (Glaux maritima). Further vegetation sampling is needed to evaluate this as a minor plant association.

\section{Sparti na pecti nata $\mathrm{H}$ erbaceous Vegetation \\ Prairie Cordgrass $\mathrm{H}$ erbaceous Vegetation}

Summary: The prairie cordgrass herbaceous vegetation is typically found in slightly or moderately brackish wetlands that are temporarily or seasonally inundated. It is treated by Hansen et al. (1995) as similar with regards to environment and management as plant communities dominated by Spartina gracilis, and the collective cordgrass associations are characterized as extending into saline conditions. Most plant association descriptions are from unglaciated portions of the Great Plains, from drainage bottoms where the soil is wet for at least part of the growing season, or in poorly drained depressions within floodplains of major rivers. In the glaciated plains, it is often associated with groundwater discharge in riverine systems forming seasonally-inundated wet meadow; and occasionally in temporarily or seasonally flooded wetlands. Further vegetation sampling and review of existing plant associations dominated by Spartina pectinata is needed for classification purposes.

In Sheridan County, it was found in an upper reach of the Lake Creek valley where Spartina pectinata was sole dominant.

Comments: It is a highly productive grassland found in a narrow range of conditions, readily altered by grazing and any on-site/off-site changes to water conditions. 


\section{Shrubland Types}

Shrublands are widely scattered in Sheridan County but are not extensive. They are variously referred to as thickets, scrub, and steppe. We have included grassland and sparse vegetation types here in which shrubs have a strong presence but have less than $25 \%$ canopy cover. Stands with this structure and composition have a unique designation in the classification at the formation level.

\section{A rtemisia cana / Pascopyrum smithi i Shrubland \\ Silver sagebrush - Western Wheatgrass Shrubland \\ Global rank: G4; State rank: S4}

\begin{abstract}
Summary: The silver sagebrush - western wheatgrass shrubland is found mainly in the western Great Plains on flat alluvial deposits on floodplains, terraces or benches, and alluvial fans. The soils are moderately deep to deep and either silt loam, clay loam, or sandy loam. Flooding may occur periodically and this tends to retard soil development. This community is dominated by a combination of shrubs and graminoids. The total vegetation cover is moderate. The tallest and most conspicuous stratum in this community is a shrub layer that is usually $0.6-1.2 \mathrm{~m}$. dominated by Artemisia cana. Grass cover is dominated by Pascopyrum smithii and typical forbs of this community are yarrow (Achillea millefolium), scarlet gaura (Gaura coccinea), red globe-mallow (Sphaeralcea coccinea), and blue lettuce (Lactuca pulchella).
\end{abstract}

This plant association is uncommon in glaciated landscapes, and was only noted in Sheridan County along an isolated area of Wolf Creek, and Beaver Creek in the Big Muddy headwaters. While this plant association is not restricted to riparian settings, those observed fit the wetland characterization for it as presented in Hansen et al. (1995). In Sheridan County, Artemisia cana comprised canopy cover which was usually less than $25 \%$, more appropriately referred to as shrub herbaceous vegetation.

\section{Elaeagnus commutata Shrubland \\ Silverberry Shrubland \\ Global rank: G2Q ; State rank: S2?}

Summary: Silverberry shrubland is reported in northern Montana east of the Continental Divide, and generally classified as temporarily flooded. A related association with an understory of Pascopyrum smithii is reported from Hill County, provisionally representing the "typical" floodplain type. The validity, composition, and status of silverberry shrublands needs documentation and further evaluation, including more information on the floodplain stands from Saskatchewan, and more information on upland stands to the east.

Small pockets of Elaeagnus commutata plant assocation with a needle-and-thread (Stipa comata) understory are present in the Medicine Lake sandhills of Sheridan County, with shrub canopy cover of over $10 \%$ and grass canopy cover over $70 \%$. It is not flooded, but the water table is within rooting zone. The species is also common on a few of the north-facing northern porcupinegrass (Stipa curtiseta) slopes in the county that have high levels of litter accumulation, and may be on the increase in such settings.

\section{Eriogonum pauciflorum - G utierrezia sarothrae Badlands Sparse Vegetation}

Few-flowered Wild Buckwheat - Broom Snakeweed Badlands Sparse Vegetation

Global rank: G?; State rank: S?

Summary: The few-flowered wild buckwheat broom snakeweed Badlands sparse vegetation represents an outcrop or scabland feature of the northern Great Plains in Montana, North Dakota and South Dakota. It occurs on unconsolidated or poorly-consolidated sedimentary formations that are easily eroded, including siltstones, mudstones, and shales. Slopes that become stabilized revegetate to grassland with succession.

This badlands plant association type rarely exceeds $10 \%$ vegetative cover and is often less than $5 \%$, hence it is technically placed in a sparse vegetation classification category rather than a grassland plant association. On level terrain, the vegetation is relatively evenly distributed, but on steeper slopes 
and cliffs the vegetation may grow in patches and in rows or seams. Plant species that are nearly always present include few-flowered buckwheat (Eriogonum pauciflorum), broom snakeweed (Gutierrezia sarothrae), plains pricklypear (Opuntia polyacantha), common rabbitbrush (Chrysothamnus nauseosus), silverscale (Atriplex argentea), a cryptantha (Cryptantha thyrsifolia, a characteristic plant at least in South Dakota), and the forb gumweed (Grindelia squarrosa). In Sheridan County, it is restricted to the most exposed and rugged breaks of the Big Muddy valley and major tributaries cutting into bedrock. It encompasses the "Chrysothamnus nauseosus / Eriogonum pauciflorum plant association type" previously described in southeastern Montana where sparse Badlands vegetation is more common (Cooper and Heidel 1998).

Comments: Few-flowered wild buckwheat is considered to be a "sub-shrub", thus, the plant association is included among shrub-dominated plant associations.

\section{Prunusvirginiana Shrubland Common Chokecherry Shrubland Global rank: G4Q ; State rank: S4}

Summary: This was provisionally treated as two separate sandhills plant associations, the Prunus virginiana/ Stipa comata Shrubland and the Prunus virginiana/ Agropyron caninum (Elymus

trachycaulus) Shrubland by Lesica (1987a). In this study, all occurrences noted were smaller than the sample plot size, having high shrub cover and very low understory cover. Heavy browsing and levels of disease were noted. More complete characterization of this as vegetation is needed. It is generally considered a successional phase of the green ash- or box elder-dominated Woodlands (Fraxinus pensylvanica or Acer negundo Woodland) in eastern Montana (Hansen et al. 1995).

Prunus virginiana has a deep root system that can reach the sandhills water table. Elsewhere in northeastern Montana, it forms a riparian corridor plant association, classified as a seasonally flooded wetland (Thompson 1994).

\section{Sarcobatusvermiculatus/ Pascopyrum smithi i Shrub H erbaceous Vegetation \\ Black G reasewood / Western Wheatgrass Shrub H erbaceous Vegetation Global rank: G4; State rank: S4}

Summary: The black greasewood / western wheatgrass shrub herbaceous vegetation is found in the northwestern Great Plains on flat to gently sloping alluvial fans, terraces, lakebeds, and floodplains. The soil is usually deep clay, silty clay, sandy clay, or loam although coarse soils are possible. They are saline or alkaline but salt crusts on the surface are absent. Parent material is usually alluvium. This community has moderate to dense vegetation cover. Medium tall (0.5-1.5 m) shrubs are scattered throughout with a total shrub canopy of 5-25 percent, dominated by Sarcobatus vermiculatus. Few forbs are found in this community, and yarrow (Achillea millefolium) and plains pricklypear (Opuntia polyacantha) are the only species with high constancy.

In Sheridan County, it is present in salt-affected flats with Nobe clay substrate along the Big Muddy Creek, corresponding with the saline lowland range site. It is a major type elsewhere in eastern and central Montana (Hansen et al. 1995).

\section{Shepherdia argente Shrubland Buffaloberry Shrubland Global rank: G3G 4; State rank: S3?}

Summary: The buffaloberry shrubland is a mesic shrubland plant association found in the northern Great Plains from North Dakota and Saskatchewan to Colorado on stream terraces, rolling uplands, and badlands. It occurs where moisture is more plentiful than on the surrounding landscape, such as in swales, ravines, near streams, and on northwest- to east-facing slopes. This plant association is dominated by a moderate to dense canopy of medium-tall shrubs. The most abundant of these, Shepherdia argentea, is typically $1.5-3 \mathrm{~m}$ tall. Other common shrub species are common chokecherry (Prunus virginiana), skunkbush sumac (Rhus aromatica), Wood's rose (Rosa woodsii), and western snowberry (Symphoricarpos occidentalis). Graminoids and forbs may have only half the coverage of the shrub layer. Graminoids 
include western wheatgrass (Pascopyrum smithi), and invasion by Kentucky bluegrass (Poa pratensis). Common forbs are yarrow (Achillea millefolium), prairie sagewort (Artemisia ludoviciana), and common pellitory (Parietaria pennsylvanica).

The Shepherdia argentea plant association is not common in Sheridan County. Well-developed and extensive stands were noted along a deeply-incised segment of Eagle Creek in the Big Muddy headwaters. Elsewhere in its distribution, most stands to the south and east along the Missouri River are on sheltered upland prairie slopes, while most of the stands to the west are similarly in riparian settings, and classified as seasonally flooded wetland (e.g., Thompson 1994).

\section{Symphori carpos occi dentali s Shrubland Western Snowberry Shrubland G lobal rank: G 4G 5; State rank: S4S5}

Summary: The western snowberry shrubland is found in mesic depressions, swales, ravines and floodplains, typically surrounded by upland grassland associations. The soils are silts and loams. This type has a shrub layer (app. $80 \mathrm{~cm}$ tall) with high canopy cover, sometimes with welldeveloped graminoid- and forb-layers.

Symphoricarpos occidentalis is the predominant species in the shrub layer and at times forms almost monospecific stands. Rosa woodsii sometimes occurs interspersed with the Symphoricarpos occidentalis. Some stands are occasionally flooded whereas others are just very moist. Thus it tends to fall on both sides of the upland/wetland division. It has been classified in both temporarily and seasonally flooded wetland classes elsewhere in northeastern Montana, where it is among the most extensive of temporarily flooded wetland plant associations (Thompson 1994).

It is present throughout Sheridan County, including shallow prairie potholes, valley margins, and widely scattered elsewhere. It may accompany invasion of Kentucky bluegrass (Poa pratensis).

Comments: It corresponds with the Symphoricarpos occidentalis - Rosa woodsii association encroaching on sandhill flats and swales (Lesica 1987a). The same type was found in the Missouri Coteau prairie potholes in concave areas of lee slopes where snow accumulates (Lesica 1987b). It is also recognized as a major component of the mixed grass prairie region in Saskatchewan (Riparian and Wetland Research Program 2000).

\section{Shrubland Types U nder R eview}

\section{Juni perushorizontalis/ Elymuslanceolatus Shrubland \\ Creeping J uniper / Thick-spike Wheatgrass Shrubland}

Summary: Stands dominated by creeping juniper in association with thick-spike wheatgrass were documented in the Big Muddy headwaters on sheltered, north-facing slopes on silt loam derived from shale or other fine-textured parent material. This vegetation is reported elsewhere in northeastern Montana where it is more extensive, and it will be described separately in a separate document with larger sampling sets. Shrub cover is well over 50\%, with Juniperus horizontalis comprising almost all of the shrub cover and shrubby cinquefoil (Pentaphylloides floribunda) usually present. Species diversity is high, especially for forbs, and they include northern hedysarum (Hedysarum boreale), old man's whiskers (Geum triflorum), slender crazyweed (Oxytropis campestris), harebell (Campanula rotundifolia), pasqueflower (Anemone patens), and tufted fleabane (Erigeron cespitosus).

\section{Pentaphylloi desflori bunda / Sti pa curti sta Shrubland \\ Shrubby Cinquefoil / Northern Porcupinegrass Shrubland}

Summary: One stand dominated by shrubby cinquefoil in association with northern porcupinegrass was documented along the Big Muddy headwaters, on a sheltered, north-facing ravine slope. It has over $35 \%$ cover of shrubby 
cinquefoil, comprising almost all of the shrub cover, with shrub height approaching $3 \mathrm{ft}$. The cover of Carex inops at app. 20\% is almost as much as the graminoid cover of Stipa curtiseta. Forb diversity is high and northern bedstraw (Galium boreale) is especially abundant, approaching $20 \%$ cover, followed by northern hedysarum (Hedysarum boreale) and old man's whiskers (Geum triflorum). This provisional plant association does not appear to correspond with shrubby cinquefoil plant associations in the provinces, and is recommended for consideration as part of future vegetation ecology studies in northeastern Montana or southwestern Saskatchewan.

\section{Sarcobatusvermi culatus/ D istichlisspi cata - (Puccinnellia nuttalliana) Saline Shrub Sparse Vegetation}

Black G reasewood / Saltgrass - (N uttall's Alkaligrass) Saline Shrub Sparse Vegetation

Summary: Stands dominated by black greasewood in association with saltgrass or Nuttall's alkaligrass were recorded at several alkali lakes on their leeward shores. These lakes dry out periodically, and salt deposits from the lakebed are carried by the wind and redeposited onshore (Dodd and Coupland 1961). Soils are ustifluvents. Shrub cover is comprised entirely of Sarcobatus vermiculatus, app. 10-20\%, and usually stunted (less than $2 \mathrm{ft}$ tall). Graminoid cover is low at $10-40 \%$. Species diversity is low, and limited to halophytes like common arrow-grass (Triglochin maritimum).

\section{Woodland Types}

Woodlands are defined as "open stands of trees with crowns not usually touching, generally forming 25-60\% cover" (Grossman et al. 1998). They are present in small bands and pockets as dictated by the topography of ravines and escarpments. This topographic relief is conducive to moisture-concentration without salt accumulation, and provides a natural firebreak condition.

\section{Fraxinuspennsylvanica / Prunus virginiana Woodland Green Ash / Chokecherry Forest Global rank: G 3?; State rank: S2S3}

Summary: The Fraxinus pennsylvanica / Prunus virginiana Woodland represents islands of deciduous tree cover on the plains, known from the north- and central- Great Plains states. It is confined to sheltered topographic settings, often where moisture collects. The typical setting is found in ravines along the breaklands of major river courses, but also developed on escarpment slopes and a riparian climax vegetation following Populus deltoides in areas with sufficient moisture. Stands that occur in wooded ravines are often classified as temporarily flooded wetlands, and floodplain stands (not observed in Sheridan County) as seasonally flooded wetlands; both of which are documented and classified as such elsewhere in northeastern Montana (Thompson 1994). The herbaceous vegetation is typically dominated by Sprengel's sedge (Carex sprengelii). Other species present include serviceberry (Amelanchier alnifolia), western snowberry (Symphoricarpos occidentalis), Virginia wildrye (Elymus virginicus), false starry Solomen's-seal (Smilacina stellata), and Wood's rose (Rosa woodsii).

It is the only major woodland type in the county, of very limited extent in sheltered ravines along the Big Muddy Valley and tributaries, and widelyscattered on upland escarpment slopes on LambertZahill complex, 20-50\%. It represents the typical woody draw plant association of eastern Montana. The settings are relatively dry and the tree stature 
particularly short in the driest upland escarpments (less than $20 \mathrm{ft}$ ), though the stands have a welldeveloped woodland understory that includes species not present elsewhere in the county.

Comments: Woody draws are recognized for wildlife values (Severson and Boldt 1977), and are potentially vulnerable in Montana (Lesica 1987c). Kentucky bluegrass (Poa pratensis) increases with intensity of livestock use, and impedes tree seedling establishment. Some ravines along the Big Muddy valley are heavily invaded by leafy spurge (Euphorbia esula).

\section{Populustremuloi des/ Symphoricarposal bus Forest}

\section{Q uaking A spen / Common Snowberry Forest}

Global rank: G3?; State rank: S3?

Summary: The quaking aspen/ common snowberry forest is provisionally applied to small stands of quaking aspen in the most sheltered ravine segments that drain north into the Big Muddy Creek. They harbor boreal species present and not otherwise occurring in the county including red raspberry (Rubus idaeus), baneberry (Actaea rubra), fairybell (Disporum trachycarpum), and striped coral-root (Corallorhiza striata). The only stands that were visited had relatively tall $(>30 \mathrm{ft})$ tree canopy. Small, young groves of Populus tremuloides are also present in the sandhills, but did not have a native understory, and their growth and expansion was kept in check by grazing.

\section{Woodland Type U nder
Review}

\author{
Crataeguschrysocarpa Woodland \\ Yellow-fruit $\mathrm{H}$ awthorn Woodland
}

Summary: The yellow-fruit hawthorn woodland is a small plant association in the bottom of draws. It is reported from Saskatchewan (Riparian and Wetland Research Program 2000) and Alberta (Allen pers. comm.), including the Cypress Hills escarpment and other landforms with ravines. In Sheridan County it was found north of Raymond in side-draws along a deep glacial meltwater channel. The limited survey did not turn up examples with native species understory. 


\section{Appendix F. Characteristic plant associations of Sheridan County}

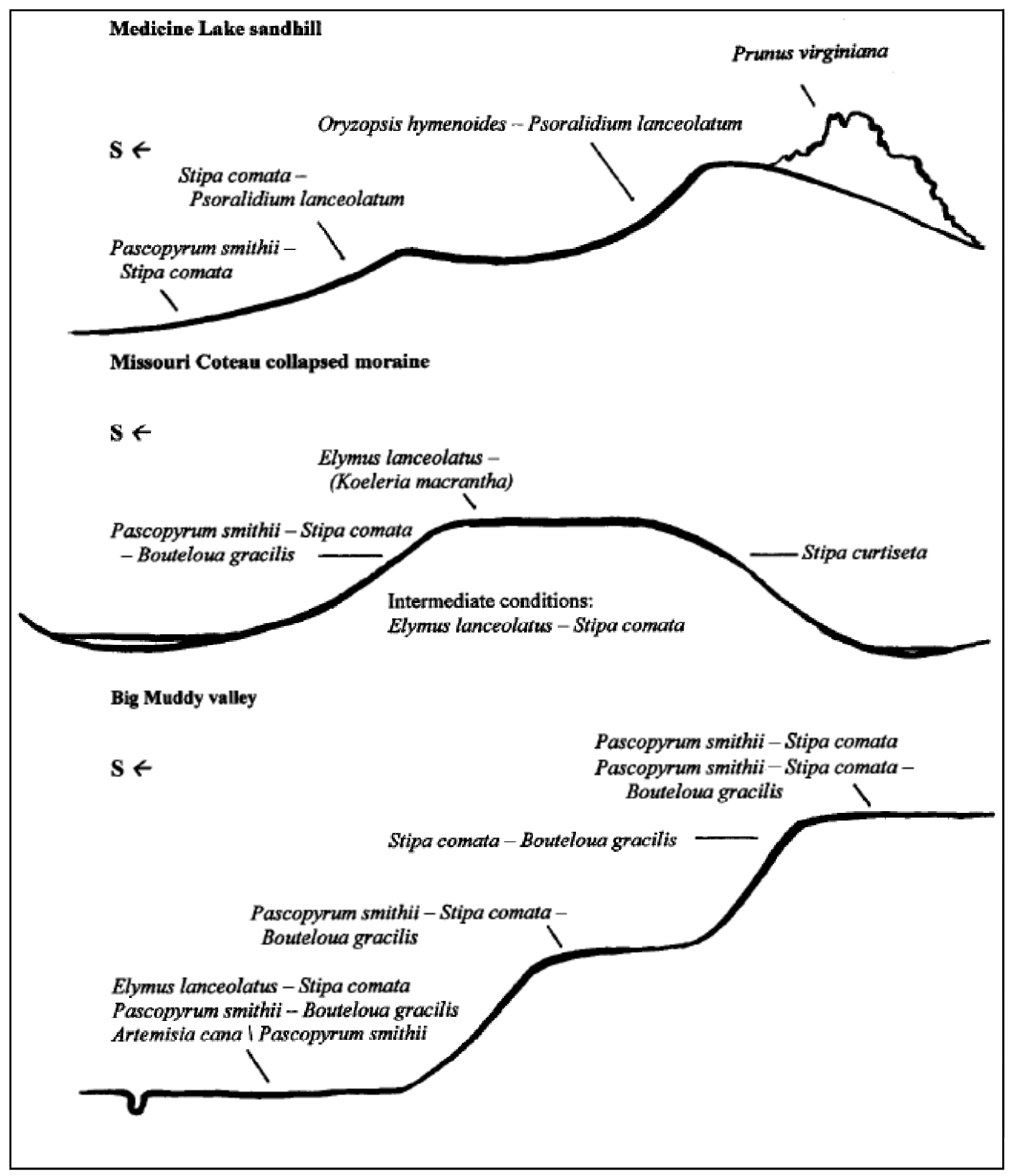




\section{Appendix G. -D raft - Range Sites, Ecological U nits* And Plant Associations** Of Sheridan County}

Northern Dark Brown Glaciated Plains (S3A); 10-14" precip. zone

\begin{tabular}{|c|c|c|c|}
\hline Range Site & Big Muddy Headwaters & Medicine Lake Area & Missouri Coteau Prairie Pothole \\
\hline Subirrigated & & $\begin{array}{l}\text { Panicum virgatum - Muhlenbergia richardsonis- } \\
\text { (Schizachyrium scoparium) } \\
\text { Carex praegracilis } \\
\text { Spartina pectinata. } \\
\end{array}$ & $\begin{array}{l}\text { Panicum virgatum - Muhlenbergia } \\
\text { richardsonis - (Schizachyrium scoparium) } \\
\text { Carex praegracilis }\end{array}$ \\
\hline $\begin{array}{l}\text { Saline } \\
\text { Lowland }\end{array}$ & $\begin{array}{l}\text { Sarcobatus vermiculatus/Pascopyrum } \\
\text { smithii } \\
\text { Distichilis spicata } \\
\text { Triglochin maritimum }\end{array}$ & $\begin{array}{l}\text { Distichilis spicata } \\
\text { Puccinnellia nuttalliana }\end{array}$ & $\begin{array}{l}\text { Sarcobatus vermiculatus/Distichilis spicata } \\
\text { Distichilis spicata } \\
\text { Pascopyrum smithii } \\
\text { Pascopyrum smithii-Hordeum jubatum } \\
\text { Elymus trachycaulus-Distichilis spicata }\end{array}$ \\
\hline
\end{tabular}

\begin{tabular}{|c|c|c|c|}
\hline Overflow & $\begin{array}{l}\text { Artemisia cana/Pascopyrum smithii } \\
\text { Pascopyrum smithii - Nasella viridula }\end{array}$ & & Pascopyrum smithii - Nasella viridula \\
\hline Sands & & $\begin{array}{l}\text { Oryzopsis hymenoides - Psoralidium } \\
\text { lanceolatum } \\
\text { Stipa comata-Psoralidium lanceolatum }\end{array}$ & \\
\hline Sandy & & $\begin{array}{l}\text { Calamovilfa longifolia - Stipa comata } \\
\text { Stipa comata - Bouteloua gracilis - Carex } \\
\text { filifolia } \\
\text { Pascopyrum smithii - Stipa comata }\end{array}$ & \\
\hline Silty & $\begin{array}{l}\text { Elymus lanceolatus - Stipa comata } \\
\text { Pascopyrum smithii-Stipa comata } \\
\text { Pascopyrum smithii-Stipa comata- } \\
\text { Bouteloua gracilis } \\
\text { Pentaphylloides floribunda/Stipa curtiseta } \\
\end{array}$ & $\begin{array}{l}\text { Pascopyrum smithii-Stipa comata-Bouteloua } \\
\text { gracilis }\end{array}$ & $\begin{array}{l}\text { Elymus lanceolatus - (Koeleria macrantha) } \\
\text { Elymus lanceolatus - Stipa comata } \\
\text { Stipa curtiseta } \\
\text { Pascopyrum smithii-Stipa comata-Bouteloua } \\
\text { gracilis }\end{array}$ \\
\hline Clayey & Pascopyrum smithii-Bouteloua gracilis & Pascopyrum smithii & Elymus lanceolatus - (Koeleria macrantha) \\
\hline
\end{tabular}




\section{Appendix G . (cont.)}

\begin{tabular}{|l|l|l|c|}
\hline Range Site & Big Muddy Headwaters & Medicine Lake Area & Missouri Coteau Prairie Pothole \\
\hline
\end{tabular}

\begin{tabular}{|c|c|c|c|}
\hline Thin Hilly & $\begin{array}{l}\text { Schizachyrium scoparium - Muhlenbergia } \\
\text { cuspidata } \\
\text { Stipa comata - Muhlenbergia cuspidata }\end{array}$ & & \\
\hline $\begin{array}{c}\text { Shallow to } \\
\text { Gravel }\end{array}$ & & $\begin{array}{l}\text { Pascopyrum smithii-Stipa comata-Bouteloua } \\
\text { gracilis } \\
\text { Stipa comata - Bouteloua gracilis - Carex } \\
\text { filifolia }\end{array}$ & $\begin{array}{l}\text { Pascopyrum smithii-Stipa comata- } \\
\text { Bouteloua gracilis }\end{array}$ \\
\hline Dense Clay & $\begin{array}{l}\text { Sarcobatus vermiculatus/Pascopyrum } \\
\text { smithii }\end{array}$ & Pascopyrum smithii & \\
\hline
\end{tabular}

\begin{tabular}{|l|l||}
\hline Thin Breaks & $\begin{array}{l}\text { Juniperus horizontalis/Elymus lanceolatus } \\
\text { Eriogonum pauciflorum - Gutierrezia } \\
\text { Sarothrae } \\
\text { Pseudoroegneria spicatum }\end{array}$ \\
\hline
\end{tabular}

* Ecological units are represented by bold-faced blocks.

** "Herbaceous vegetation" is part of the plant association name for all plant associations in this table, with the exception of those having less than $25 \%$ cover of woody species, referred to as "shrub herbaceous vegetation" (including Artemisia cana and Sarcobatus vermiculatus types) and those having more than $25 \%$ cover of woody species, referred to as "shrubland (including Juniperus horizontalis and Pentaphylloides floribunda types). 


\section{Appendix I.}

\section{Sheridan County plant species of special concern and watch species}




\section{A gastache foeniculum (Pursh) Kuntze \\ Lavender hyssop \\ $M$ int Family (L amiaceae) \\ Global Rank: G4G5; State Rank: SU - watch.}

DESCRIPTION: Lavender hyssop is a rhizomatous perennial with erect, simple, or branched stems, $60-120 \mathrm{~cm}$ high (Figure G-1). The opposite leaves have broadly spade-shaped blades, $4-9 \mathrm{~cm}$ long, and petioles, 5-20 mm long. They are glabrous and green above but whitish with felt-like hairs below. The purplish flowers are borne in whorled clusters in a spike-like inflorescence, $4-8 \mathrm{~cm}$ long. The tubular corolla, 7-10 mm long, forms 2 lips at the mouth, and the tubular calyx is purple above with 5 triangular lobes. The 4 stamens are exserted from the mouth of the flower tube.

The other two wild hyssops in the state, Agastache urticifolia and A. cusickii, are western species with white corollas. Lavender hyssop vegetatively resembles catnip (Nepeta cataria), from which it differs in having a radially symmetrical calyx and lavendercolored flowers rather than a bilaterally symmetrical calyx and creamy white flowers with purple spots.

DISTRIBUTION: The center of its distribution is in the northern plains, extending from southern Ontario, west to Saskatchewan, and south to northern Colorado, northwestern Nebraska and northwestern Iowa (Great Plains Flora Association). While its distribution in Montana is mapped as spanning the eastern $1 / 4$ of the state (Great Plains Flora 1977), the records to support that distribution do not exist among herbaria in Montana and KANU. It has been reported in western Montana (Dorn 1984) but these are thought to represent places where it was cultivated in trial plantings. It was first collected in eastern Montana in 1988 by Lesica (\#4582; MONTU) from Richland County. It has also been documented in both Carter and Powder River County, on the Sioux and Ashland districts of the

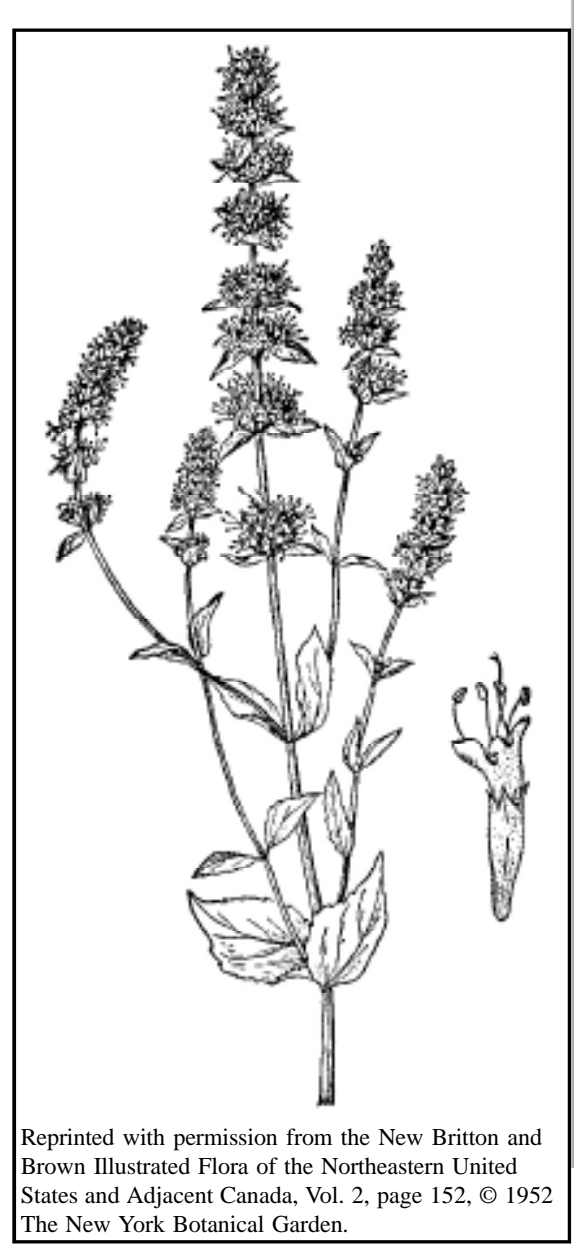

Figure G-1 Custer National Forest, respectively (Heidel and Marriott 1996). It was documented for the first time in Sheridan County southwest of Big Muddy Creek. This reflects the fourth county and the seventh site where it has been found, supporting the interpretation that it may be more widespread than previously known.

HABITAT: The rangewide habitat of lavender hyssop is characterized as "moist woodland, especially along streams or lakeshores, infrequent in open, wet ditches and prairies at higher elevations" (Great Plains Flora Association 1986). In northeastern Montana, it is restricted to wooded draws and escarpment slopes typically dominated by green ash (Fraxinus pensylvanica), in association with chokecherry (Prunus virginiana), Sprengel's sedge (Carex sprengelii), and northern bedstraw (Galium boreale). In southeastern Montana, it is restricted to relatively moist areas of tall shrub cover with many of the same associated species, but found within pine woodland.

COMMENTS: Lavender hyssop is thought to be more widespread than current records indicate in Montana, and the breadth of its distribution supports the case for dropping it from consideration if it is not in decline. However, its wooded draw habitat is limited and perhaps vulnerable. It also has cultural and commercial values, but is not known to be harvested from the wild in Montana, and is easily cultivated (Kindscher 1992). It remains on the watch list while its status is under review. 


\section{Agrimonia gryposepala Wallr.}

Common agrimony

Rose Family (R osaceae)

Global Rank: G5; State Rank: SU - watch.

Note: Assigned rank of SU and added to the watch list based in part on this study.

DESCRIPTION: Common agrimony is a perennial 30-150 $\mathrm{cm}$ tall with 1-several stems in small clumps arising from short, stout rhizomes and long fibrous roots (Figure G-2). Leaves are alternate, divided, coarsely-toothed, and glabrous above, with glands and hairs along the veins below. The yellow flowers are borne in a simple, elongated inflorescence with stalked flowers; they have 5 petals that are $3-5.5 \mathrm{~mm}$ long, and usually 15 stamens. The fruit is furrowed and shaped like a top or half-sphere, with rows of blunt, hooked bristles in rings on top; the outer ring usually reflexed. The achene is globose and $2.8-3.3 \mathrm{~mm}$ in diameter.

It is distinguished from Agrimonia striata by the absence of hairs in the furrows of the fruit, and the presence of glands along the axis of the inflorescence.

DISTRIBUTION: Common agrimony is widespread in eastern North American from Maine to Ontario and North Dakota, south to Kansas and North Carolina. It is reported for California and New Mexico (Great Plains Flora Association 1986). It was first collected in Montana at the north end of Bighorn Canyon National Recreation Area in 1983 and reported in Lichvar et al. (1985) for the Canyon. It was collected in northeastern Montana in the Big Muddy headwaters for the first time as part of this study.

HABITAT: Its rangewide habitat is characterized as "moist open woodlands, prairie ravines, and stream valleys" (Great Plains Flora Association 1986). It is a facultative wetland species (USDI Fish \& Wildlife Service 1994). In Sheridan County, it was found at only one wooded ravine along the breaks of the Upper Big Muddy Valley in shade of green ash (Fraxinus pennsylvanica) at the brushy margins of the ravine bottom with western snowberry (Symphoricarpos occidentalis) and Wood's rose (Rosa woodsii.)

COMMENTS: The status of common agrimony needs further review, hinging on the status of its habitat and vulnerability, as well as the number of records.

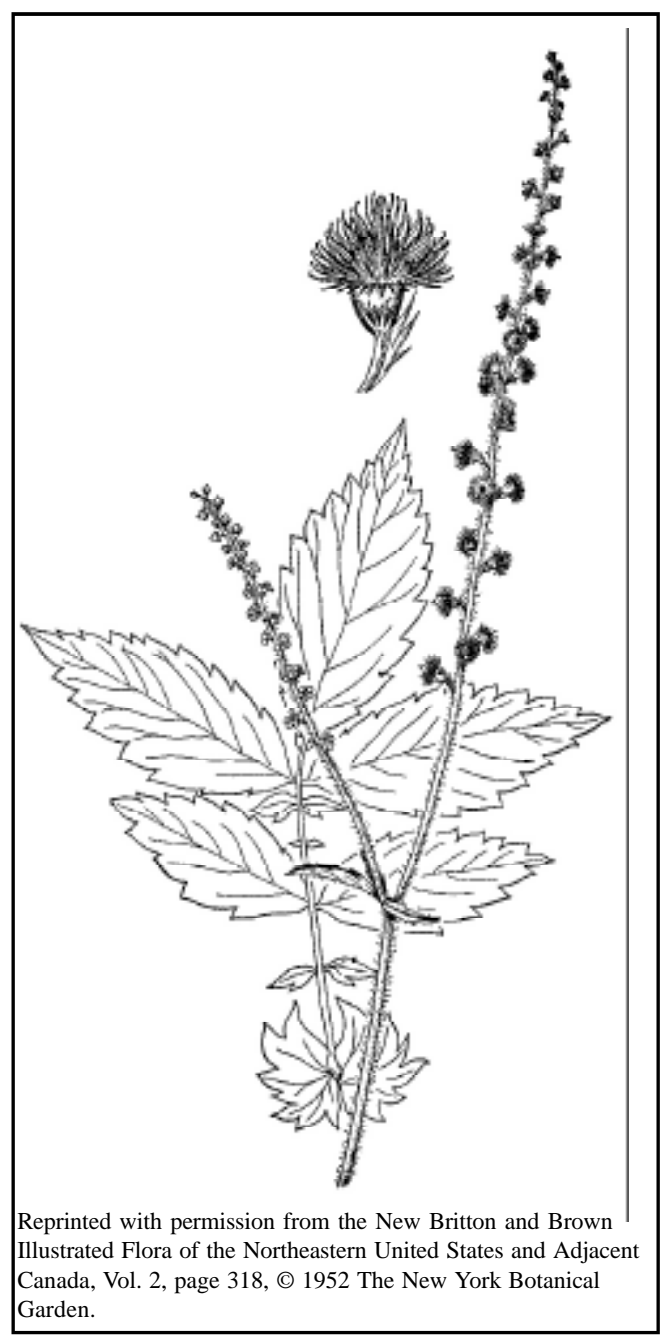

Figure G-2 


\title{
A sclepi as ovalifolia D cne.
}

\author{
$O$ valleaf milkweed \\ M ilkweed Family (Asclepiadaceae) \\ Global Rank: G5?; State Rank: S1
}

DESCRIPTION:: Ovalleaf milkweed is a perennial with usually solitary slender stems $20-60 \mathrm{~cm}$ high and arising from rhizomes (Figure G-3). The erect to spreading, opposite leaves have petioles that are up to $1 \mathrm{~cm}$ long, and broadly lance-shaped to elliptic blades that are $4-8 \mathrm{~cm}$ long with entire margins. Leaves are moderately long, hairy beneath, and the sap is milky. 1-3 umbrella-shaped, stalked clusters of 8-20 flowers are at the top of the stem or in the axils of the upper leaves. Each greenish-white to cream flower is $8-10 \mathrm{~mm}$ high and borne on a hairy stalk that is up to $20 \mathrm{~mm}$ long. Flowers have 5 reflexed corolla lobes that are 5-6 mm long, and 5 erect hoods that are 4-5 mm long and rounded at the tip; these hoods surround a greenish or yellowish central column, and each has a conical horn inside. The erect spindle-shaped fruits are 6-8 cm long and ca. $1 \mathrm{~cm}$ wide. Each fruit has numerous seeds, each with a light brown tuft of hairs that is $18-35 \mathrm{~mm}$ long. Flowering in late June-early July.

Green milkweed (Asclepias viridiflora) and showy milkweed ( $A$. speciosa) also have broad leaves, but the former lacks horns within the hoods, and the latter has pink to purple flowers.

DISTRIBUTION: The center of distribution is in the northern Great Plains, extending east as far as Wisconsin and Illinois; northwest to Alberta, and southwest to Wyoming. In Montana it was recently discovered for the first time in the southeastern corner of the state in Carter County (Heidel 1996). The Sheridan County occurrence is in the Missouri Coteau landscape north of Comertown. Its presence here represents the second known occurrence in the state and the first record from northeastern Montana.

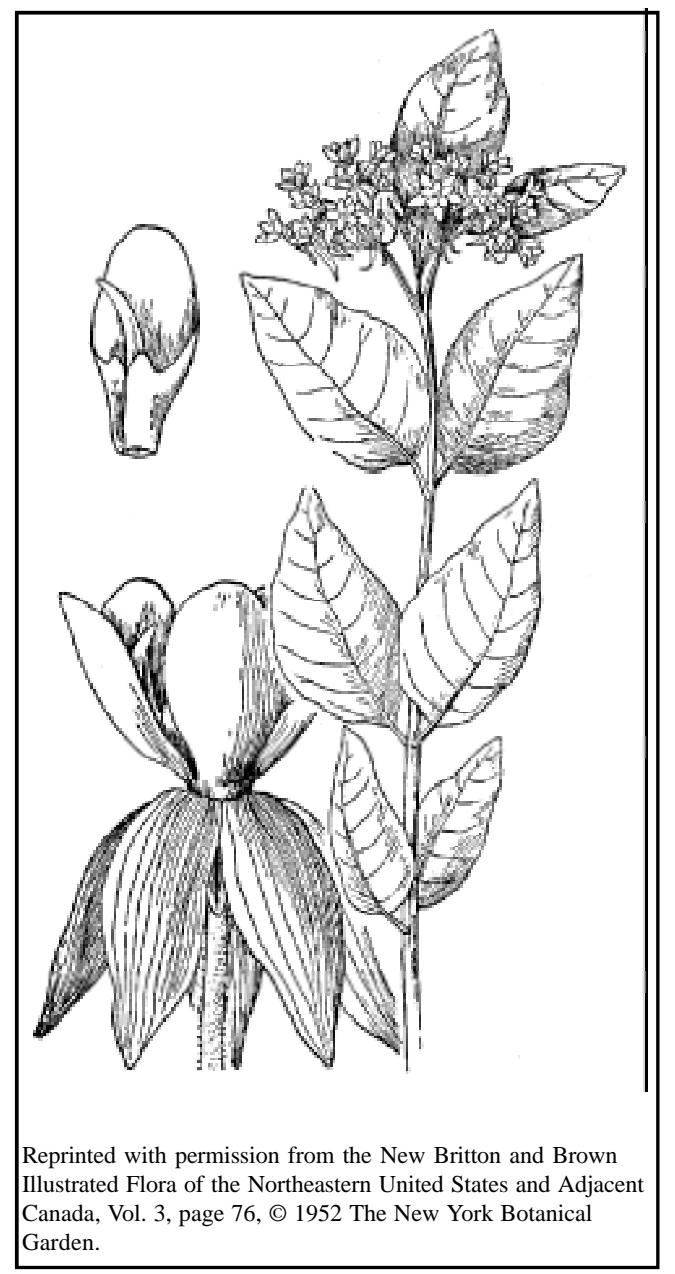

Figure G-3

HABITAT: Ovalleaf milkweed is restricted to mesic settings, including moist meadows in pineland escarpments of southeastern Montana, and rich grassland slopes on glacial till in northeastern Montana. The Sheridan County occurrence is on rolling prairie pothole terrain, at the crest of a northwest-facing slope dominated by northern porcupinegrass (Stipa curtiseta) below. Though this is a widespread habitat, no other locations of the species were found, and there is question whether this represents important habitat for the species.

COMMENTS: It persists under grazing, and is generally not preferred by stock, but the flowering stems and pods are eaten where it is in primary range. It is associated with Stipa curtiseta in Sheridan County, a grass with needles that deter grazing during flowering. The Carter County population was found in a wooded area that had burned as a summer groundfire about five years earlier.

It is sporadic at best in the prairie pothole landscape of Sheridan County but is present throughout the Missouri Coteau and Drift Plains counties of North Dakota (Great Plains Flora Committee 1977). Its presence in the floras of adjoining plains states and provinces suggests that it is under-documented in Montana, but its restriction to lush vegetation may also represent vulnerability. 


\section{C arex sychnocephala C arey \\ $M$ any-headed sedge \\ Sedge Family (Cyperaceae) \\ Global Rank: G4; State Rank: S1}

DESCRIPTION:: Many-headed sedge is a grass-like perennial that grows in clumps without rhizomes and has slender stems that are $5-50 \mathrm{~cm}$ high (Figure G-4). The lowest leaves are reduced to scales; the upper are flat and 1-4 mm wide. Flowers occur in 4-15 globose spikes clustered together at the top of the stem. The lower spikes are subtended by long, leaf-like bracts that greatly surpass the inflorescence. Inconspicuous male flowers are at the base of each spike. Pale green or tan perigynia are $5-7 \mathrm{~mm}$ long and ca. $1 \mathrm{~mm}$ wide and narrowly lance-shaped with a long beak and serrated margins. The pale, thin, lanceshaped scales have a green midvein and are ca. 1/2 the length of the perigynia that they subtend. Each perigynium has 2 stigmas and an achene that is lens-shaped. Fruit mature in July-August.

The combined characteristics of long, leaf-like lower bracts, and long, narrow perigynia distinguish this Carex from all other species in our area. It most closely resembles slender-beaked sedge (Carex athrostachya), which has much smaller bracts but similarities in appearance and habitat as found in the county.

DISTRIBUTION: Many-headed sedge is a northern species in North America, ranging from British Columbia to Ontario, and south to Washington, Iowa, and New York. In Montana, it is known from Sheridan and Glacier counties, a historic record in the Great Falls area, and the Flathead River and Tobacco River drainages. The Sheridan County occurrence was near Comertown in the Missouri Coteau area.

HABITAT: The species is restricted to moist soil of meadows along streams and ponds in the valleys and on the plains. It is a facultative wetland species (USF\&WS 1994). The 1987 collection of it in the Comerton area was described as wet meadow margin of prairie pothole, with foxtail barley (Hordeum jubatum) and water smartweed (Polygonum amphibium) in the wetland perimeter.

COMMENTS: Many-headed sedge is one of three species that was not be relocated in the course of this study, despite searches in the wetland margins where it had first been collected. It was originally collected from the County in 1987, a drought year, when it was noted as rare. It is not known whether this species remains vegetative when there is taller dense vegetation. As part of the rest-rotation grazing regime, it would be most insightful to survey for it in midsummer during the year it was subject to early-season grazing. It is among a group of species benefiting by restoration of wetland margins. 


\section{Centunculusminimus(L.) Krause Chaffweed \\ Primrose Family (Primulaceae) \\ Global Rank: G5; State Rank: S2}

Note: Reranked from S1 to S2 based in part on results of this study.

DESCRIPTION:: Chaffweed is an annual with prostrate or erect stems, $2-10 \mathrm{~cm}$ long, that are branched near the base and root at the nodes (Figure G-5; SID 849). The alternate leaves, 5-10 $\mathrm{mm}$ long, are egg to spoon-shaped with entire margins. Foliage is glabrous. Solitary, inconspicuous flowers on short stalks occur in the leaf axils. Each flower has a deeply 4-lobed calyx, 2-3 mm long, and a small, pink, 4-lobed, tubular corolla, ca. $1 \mathrm{~mm}$ long, that withers on the maturing ovary. There are 4-5 stamens, and the fruit is a globose capsule that is ca. $2 \mathrm{~mm}$ long. Flowering and fruiting June-September.

Sea milk-wort (Glaux maritima) is similar but has lower leaves that are opposite each other and it is perennial. A hand lens or microscope may be needed for positive identification.

DISTRIBUTION: Chaffweed is native to Europe and North America, widely but sparsely distributed from. Nova Scotia to Saskatchewan, south to Florida and Mexico.

In Montana, it is known from the Bitterroot Valley; from a historical record in the Flathead Valley, a historical record in

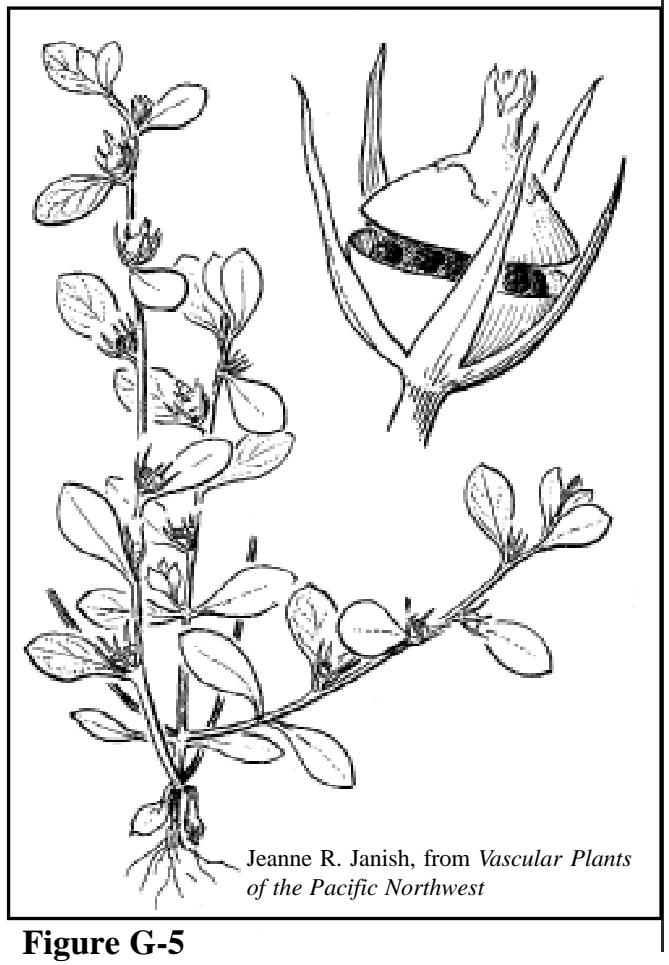
Missouri River wetlands near Great Falls area, and from Sheridan and Valley counties for a total of 7 extant records and 3 historical ones. The Sheridan County occurrences are in the Missouri Coteau area north of Comertown.

HABITAT: Chaffweed grows in vernally wet, sparsely vegetated soil around ponds and along rivers and streams in the valleys and on the plains. It is an obligate wetland species (USF\&WS 1994).

It occupies the margins of seasonal wetlands in Sheridan County, on silty soils that dry out early in the season. The vegetation is relatively low-growing, including the following associated species: needle spike-rush (Eleocharis acicularis), western wheatgrass (Pascopyrum smithii), mat muhly (Muhlenbergia richardsonis), ticklegrass (Agrostis scabra), and annual forbs like purslane speedwell (Veronica peregrina) and field chickweed (Cerastium arvense).

COMMENTS: Its habitat is not affected by trampling except when soils are saturated in spring. It is among a group of species potentially benefiting by restoration of wetland margins. Kentucky bluegrass (Poa pratensis) is sometimes present and assumes dominance, competing with chaffweed. 


\section{Chenopodi um subglabrum (S. Wats.) A. N els. \\ Smooth goosefoot \\ Goosefoot Family (Chenopodiaceae) \\ Global Rank: G 4; State Rank: S1}

Note: Global rank was changed from G3 to G4 based on consultation with botanists in Nebraska and Wyoming.

DESCRIPTION: Smooth goosefoot is an annual with erect, simple or more often branched stems usually $10-40 \mathrm{~cm}$ high (Figure G-6; SID 1104). The alternate leaves are linear with entire margins, single veined, glabrous, up to $3 \mathrm{~cm}$ long. Flowers are small, green, and grouped in remote clusters on the branched stem. Each flower lacks petals but has 5 glabrous sepals and 5 stamens. The 1-seeded fruit is compressed, hemispherical and relatively large; $1.2-1.6 \mathrm{~mm}$ across, exposing a jet-black fruit at maturity that readily separates from the pericarp. Fruiting in late June-July.

Chenopodium subglabrum is related to slimleaf goosefoot $(C$. leptophyllum) and desert goosefoot (C. dessicatum) but it does not have a farinose perianth surface. It tends to have larger fruits 1.2$1.6 \mathrm{~mm}$ in diameter and more widely spaced glomerules. It is further distinguished from the latter in that it has leaves singleveined rather than 3-veined from the base.

DISTRIBUTION: Smooth goosefoot is centered in Nebraska and South Dakota, also documented from Manitoba to Alberta south to Kansas, Utah and Nevada (Crawford 1973, and Biosource 1999). Plants of the Pacific Northwest are said to differ from Great Plains material (Crawford 1973) and their proper disposition has not been resolved. It is also reported to occur sparsely in the Midwest, treated in early floras as a synonym of C. leptophyllum; further specimen review may be needed.

In Montana, it is known from 5 occurrences in Carter, Cascade and Sheridan counties, and one record in Custer County that may be extirpated. The Sheridan County occurrence is in the Medicine Lake sandhills.

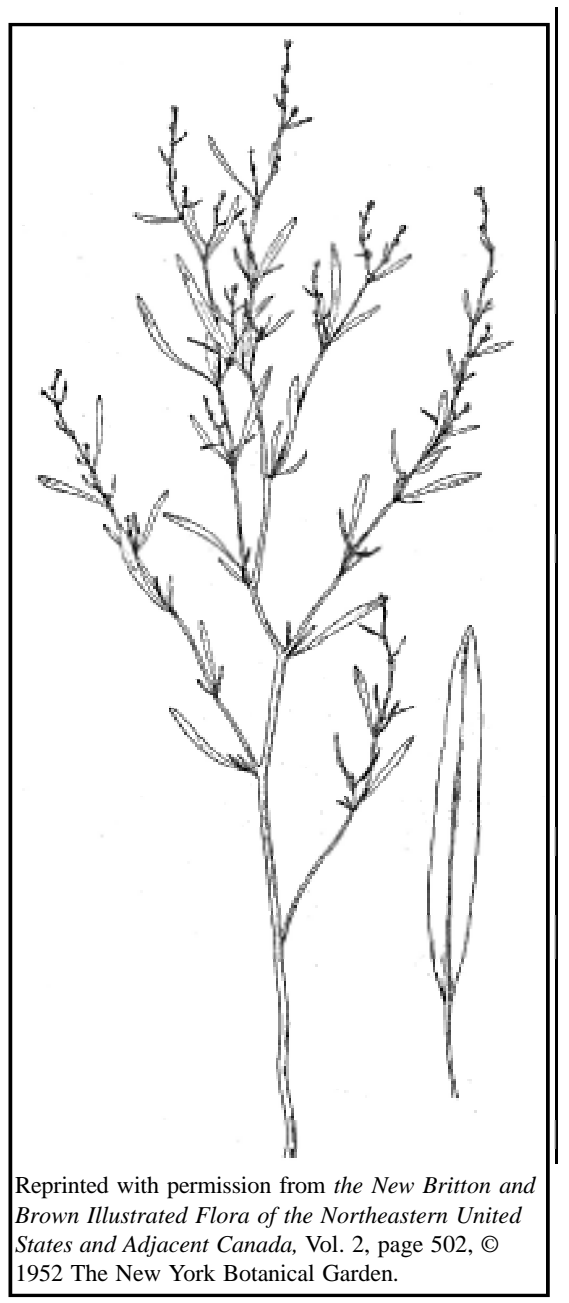

Figure G-6

HABITAT: It occupies sparsely vegetated sand dunes and sandy terraces of major rivers on the plains; at early stages of succession in both settings. In the study area it is restricted to active blowouts or lee slope deposits; on the loose sand at the side of the "bowl" or on the rim. Associated species include indian ricegrass (Oryzopsis hymenoides), lemon scurfpea (Psoralidium lanceolatum), and Fendler cat's-eye (Cryptantha fendleri).

COMMENTS: Maintenance of early seral habitat in the upland settings may require some level of disturbance, i.e., periodic reduction in grass cover. Maintenance of early seral habitat in the riparian setting may require intact flow regimes and landscape processes over a larger scale. Tamarisk control is needed in riparian habitat of southeastern Montana. 


\section{Cryptantha fendleri ( $G$ ray) G reene \\ Fendler cat's-eye \\ Borage Family (Boraginaceae) \\ Global Rank: G4; State Rank: S2}

Note: Reranked from $\mathrm{S} 1$ to $\mathrm{S} 2$ based on results of this study.

DESCRIPTION: Fendler cat's-eye is an annual with simple or branched stems that are $5-20 \mathrm{~cm}$ high (Figure G-7). The alternate, narrow, strap-shaped leaves may be up to $\mathrm{cm}$ long; those at the base are usually brown by the time the plant is fruiting. Foliage is sparsely covered with spreading hairs. Tiny white flowers are borne on coiled stalks that unwind and elongate as flowering progresses from the base upward. The corolla is ca. $1 \mathrm{~mm}$ high and has a small, united portion below and 5 spreading petals above. The calyx is covered with stiff, straight hairs and becomes 4-6mm long in fruit. Within each fruiting calyx are 4 smooth, shiny, narrowly lance-shaped nutlets that are ca. $1.5 \mathrm{~mm}$ long and $1 / 3$ as wide; 1 or more of these may be missing. Flowering in May-early July.

Annual species of Cryptantha are distinguished by characters of the seeds. Fendler cat's-eye (Cryptantha fendleri) is distinguished by having 4 smooth, shiny nutlets that are lance-shaped and $1 / 3$ as wide as they are long. A hand lens or microscope is needed for positive identification.

DISTRIBUTION: Fendler cat's-eye is a western North American species distributed from southern British Columbia to Saskacthewan, and south to Oregon, New Mexico, and Nebraska. In Montana, the species is know from two large sandhill areas at opposite corners of the state; the Centennial sandhills in Beaverhead County, and the Medicine Lake sandhills in Sheridan County. In addition, there was a 1943 record from north of the Medicine Lake sandhills in a roadside setting (Hotchkiss \#6900 MONT). It may indicate that the species was once more widespread across gentle sandplains, that it was more widespread around the time of drought years, and/or that it may be adventive in roadside settings under some conditions.

HABITAT: Open areas of sand dunes in the valleys and on the plains provide habitat for Fendler cat's-eye. It occupies a range of sparsely-vegetated settings and successional stages, as has been documented in the Centennial Valley (Lesica and Cooper 1999). It is the most widespread of the species sought in the Medicine Lake Sandhills. It is not only found in blowout, but throughout much of the gentler sandplains where grass cover is discontinuous.

COMMENT: Fendler's cat's-eye does not grow among tall, dense grasses. The widespread practice of seeding sandhills to crested wheatgrass (Agrpopyron cristatum) has reduced available habitat. It was found to persist under ongoing heavy grazing, and persists where brittle pricklypear (Opuntia fragilis) is common. The abundance of cactus may indicate past heavy grazing. A conceptual management framework involving this and other sandhills species is presented in Lesica and Cooper (1999) for southwestern Montana. Invasion of leafy spurge (Euphorbia esula) is also a potential threat.

The series of scattered plants in the Medicine Lake sandhills are taken to represent one giant population complex; the largest of two in the state. Its state rank was changed because it is unaffected by most management actions, but it was retained as a species of special concern because it is still considered vulnerable. 


\section{C yper us schwei nitzi i T orr. \\ Schweinitz' flatsedge \\ Sedge Family (C yperaceae) \\ Global Rank: G5; State Rank: S2}

DESCRIPTION: Schweinitz' flatsedge is a grass-like perennial with stems that are $10-40 \mathrm{~cm}$ high, arising from short, irregularly swollen rhizomes (Figure G-8). The leaves are 1-4 $\mathrm{mm}$ wide and located mostly near the base of the stem. The inflorescence is subtended by 3-6 long, leaf-like bracts, some of which are wider than the leaves. The infloresence is made up of ascending clusters of flattened spikelets that are 5-25 $\mathrm{mm}$ long and borne on stalks that are very short to long. The flowers are crowded opposite each other and consist only of a small, pointed scale, that is ca. 3-4 mm long and subtends 3 stamens and an ovary. The seed is triangular in cross-section. Fruit mature in late June-July.

This is our only perennial Cyperus and is the only one occurring in upland habitat.

DISTRIBUTION: Schweinitz' flatsedge extends from Alberta to New Mexico and east from Quebec to West Virginia. It is concentrated in the Great Lakes and the Great Plains. In Montana, it is known from eight records in five eastern counties. The Sheridan County occurrence is in the Medicine Lake sandhills area.

HABITAT: Schweinitz' flatsedge occupies sparsely vegetated sand dunes, in blowouts and open ridges. It is often on loose sand slopes in the "bowl" of a blowout. It is associated with indian ricegrass (Oryzopsis hymenoides), needle-and-thread (Stipa comata), lemon scurpea (Psoralidium lanceolatum), and Fendler cat's-eye (Cryptantha fendleri.)

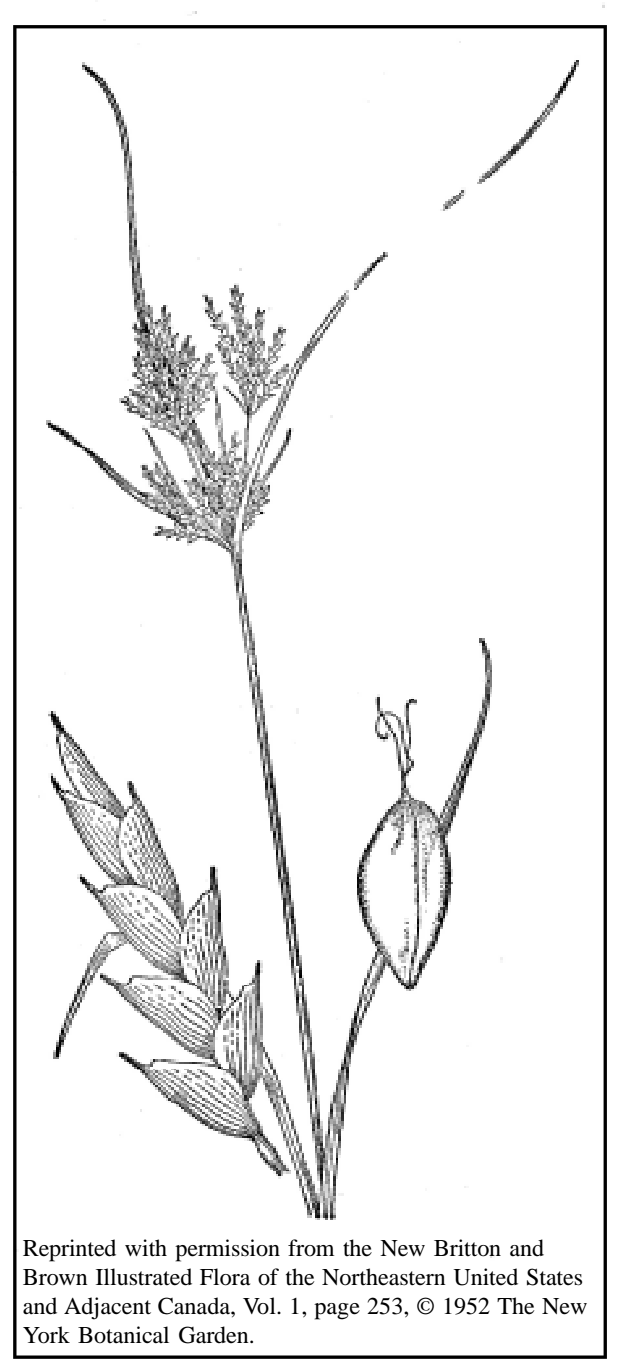

Figure G-8

COMMENTS: Maintenance of early seral sandhill habitat may require some level of disturbance, i.e., periodic reduction in grass cover. It is present in low numbers on the rolling sandplains where brittle pricklypear (Opuntia fragilis) is abundant and may reflect past heavy grazing.

The series of scattered plants in the Medicine Lake sandhills are estimated at 1,000+ plants and are taken to represent one giant population complex; the largest in the state. 


\section{D alea villosa (N utt.) Spreng. var. villosa \\ Silky prairie clover \\ Bean Family (Fabaceae or L eguminasae) \\ Global Rank:G5T?; State Rank: S1}

DESCRIPTION: Silky prairie clover is a perennial with 1-many ascending or lax, branched stems that are $20-40 \mathrm{~cm}$ high, and arising from red-orange roots and a rootcrown (Figure G-9). The alternate, pinnately compound leaves are $2-4 \mathrm{~cm}$ long and have 1121 linear leaflets. The foliage has numerous sunken glands with dense long hairs. The pink to rose-purple flowers are densely crowded in cylindrical spikes that are $3-12 \mathrm{~cm}$ long, at the ends of stems and branches. Each flower is 4-6 mm long and has 4 separate petals, a densely spreading, hairy, 5-lobed, cup-shaped calyx, and 5 stamens that are usually longer than the petals. The narrowly egg-shaped pods are 2-3 mm long and densely long and hairy.

Other Dalea in Montana usually have 11 or more leaflets, and are not as conspicuously hairy. The combination of the long, hairy calyx and the 5 stamens further separate this species from other Dalea and from species of Psoralidium and Amorpha.

DISTRIBUTION: This species has its center of distribution in the Great Plains, ranging from Saskatchewan and Manitoba to Wisconsin, and south to Colorado and Texas. In Montana, it is known from four records in Carter, Richland and Sheridan counties. The Sheridan County occurrence is in the Medicine Lake area on gentle sandplains outside of the sandhills. It is likely to occur in the sandhills but was not found in survey to date.

HABITAT: Silky prairie clover grows on loose sand of sand dunes or wind-eroded deposits around sandstone outcrops. Its habitat in Sheridan County is incompletely documented or different in that it was not found growing on any of the loose sand in sand dunes, but only at erosion points along a section-line road and a nearby pasture 2-track. In this setting, it grows literally at the edge of loose sand, in greatest numbers where prairie sandreed

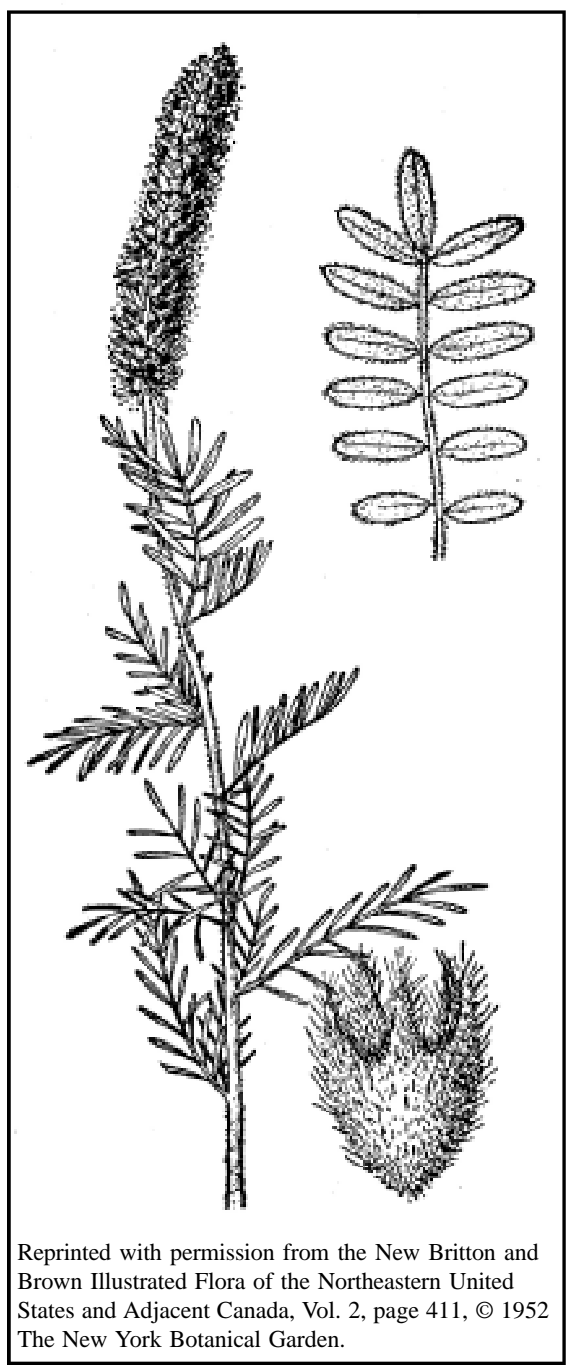

Figure G -9 (Calamovilfa longifolia) and sandhill bluestem (Andropogon hallii) are abundant, but also present with needle-and-thread (Stipa comata). The "natural" habitat for silky prairieclover elsewhere in the state is in or at the edge of small blowouts, representing early successional habitat and the Oryzopsis hymenoides - Psoralidium lanceolatum plant association.

COMMENTS: This species is favored by some level of disturbance and may decline in the absence of disturbance. It is also palatable, and a decreaser under grazing (Smith 1976), with limited capacity to resprout. 


\section{Lobelia kalmi L. \\ Kalm's lobelia \\ Bellflower Family (C ampanulaceae) \\ Global Rank: G5; State Rank: SU}

Note: Assigned rank of SU and added to watch list in part based on results of this study.

DESCRIPTION: Kalm's lobelia is a slender perennial $10-50 \mathrm{~cm}$ tall, with a single branched stem arising from a small basal rosette of leaves and a slender rhizome (Figure G-10). Basal leaves are narrower at the base than the tip, with rounded tips. Stem leaves are sessile, 0.5-3 $\mathrm{mm}$ wide with pointed tips, and decrease in size toward the top of the stem. The elongated infloresence is made up of a loose series of 2-12 flowers on short stalks. The corolla is pale to dark blue, 7-10 mm long, with lower lobes curving downward.

Distinguished from Lobelia spicata by the stem leaves $3 \mathrm{~m}$ or less wide, and usually a much narrower stem 1.5 or less in diameter.

DISTRIBUTION: Kalm's lobelia is a boreal species known from Nova Scotia to Alberta, south to Pennsylvania, Iowa, North Dakota, Colorado and northeastern Washington. In Montana, it is known 5 historic collections in Flathead County, 1 historic collection in Missoula Co., and a historic collection that described the location simply as "Missouri Valley".

In Sheridan County, it was first documented by a 1943 collection record near Dagmar that corresponds with the population in this study, where it occurs in one widely-scattered population complex across four sections along Lake Creek and associated potholes.

HABITAT: In Montana, it is restricted to rich fens, and is an obligate wetland species (USF\&WS 1994). In Sheridan County, it is in small areas of patterned peatland, with distinctive parallel mound-pool (string-flark) bands (Chadde et al. 1998). The dominant species in its untrampled habitat is mainly few-flowered spikerush (Eleocharis quinqueflora), and associated species include green sedge (Carex viridula), rush aster (Aster junciformis), mat muhly (Muhlenbergia richardsonis), jointed rush (Juncus articulatus), marsh arrowgrass (Triglochin palustre), wedgegrass (Sphenopholis obtusata), northern bog violet (Viola nephrophylla), and small-flowered grass-of-parnassus (Parnassia palustris).

COMMENTS: This species persists with grazing, but is impacted by heavy prolonged trampling that changes the mound-pool microtopography and increases competing vegetation cover. The associated species that increase under trampling include common arrowgrass (Triglochin maritimum), common threesquare (Scirpus pungens), and water sedge (Carex aquatilis). Species records have not been compiled from all Montana herbaria to date and it may be considered for addition to the species of special concern list pending further status review. 


\section{Lobelia spicata Lam. \\ Pale-spiked lobelia \\ Bellflower Family (C ampanulaceae) \\ Global Rank: G5; State Rank: S1}

Note: Reranked from SH to S1 based on results of this study.

DESCRIPTION: Pale-spiked lobelia is a perennial with unbranched stems that are 20-80 cm high (Figure G-11). The oblong, lance-shaped stem leaves are 5-10 $\mathrm{cm}$ long and $0.4-2 \mathrm{~cm}$ wide, have toothed margins and short petioles, and become smaller up the stem. Foliage is glabrous to sparsely short-hairy. Flowers are 7-12 mm long and are born on short, 2-5 mm long stalks in a sparingly branched, narrow, crowded inflorescence. Each flower has 5 narrow sepals that are 5-9 $\mathrm{mm}$ long and a light blue, tubular, $4-10 \mathrm{~mm}$ long corolla that flares into a spreading 2-lobed upper lip and a 3-lobed lower lip. The ovary is below the base of the corolla and matures into a nearly globose capsule that is $3-5 \mathrm{~mm}$ high.

Kalm's lobelia (Lobelia kalmii) has linear stem leaves $0.3 \mathrm{~cm}$ or less wide, and is confined to saturated soils. Most other species of Campanulaceae with similar flowers are annuals.

DISTRIBUTION: Pale-spiked lobelia occurs in grassland landscapes from Ontario west to Saskatchewan, and south from Georgia to Texas. In Montana, it is known from two historic records including a 1924 collection in Richland County and a 1943 collection in Sheridan County near Medicine Lake. The historic record near Medicine Lake ("4 miles northeast of Medicine Lake") is thought to give directions from the Lake rather than the Town. This would put it in the Lake Creek Valley, but it was not relocated here. In this study, two more occurrences were documented in sandplains below Brush Mountain and in the Missouri Coteau prairie potholes.

HABITAT: Pale-spike lobelia grows in a variety of moist meadow settings on the plains. It is a facultative wetland species (USF\&WS 1994).

In Sheridan County, it was found mainly in narrow bands along wetland margins, including prairie potholes and shallow meandered wetlands in outwash channel. The historic Medicine Lake collection was made from a setting described as "tallgrass." The associated vegetation of the two more recent records is dominated by clustered field sedge (Carex praegracilis), mat muhly

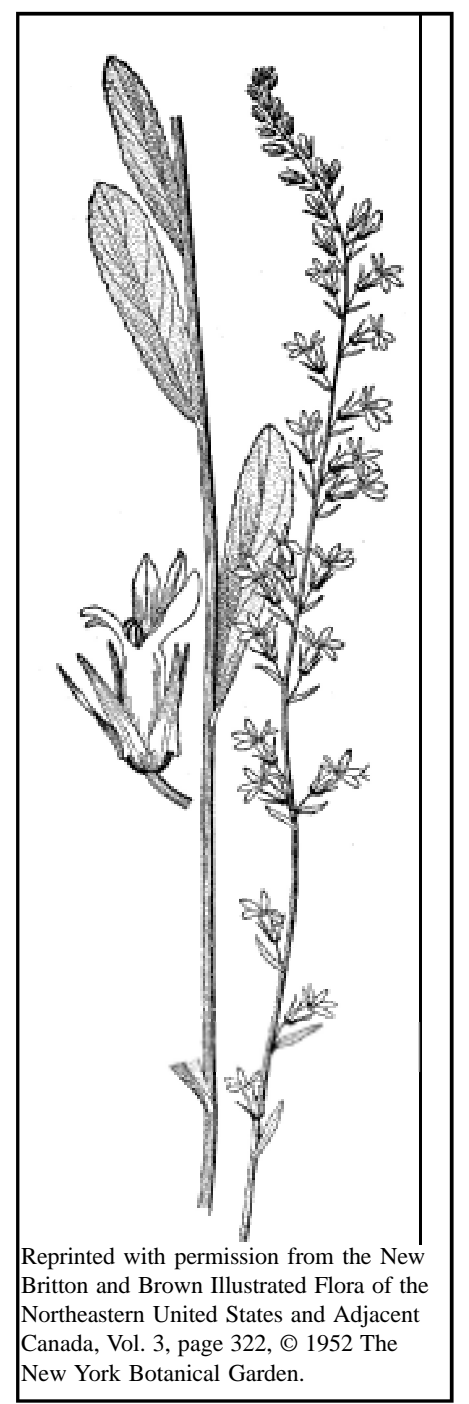

Figure G-11 (Muhlenbergia richardsonis), western wheatgrass (Pascopyrum smithii) or switchgrass (Panicum virgatum); and other species present include meadow anemone (Anemone canadensis), mountain blue-eyed-grass (Sisyrinchium montanum), northern reedgrass (Calamagrostis stricta), wedgegrass (Sphenopholis obtusata), and wild licorice (Glycyrrhiza lepidota).

COMMENTS: Exotic species like Kentucky bluegrass (Poa pratensis) and ticklegrass (Agrostis scabra) are invasive and competitive in this species' habitat. Its response to grazing is not known but the pastures where it occurs are not grazed during flowering time. It is among a group of species potentially benefiting by restoration of wetland margins. The two populations in this study represent the only two records of recent decades in the state. 


\section{Phlox andicola E. N els. Plains phlox \\ Phlox Family (Polemoniaceae) \\ Global Rank: G4; State Rank: S2}

DESCRIPTION: Plains phlox is a perennial with loosely tufted stems that are $4-10 \mathrm{~cm}$ high arising from creeping rhizomes (Figure G-12). The 5-8 pairs of opposite, linear leaves have prominent midveins and whitish bases and are 10-25 mm long, ca. $1 \mathrm{~mm}$ wide and come to a sharp point. Foliage is glabrous to sparsely hairy. Stems are white. 1-5 white flowers are borne at the stem tips. Each flower has 5 petals and a tubular corolla. The calyx is also tubular, with 5 lobes, tangled long hairs, and 6-11 mm length. Flowering in May-early June.

Distinguished from Hood's phlox (Phlox hoodii) by leaf length over $10 \mathrm{~mm}$ long, and from alyssum-leaved phlox ( $P$. alyssifolia) by leaf width less than $2 \mathrm{~mm}$ wide. Flowers are needed for positive identification, and hybridization between these species is reported elsewhere in the range.

DISTRIBUTION: Plains phlox is a Great

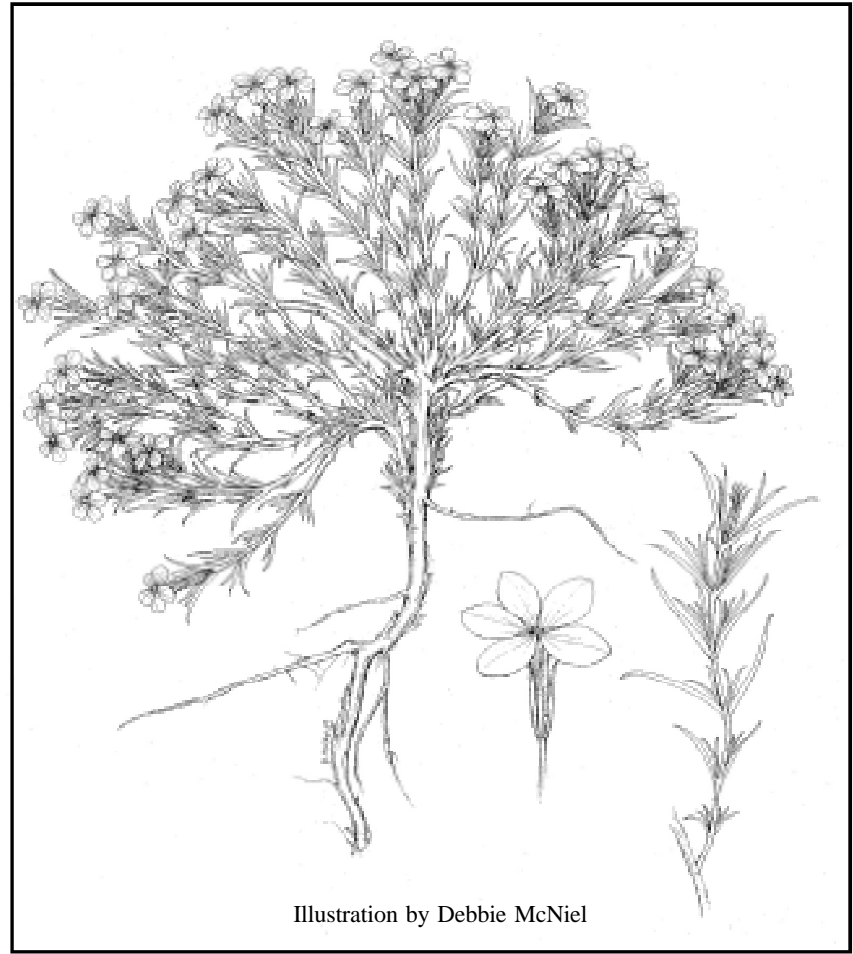

Figure G-12 Plains species ranging from Montana and North Dakota, south to Colorado and Kansas. In Montana, it is known from ten occurrences in five eastern counties. All of the known Sheridan County occurrences are on sandplains north and east of the Medicine Lake sandhills.

HABITAT: It occupies sandy soil of grasslands and open pine woodlands on the plains as a mid-successional species. Unlike most of the other sand-loving species surveyed in this study, it does not occupy dunes, and often grows in relatively dense vegetation cover. It is found on a variety of sandplains and in loose sand below sandstone outcrops. In Sheridan County,

COMMENTS: Plains phlox is an early-blooming plant that was not surveyed before late June in this study. Further survey is warranted. It occupies well-established grassland, but may benefit by periodic reduction in canopy cover. One of the places where it was found in highest numbers was a calving pasture.

The Sheridan County records represent the largest known numbers of records and total plants in the state. 


\section{Primula incana M.E. Jones \\ M ealy primrose \\ Primrose Family (Primulaceae) \\ Global Rank: G4G5; State Rank: S2}

DESCRIPTION: Mealy primrose is a perennial with leafless stems $10-45 \mathrm{~cm}$ tall, arising from a basal rosette of leaves and fibrous roots (Figure G-13). Leaves usually have a mealy, whitish covering, are elliptic or oblanceolate with slightly toothed margins, $0.3-1.6 \mathrm{~cm}$ wide, and up to $6 \mathrm{~cm}$ long Including the winged petioles. Infloresences are capitate, 7-19 flowered, surmounting involucral bracts that are $0.5-1 \mathrm{~cm}$ long, oblong, with a whitish, mealy covering, and pouched at the base. The calyx also has a whitish, mealy covering, is cylindrical, $0.4-0.7 \mathrm{~cm}$ long, and divided up to $1 / 3$ its length by lanceolate teeth that have a glandular surface. The corolla is lavender with a yellow throat, forming a tube that is equal or slightly longer than the calyx, app. $0.4-0.8 \mathrm{~cm}$ long and wide. Stamens are ca. $1 \mathrm{~mm}$ long and of equal length. The capsule is cylindrical to slightly elliptical, and equaling or slightly exceeding the length of the calyx. Flowering in June.

Primula incana differs from Parry's primrose ( $P$. parrryi) in that the corolla is less than $1 \mathrm{~cm}$ wide, the leaves lie low to the ground, and it is not found at alpine or subalpine habitats. It most closely resembles Idaho primrose ( $P$. alcalina), not known from eastern Montana, which it also differs in having a lilac rather than white corolla, and generally longer calyx and corolla.

DISTRIBUTION: Mealy primrose is a boreal species ranging from Alaska to Ontario, extending south to Utah, Colorado, and North Dakota. It was only known from western Montana prior to this study, documented from the Upper Clarks Fork River, Jefferson River, and Madison River drainages, Front Range, and historically known from the Beartooth Mountains. In Sheridan County, it is

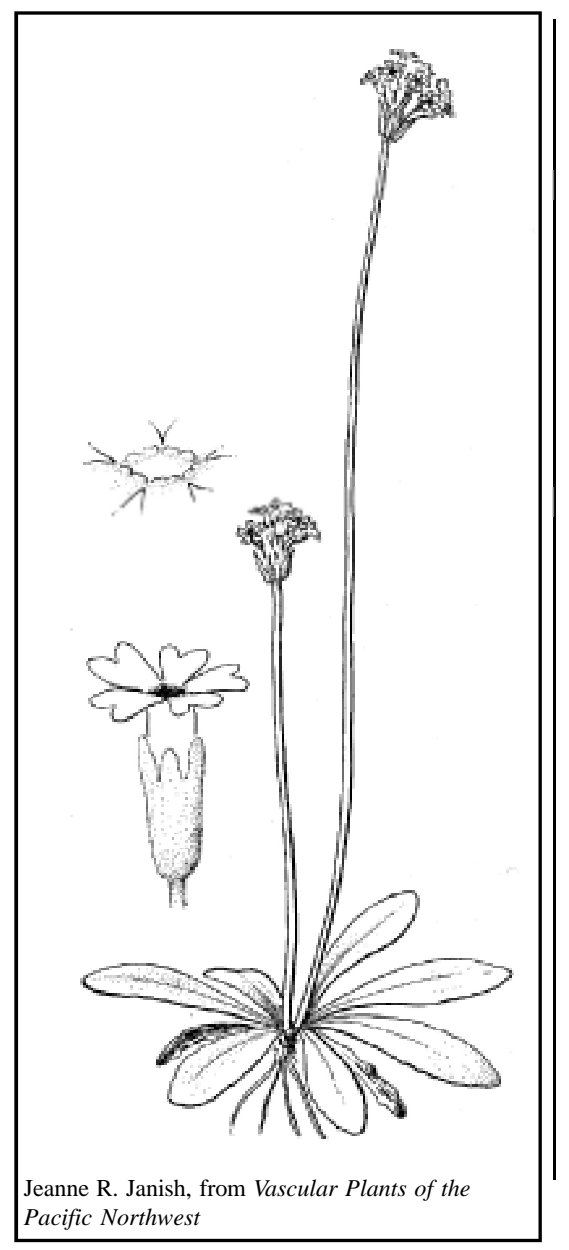

Figure G-13 known only from one occurrence in the Missouri Coteau area. Of the 20 records in the state, several are historic.

HABITAT: This species occupies alkaline meadows or peatlands on the plains, and in valley and foothill zones. It is a facultative wetland species (USF\&WS 1994). In Sheridan County it was restricted to seep habitat with marl deposits beside an alkali lake. The most common associated species are Baltic rush (Juncus balticus), common threesquare (Scirpus pungens), common arrowgrass (Triglochin maritimum), and common silverweed (Potentilla anserina). Other species present include mat muhly (Muhlenbergia richardsonis), golden sedge (Carex aurea), Parry's sedge (C. parryana), wooly sedge ( $C$. lanuginosa), small-flowered grassof-parnassus (Parnassia palustris), mountain blue-eyed-grass (Sisyrinchium montanum) and sea-milkwort (Glaux maritima). This species occupies the same habitat as Kalm's Lobelia (Lobelia kalmii) on Great Plains peatlands outside of Montana, but they were not found together in Sheridan County.

COMMENTS: Like Lobelia kalmii, it may be eliminated by heavy prolonged trampling that changes the mound-pool microtopography and increases competing vegetation cover. The Sheridan County occurrence is the only known occurrence in eastern Montana. 


\section{Sci rpus heter ochaetus C hase \\ Slender bulrush \\ Sedge Family (C yperaceae) \\ Global Rank: G5; State Rank: S1}

DESCRIPTION: Slender bulrush is a tall, glabrous, grass-like perennial with firm, round stems that are 1-2 $\mathrm{m}$ tall and arising from stout brown rhizomes (Figure G-14). The few leaves are borne near the base and have a sheath surrounding the stem, but the blade is small and tapers to a point. Numerous slender-stalked, solitary spikelets are borne on the spreading branch tips of an open, terminal inflorescence. Each egg-shaped spikelet is up to $17 \mathrm{~mm}$ long and has several flowers arranged in a tight spiral. Flowers consist of a single scale that is up to $5 \mathrm{~mm}$ long; it subtends 3 stamens and an ovary with 4 white bristles that are as long as the scales and arising from the base. The 3 -sided achene is ca. 3 $\mathrm{mm}$ long with a short beak and 3 stigmas. Fruiting in August.

The common hardstem bulrush (Scirpus acutus) and softstem bulrush ( $S$. validus) are very similar but have 2 stigmas and sessile spikelets arranged in small clusters.

DISTRIBUTION: Slender bulrush is distributed

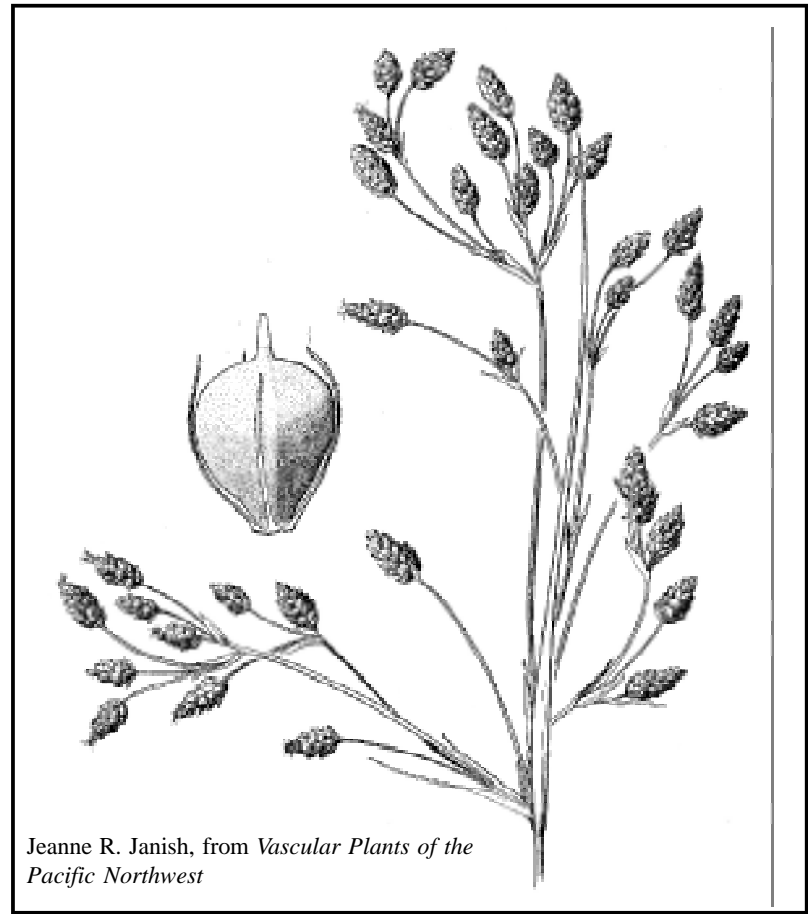

Figure G-14 from Washington and Oregon east to Quebec and Massachusetts, centered in the Great Plains and extending south to Oklahoma and Kentucky.

In Montana, it is known from only three occurrences to date, including wetlands near the Flathead Valley and in northeastern Montana in the Medicine Lake area.

HABITAT: Slender bulrush occupies semi-permanent marshes and edges of lakes and ponds on the plains. It is an obligate wetland species (USF\&WS 1994). It is common or abundant under "fresh" water conditions elsewhere in the Missouri Coteau ( $>225 \mu \mathrm{mhos} / \mathrm{cm}$; Stewart and Kantrud 1972). It was reported as "locally common" in association with Eleocharis palustris at its only other collection site in Montana from Phillips County. Its habitat at the site of the 1943 collection in Sheridan County was described only as "marsh."

COMMENTS: Slender bulrush is one of three species that was not relocated in the course of this study. It was described as "abundant" where it was originally collected on the Refuge in 1943. Wetland habitat in parts of the Lake Creek Valley was subsequently altered by a system of levees to increase open water habitat; implications for the species are unknown. The limited surveys on the Refuge to date should be expanded and any available water chemistry data used to identify and search wetlands with "fresh" water condition. 


\section{Si syri inchi um sptentri onale Bickn. \\ $\mathrm{N}$ orthern blue-eyed grass \\ I ris Family (I ridaceae) \\ Global Rank: G3G 4; State Rank: S1}

DESCRIPTION: Northern blue-eyed grass is a tuft-forming perennial, app. 20-30 cm tall, arising from fibrous roots (Figure G-15). Light green leaves are up to $2 / 3$ the height of the stem and 1-2.3 mm wide. Stems are $1-1.9 \mathrm{~mm}$ wide, surmounted by terminal infloresences. The outer bract is 24-55 mm long, nearly twice the length of the inner bract or longer, united basally $1.2-2.8 \mathrm{~mm}$. The inner bract has broad, clear margins and is not swollen at the base. 1-5 flowers are borne on slender stalks app. same length as the inner bract. Tepals are blue-violet with yellow bases; the outer are $9-12 \mathrm{~mm}$ long. The capsule is $2.7-5 \mathrm{~mm}$ long. Flowering in June.

It is distinguished from the mountain blue-eyed grass (Sisyrinchium montanum) in not having an inner bract with a swollen base, or an outer bract that widens above the tip of the inner bract. Stems are usually narrower; $1.9 \mathrm{~mm}$ or less wide.

Note: This species is not treated consistently between floras, and the information above represents the revisionary taxonomic work presented in Cholewa and Henderson (1984) for the genus in western states. It is otherwise treated as part of Sisyrinchium angustifolium in the flora of the Great Plains (Great Plains Flora Association 1986) and of the Canadian provinces (Scoggan 1978). Many authors treat S. angustifolium as synonymous with $S$. montanum (e.g. Dorn 1984). Of these three closelyrelated species, only two are in Montana, and further taxonomic review is underway as part of the Flora of North America project to clarify the distribution and status of each.

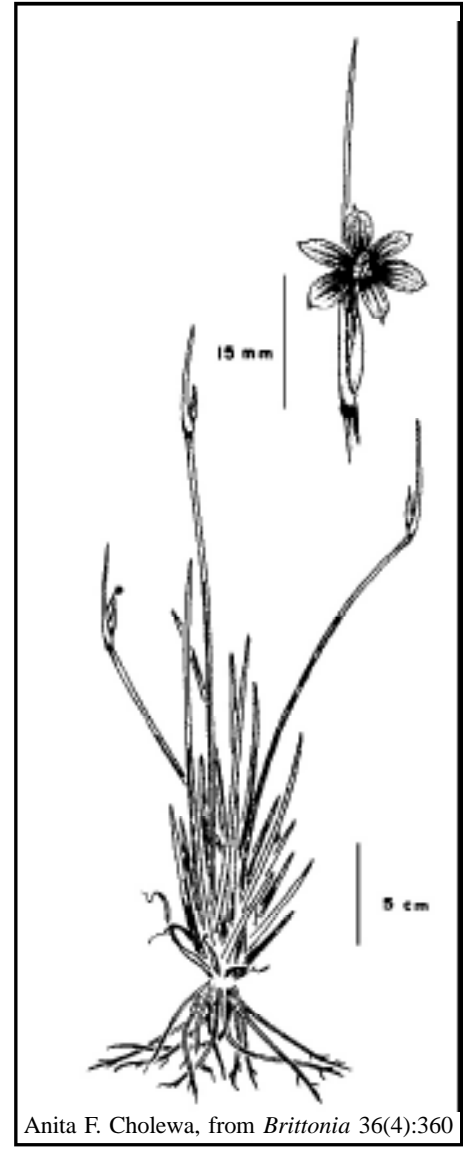

Figure G-15

DISTRIBUTION: Northern blue-eyed grass is distributed in the Great Plains from Alberta and Saskatchewan to Texas, and west of the continental divide in Idaho. In Montana, it is only known from the rolling Missouri Coteau area of Sheridan County, where it was collected east of Comertown. The more common Sisyrinchium montanum was documented in the eastern end of the County in Medicine Lake sandhills, alkali lake springs and peatlands, and around Brush Mountain.

HABITAT: This species is restricted to a low prairie or wet meadow settings. It was collected in Sheridan County from a prairie pothole margin in association with meadow anemone (Anemone canadensis) and meadow hawksbeard (Crepis runcinata).

COMMENTS: Northern blue-eyed grass is one of three species that was not relocated in the course of this study. It is among a group of species potentially benefiting from further survey in and restoration of wetland margins. It is the only plant species of concern in Sheridan County that is known from no other counties in Montana. 
Appendix J.

Flora of Sheridan County 


\section{Flora of Sheridan County}

\begin{tabular}{|c|c|c|c|}
\hline Scientific Name & Common Name & Origin & Family Code \\
\hline$\overline{\text { Abronia fragrans }}$ & Fragrant White Abronia & $\overline{\mathrm{N}}$ & "NYCTAG \\
\hline Abronia micrantha & Small-flowered Abronia & $\mathrm{N}$ & NYCTAG \\
\hline Acer negundo var interius & Box-elder & $\mathrm{N}$ & ACERAC \\
\hline Achillea millefolium var lanulosa & Common Yarrow & $\mathrm{N}$ & ASTERA \\
\hline Actaea rubra & Baneberry & $\mathrm{N}$ & RANUNC \\
\hline Agoseris cuspidata & Toothed Microseris & $\mathrm{N}$ & ASTERA \\
\hline Agoseris glauca var glauca & Pale Agoseris & $\mathrm{N}$ & ASTERA \\
\hline Agrimonia gryposepala & Common Agrimony & $\mathrm{N}$ & ROSACE \\
\hline Agropyron cristatum & Crested Wheatgrass & $\mathrm{E}$ & POACEA \\
\hline \multicolumn{4}{|c|}{$\begin{array}{l}\text { Agropyron dasystachyum - see Elymus } \\
\text { lanceolatus }\end{array}$} \\
\hline Agropyron repens & Quackgrass & $\mathrm{E}$ & POACEA \\
\hline \multicolumn{4}{|c|}{ Agropyron smithii - see Pascopyron smithii } \\
\hline \multicolumn{4}{|c|}{$\begin{array}{l}\text { Agropyron spicatum - see Pseudoroegneria } \\
\text { spicata }\end{array}$} \\
\hline Agrostis scabra & Tickle-grass & $\mathrm{N}$ & POACEA \\
\hline Agrostis stolonifera & Creeping Bentgrass & $\mathrm{E}$ & POACEA \\
\hline Alisma gramineum & Narrowleaf Waterplantain & $\mathrm{N}$ & ALISMA \\
\hline Alisma triviale & American Waterplantain & $\mathrm{N}$ & ALISMA \\
\hline Allium textile & Textile Onion & $\mathrm{N}$ & LILIAC \\
\hline Alopecurus aequalis & Short-awn Foxtail & $\mathrm{N}$ & POACEA \\
\hline Alyssum desortum & Desert Alyssum & $\mathrm{E}$ & BRASSI \\
\hline Amaranthus albus & Tumbleweed & $\mathrm{N}$ & AMARAN \\
\hline Amaranthus blitoides & Prostrate Pigweed & $\mathrm{N}$ & AMARAN \\
\hline Amaranthus graecizans & Prostrate Pigweed & $\mathrm{N}$ & AMARAN \\
\hline Amaranthus powellii & Powell's Amaranthus & $\mathrm{N}$ & AMARAN \\
\hline Amaranthus retroflexus & Rough Pigweed & $\mathrm{E}$ & AMARAN \\
\hline Ambrosia artemisiifolia & Annual Ragweed & $\mathrm{N}$ & ASTERA \\
\hline Ambrosia psilostachya & Western Ragweed & $\mathrm{N}$ & ASTERA \\
\hline Ambrosia trifida var trifida & Giant Ragweed & $\mathrm{N}$ & ASTERA \\
\hline Amelanchier alnifolia & Western Serviceberry & $\mathrm{N}$ & ROSACE \\
\hline Amelanchier sanguinea & Low Serviceberry & $\mathrm{N}$ & ROSACE \\
\hline Ammannia coccinea & Scarlet Ammannia & $\mathrm{N}$ & LYTHRA \\
\hline Andropogon gerardii & Big Bluestem & $\mathrm{N}$ & POACEA \\
\hline Andropogon hallii & Sandhill Bluestem & $\mathrm{N}$ & POACEA \\
\hline \multicolumn{4}{|c|}{$\begin{array}{l}\text { Andropogon scoparius - see Schizachyrium } \\
\text { scoparium }\end{array}$} \\
\hline Androsace occidentalis & Western Fairy-candelabra & $\mathrm{N}$ & PRIMUL \\
\hline Androsace septentrionalis & Pygmyflower Rockjasmine & $\mathrm{N}$ & PRIMUL \\
\hline Anemone canadensis & Meadow Anemone & $\mathrm{N}$ & RANUNC \\
\hline Anemone cylindrica & Candle Anemone & $\mathrm{N}$ & RANUNC \\
\hline Anemone multifida & Cliff Anemone & $\mathrm{N}$ & RANUNC \\
\hline
\end{tabular}


Flora of Sheridan County

Scientific Name

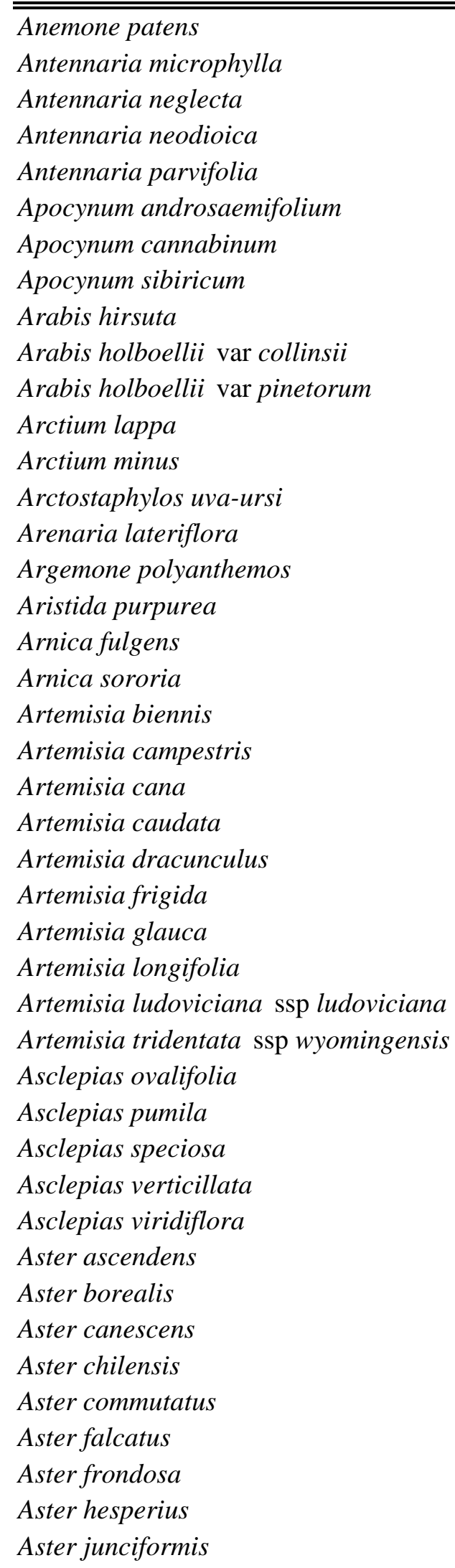

Common Name

Pasqueflower
Rosy Pussy-toes
Field Pussy-toes
Northern Pussy-toes
Nuttall's Pussy-toes
Spreading Dogbane
Hemp Dogbane
Clasping-leaved Dogbane
Hairy Rockcress
Holboell's Rockcress
Holboell's Rockcress
Great Burdock
Common Burdock
Kinnikinnick
Bluntleaf Sandwort
Prickly Poppy
Red Threeawn
Orange Arnica
Twin Arnica
Biennial Wormwood
Pacific Sagewort
Silver Sagebrush
Pacific Sagewort
Wormwood
Fringed Sagewort
Tarragon
Long-leaved Sagewort
Sagewort

Sagewort

Wyoming Big Sagebrush

Ovalleaf Milkweed

Plains Milkweed

Showy Milkweed

Whorled Milkweed

Green Milkweed

Long-leaved Aster

Rush Aster

Hoary Aster

Long-leaved Aster

White-prairie Aster

White-prairie Aster

Alkali Aster

Marsh Aster

Rush Aster
Origin Family Code

N RANUNC

$\mathrm{N}$ ASTERA

$\mathrm{N}$ ASTERA

$\mathrm{N}$ ASTERA

$\mathrm{N}$ ASTERA

$\mathrm{N}$ APOCYN

$\mathrm{N}$ APOCYN

$\mathrm{N}$ APOCYN

$\mathrm{N}$ BRASSI

$\mathrm{N}$ BRASSI

$\mathrm{N}$ BRASSI

E ASTERA

E ASTERA

$\mathrm{N}$ ERICAC

$\mathrm{N}$ CARYOP

N PAPAVE

$\mathrm{N}$ POACEA

$\mathrm{N}$ ASTERA

$\mathrm{N}$ ASTERA

$\mathrm{N}$ ASTERA

$\mathrm{N}$ ASTERA

$\mathrm{N}$ ASTERA

$\mathrm{N}$ ASTERA

$\mathrm{N}$ ASTERA

$\mathrm{N}$ ASTERA

$\mathrm{N}$ ASTERA

$\mathrm{N}$ ASTERA

$\mathrm{N}$ ASTERA

$\mathrm{N}$ ASTERA

$\mathrm{N}$ ASCLEP

$\mathrm{N}$ ASCLEP

$\mathrm{N}$ ASCLEP

$\mathrm{N}$ ASCLEP

$\mathrm{N}$ ASCLEP

$\mathrm{N}$ ASTERA

$\mathrm{N}$ ASTERA

$\mathrm{N}$ ASTERA

$\mathrm{N}$ ASTERA

$\mathrm{N}$ ASTERA

$\mathrm{N}$ ASTERA

$\mathrm{N}$ ASTERA

$\mathrm{N}$ ASTERA

$\mathrm{N}$ ASTERA 


\section{Flora of Sheridan County}

Scientific Name

\begin{tabular}{l}
\hline Aster laevis \\
Aster longifolius
\end{tabular}

Aster oblongifolius

Aster ptarmicoides - see Solidago

ptarmicoides

Astragalus aboriginum

Astragalus adsurgens

Astragalus agrestis

Astragalus americanus

Astragalus bisulcatus

Astragalus caespitosus

Astragalus canadensis

Astragalus ceramicus var filifolius

Astragalus crassicarpus var crassicarpus

Astragalus drummondii

Astragalus flexuosus

Astragalus geyeri

Astragalus gilviflorus

Astragalus gracilis

Astragalus longifolius

Astragalus lotiflorus

Astragalus missouriensis

Astragalus pectinatus

Astragalus purshii

Astragalus spatulatus

Astragalus striatus

Astragalus tenellus

Astragalus vexilliflexus

Atriplex argentea

Atriplex canescens

Atriplex confertifolia

Atriplex dioica - see A. suckleyi

Atriplex gardneri

Atriplex hastata

Atriplex hortensis

Atriplex patula

Atriplex powellii

Atriplex prostrata

Atriplex rosea

Atriplex suckleyi

Axyris amaranthoides

Bahia oppositifolia - see Picradeniopsis oppositifolia
Common Name

Origin Family Code

$\begin{array}{lll}\text { Smooth Aster } & \mathrm{N} & \text { ASTERA } \\ \text { Rush Aster } & \mathrm{N} & \text { ASTERA } \\ \text { Aromatic Aster } & \mathrm{N} & \text { ASTERA }\end{array}$

Indian Milk-vetch

Standing Milk-vetch

Field Milk-vetch

American Milk-vetch

Two-Groove Milk-vetch

Draba Milk-vetch

Canada Milk-vetch

Painted Milk-vetch

Ground Plum

Drummond's Milk-vetch

Wiry Milk-vetch

Geyer's Milkvetch

Plains Orophaca

Slender Milk-vetch

Painted Milk-vetch

Lotus Milk-vetch

Missouri Milk-vetch

Tine-leaved Milk-vetch

Pursh's Milk-vetch

Draba Milk-vetch

Standing Milk-vetch

Pulse Milk-vetch

Bent-flowered Milk-vetch

Silverscale

Four-wing Saltbush

Shadscale

N FABACE

N FABACE

N FABACE

$\mathrm{N}$ FABACE

$\mathrm{N}$ FABACE

N FABACE

$\mathrm{N}$ FABACE

$\mathrm{N}$ FABACE

$\mathrm{N}$ FABACE

$\mathrm{N}$ FABACE

$\mathrm{N}$ FABACE

N FABACE

$\mathrm{N}$ FABACE

$\mathrm{N}$ FABACE

$\mathrm{N}$ FABACE

$\mathrm{N}$ FABACE

$\mathrm{N}$ FABACE

$\mathrm{N}$ FABACE

$\mathrm{N}$ FABACE

$\mathrm{N}$ FABACE

$\mathrm{N}$ FABACE

$\mathrm{N}$ FABACE

$\mathrm{N}$ FABACE

$\mathrm{N}$ CHENOP

$\mathrm{N}$ CHENOP

$\mathrm{N}$ CHENOP

Gardner's Saltsage

Common Orache

$\mathrm{N}$ CHENOP

$\mathrm{N}$ CHENOP

Garden Orache

Fat-hen

Powell's Saltbush

Triangle Orache

Red Orache

Rillscale

Russian Pigweed
E CHENOP

$\mathrm{N}$ CHENOP

$\mathrm{N}$ CHENOP

$\mathrm{N}$ CHENOP

E CHENOP

$\mathrm{N}$ CHENOP

E CHENOP 


\section{Flora of Sheridan County}

Scientific Name

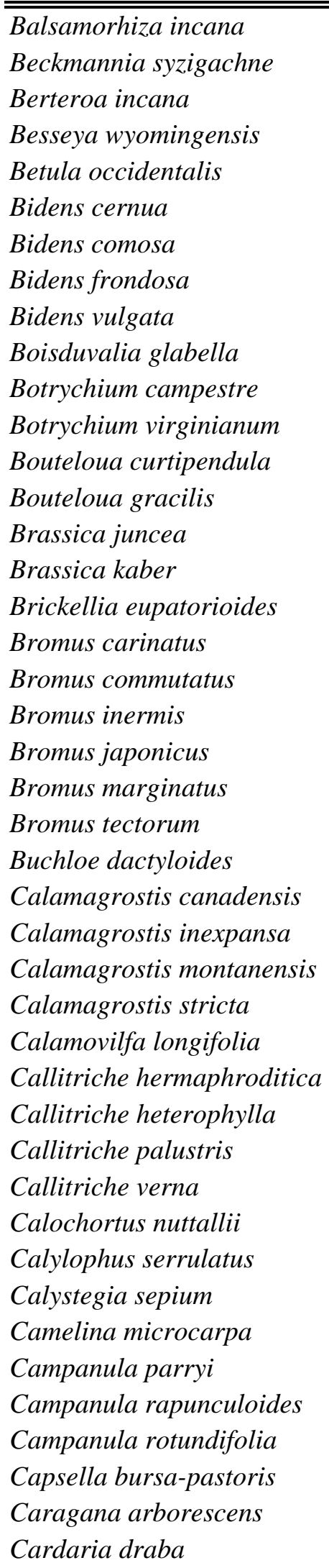

Common Name

Hoary Balsamroot
American Sloughgrass
Berteroa
Wyoming Kittentail
Water Birch
Nodding Beggar-ticks
Threelobed Beggar-ticks
Leafy Beggar-ticks
Tall Burr-marigold
Smooth Spike-primrose
Prairie Dunewort
Virginia Grape-fern
Sideoat Grama
Blue Grama
Indian Mustard
Wild Mustard
False-boneset
Mountain Brome
Hairy Chess
Smooth Brome
Japanese Brome
Mountain Brome
Cheatgrass
Buffalo Grass
Bluejoint Reedgrass
Northern Reedgrass
Plains Reedgrass
Narrow-spiked Reedgrass
Prairie Sandreed
Autumnal Water-starwort
Bolander's Waterstarwort
Spring Water-starwort
Spring Water-starwort
Sego-lilly
Plains Yellow Primrose
Hedge Bindweed
Smallseed False Flax
Parry's Bellflower
Creeping Bellflower
Harebell

Origin Family Code

N ASTERA

$\mathrm{N}$ POACEA

E BRASSI

N SCROPH

$\mathrm{N}$ BETULA

$\mathrm{N}$ ASTERA

$\mathrm{N}$ ASTERA

$\mathrm{N}$ ASTERA

$\mathrm{N}$ ASTERA

$\mathrm{N}$ ONAGRA

$\mathrm{N}$ OPHIOG

$\mathrm{N}$ OPHIOG

$\mathrm{N}$ POACEA

$\mathrm{N}$ POACEA

E BRASSI

E BRASSI

$\mathrm{N}$ ASTERA

$\mathrm{N}$ POACEA

E POACEA

E POACEA

E POACEA

N POACEA

E POACEA

$\mathrm{N}$ POACEA

$\mathrm{N}$ POACEA

$\mathrm{N}$ POACEA

$\mathrm{N}$ POACEA

$\mathrm{N}$ POACEA

$\mathrm{N}$ POACEA

$\mathrm{N}$ CALLIT

$\mathrm{N}$ CALLIT

$\mathrm{N}$ CALLIT

$\mathrm{N}$ CALLIT

$\mathrm{N}$ LILIAC

$\mathrm{N}$ ONAGRA

E CONVOL

E BRASSI

E CAMPAN

E CAMPAN

N CAMPAN

E BRASSI

E FABACE

E BRASSI 


\section{Flora of Sheridan County}

Scientific Name

Common Name

Origin Family Code

Carex aquatilis

Carex atherodes

Carex athrostachya

Carex aurea

Carex brevior

Carex cusickii

Carex deweyana

Carex douglasii

Carex duriuscula

Carex eburnea

Carex eleocharis - see C. duriuscula

Carex exsiccata

Carex filifolia

Carex heliophila - see C. inops

Carex inops

Carex laeviconica

Carex lanuginosa

Carex nebrascensis

Carex oederi - see C. viridula ssp viridula

Carex parryana

Carex pensylvanica

Carex praegracilis

Carex praticola

Carex rossii

Carex sprengelii

Carex stenophylla - see Carex duruiscula

Carex synchnocephala

Carex utriculata

Carex vesicaria var monile

Carex vesicaria var vesicaria

Carex viridula ssp viridula

Castilleja sessiliflora

Catabrosa aquatica

Celastrus scandens

Centaurea maculosa

Centaurea repens

Centunculus minimus

Cerastium arvense

Ceratoides lanata

Ceratoides lanata - see Krascheninnikovia lanata

Ceratophyllum demersum

Water Sedge
Awned Sedge
Slender-beaked Sedge
Golden Sedge
Short-beaked Sedge
Cusick's Sedge
Dewey's Sedge
Douglas's Sedge
Narrow-leaved Sedge
Ivory Sedge

Inflated Sedge

Thread-leaved Sedge

Sun sedge

Smooth Sedge

Woolly Sedge

Nebraska Sedge

Parry's Sedge

Penn's Sedge

Clustered Field Sedge

Meadow Sedge

Ross Sedge

Sprengel's Sedge

Many-headed Sedge

Beaked Sedge

Inflated Sedge

Inflated Sedge

Green Sedge

Downy Painted-cup

Brookgrass

American Bittersweet

Spotted Knapweed

Russian Knapweed

Chaffweed

Field Chickweed

Winterfat

Hornwort
CYPERA

CYPERA

$\mathrm{N}$ CYPERA

$\mathrm{N}$ CYPERA

$\mathrm{N}$ CYPERA

$\mathrm{N}$ CYPERA

$\mathrm{N}$ CYPERA

N CYPERA

$\mathrm{N}$ CYPERA

$\mathrm{N}$ CYPERA

N CYPERA

$\mathrm{N}$ CYPERA

$\mathrm{N}$

$\mathrm{N}$ CYPERA

$\mathrm{N}$ CYPERA

$\mathrm{N}$ CYPERA

N CYPERA

$\mathrm{N}$ CYPERA

$\mathrm{N}$ CYPERA

$\mathrm{N}$ CYPERA

$\mathrm{N}$ CYPERA

$\mathrm{N}$ CYPERA

N CYPERA

N CYPERA

$\mathrm{N}$ CYPERA

N CYPERA

$\mathrm{N}$ CYPERA

N SCROPH

$\mathrm{N}$ POACEA

$\mathrm{N}$ CELAST

E ASTERA

E ASTERA

N PRIMUL

$\mathrm{N}$ CARYOP

$\mathrm{N}$ CHENOP

$\mathrm{N}$

CERATO 


\section{Flora of Sheridan County}

\section{Scientific Name}

Common Name

Origin Family Code

Chaenactis douglasii

Chamaerhodos erecta

Chenopodium album

Chenopodium desiccatum

Chenopodium leptophyllum

Chenopodium pratericola

Chenopodium subglabrum

Chrysopsis villosa - see Heterotheca villosa

Chrysothamnus nauseosus ssp graveolens

Chrysothamnus nauseosus ssp nauseosus

Chrysothamnus nauseosus var petrophilus

Cicuta douglasii

Cicuta maculata

Cinna arundinacea

Cinna latifolia

Cirsium arvense

Cirsium canescens

Cirsium flodmanii

Cirsium pulcherrimum

Cirsium undulatum

Clematis ligusticifolia

Cleome serrulata

Collomia linearis

Comandra pallida

Comandra umbellata

Conringia orientalis

Convoluvus sepium - see Calystegia sepium

Convolvulus arvensis

Conyza canadensis

Corallorhiza striata

Coreopsis tinctoria

Cornus stolonifera

Corydalis aurea

Coryphantha missouriensis

Coryphantha vivipara

Crataegus chrysocarpa

Crepis atribarba

Crepis modocensis

Crepis occidentalis

Hoary Chaenactis
Chamaerhodos
Common Lambsquarters
Desert Goosefoot
Slimleaf Goosefoot
Slimleaf Goosefoot
Smooth Goosefoot

Smooth Goosefoot

Rubber Rabbitbrush

Rubber Rabbitbrush

Rubber Rabbitbrush

Douglas Water-hemlock

Spotted Water-hemlock

Woodreed

Drooping Woodreed

Canada Thistle

Platte Thistle

Flodman's Thistle

Prairie Thistle

Wavy-leaved Thistle

Western Virgins-bower

Rocky Mountain Bee Plant

Narrow-leaf Collomia

Pale Bastard Toad-flax

Bastard Toad-flax

Mustard Hare's Ear

$\begin{array}{ll}\mathrm{N} & \text { ASTERA } \\ \mathrm{N} & \text { ASTERA } \\ \mathrm{N} & \text { ASTERA } \\ \mathrm{N} & \text { APIACE } \\ \mathrm{N} & \text { APIACE } \\ \mathrm{N} & \text { POACEA } \\ \mathrm{N} & \text { POACEA } \\ \mathrm{E} & \text { ASTERA } \\ \mathrm{N} & \text { ASTERA } \\ \mathrm{N} & \text { ASTERA } \\ \mathrm{N} & \text { ASTERA } \\ \mathrm{N} & \text { ASTERA } \\ \mathrm{N} & \text { RANUNC } \\ \mathrm{N} & \text { CAPPAR } \\ \mathrm{N} & \text { POLEMO } \\ \mathrm{N} & \text { SANTAL } \\ \mathrm{N} & \text { SANTAL } \\ \mathrm{E} & \text { BRASSI }\end{array}$

Field Bindweed

Horseweed

Striped Coral-root

Plains Coreopsis

Red-osier Dogwood

Golden Corydalis

Nipple Coryphantha

Pincushion Cactus

Yellow-fruit Hawthorn

Slender Hawksbeard

Low Hawksbeard

Western Hawksbeard
ASTERA

$\mathrm{N}$ ROSACE

E CHENOP

$\mathrm{N}$ CHENOP

$\mathrm{N}$ CHENOP

$\mathrm{N}$ CHENOP

$\mathrm{N}$ CHENOP 


\section{Flora of Sheridan County}

Scientific Name

Crepis occidentalis

Crepis runcinata

Cryptantha celosioides

Cryptantha fendleri

Cryptantha minima

Cryptantha spiculifera

Cryptantha torreyana

Cuscuta coryli

Cuscuta pentagona

Cuscuta umbrosa

Cycloloma atriplicifolium

Cymopterus acaulis

Cymopterus terebinthinus

Cyperus schweinitzii

Cystopteris fragilis

Dalea candida

Dalea enneandra

Dalea purpurea

Dalea villosa

Danthonia californica

Datura stramonium

Delphinium bicolor

Deschampsia cespitosa

Descurainia pinnata

Descurainia richardsonii

Descurainia sophia

Disporum trachycaulum

Distichilis stricta - see D. spicata

Distichlis spicata

Dodecatheon pulchellum

Draba micrantha

Draba nemorosa

Draba reptans

Dyssodia papposa

Echinacea angustifolia

Echinacea pallida

Echinochloa crus-gallii

Echinochloa muricata

Echinocystis lobata

Elaeagnus angustifolia

Elaeagnus commutata

Eleocharis acicularis

Eleocharis calva
Common Name

Origin Family Code

Western Hawksbeard
Meadow Hawksbeard
Northern Cryptantha
Fendler's Cryptantha
Annual Cryptantha
Bristly Cryptantha
Torrey's Cryptantha
Hazel Dodder
Five-angled Dodder
Gronovius' Dodder
Tumble Ringwing
Plains Cymopterus
Turpentine Cymopterus
Schweinitz's Flatsedge
Brittle Bladder-fern
White Prairie-clover
Nine-anther Prairie-clover
Purple Prairie-clover
Silky Prairie-clover
Timber Oatgrass
Thorn Apple
Little Larkspur
Tufted Hairgrass
Western Tansymustard
Richardson's Tansymustard
Flixweed
Fairy-bell

Alkali Saltgrass

Few-flowered Shooting Star

Carolina Whitlow-grass

Woods Draba

Carolina Whitlow-grass

Fetid Marigold

Pale Purple Coneflower

Pale Purple Coneflower

Large Barnyard Grass

Pointed Barnyard-grass

Wild Cucumber

Russian Olive

Silverberry

Needle Spike-rush

Common Spike-rush
ASTERA

ASTERA

$\mathrm{N}$ BORAGI

$\mathrm{N}$ BORAGI

$\mathrm{N}$ BORAGI

$\mathrm{N}$ BORAGI

$\mathrm{N}$ BORAGI

$\mathrm{N}$ CUSCUT

$\mathrm{N}$ CUSCUT

$\mathrm{N}$ CUSCUT

$\mathrm{N}$ CHENOP

$\mathrm{N}$ APIACE

$\mathrm{N}$ APIACE

$\mathrm{N}$ CYPERA

$\mathrm{N}$ POLYPO

$\mathrm{N}$ FABACE

$\mathrm{N}$ FABACE

$\mathrm{N}$ FABACE

$\mathrm{N}$ FABACE

$\mathrm{N}$ POACEA

$\mathrm{N}$ SOLANA

N RANUNC

E POACEA

$\mathrm{N}$ BRASSI

$\mathrm{N}$ BRASSI

E BRASSI

$\mathrm{N}$ LILIAC

N POACEA

N PRIMUL

$\mathrm{N}$ BRASSI

$\mathrm{N}$ BRASSI

$\mathrm{N}$ BRASSI

$\mathrm{N}$ ASTERA

$\mathrm{N}$ ASTERA

$\mathrm{N}$ ASTERA

E POACEA

E POACEA

N CUCURB

E ELAEAG

$\mathrm{N}$ ELAEAG

N CYPERA

$\mathrm{N}$ CYPERA 


\section{Flora of Sheridan County}

\begin{tabular}{|c|c|c|c|}
\hline Scientific Name & Common Name & Origin & Family Co \\
\hline Eleocharis erythropoda & Reddish Spike-rush & $\mathrm{N}$ & CYPERA \\
\hline Eleocharis macrostachya & Large-spike Spike-rush & $\mathrm{N}$ & CYPERA \\
\hline Eleocharis palustris & Common Spike-rush & $\mathrm{N}$ & CYPERA \\
\hline \multicolumn{4}{|c|}{ Eleocharis pauciflora - see E. quinqueflora } \\
\hline Eleocharis quinqueflora & Few-flowered Spikerush & $\mathrm{N}$ & CYPERA \\
\hline Ellisia nyctelea & Nyctelea & $\mathrm{N}$ & HYDROP \\
\hline Elodea canadensis & Canada Waterweed & $\mathrm{N}$ & HYDROC \\
\hline Elymus canadensis & Canada Wildrye & $\mathrm{N}$ & POACEA \\
\hline Elymus elymoides & Bottlebrush Squirreltail & $\mathrm{N}$ & POACEA \\
\hline Elymus lanceolatus & Thick-spike Wheatgrass & $\mathrm{N}$ & POACEA \\
\hline Elymus macounii & Macoun Wildrye & $\mathrm{N}$ & POACEA \\
\hline Elymus smithii & Western Wheatgrass & $\mathrm{N}$ & POACEA \\
\hline Elymus spicatus & Bluebunch Wheatgrass & $\mathrm{N}$ & POACEA \\
\hline Elymus trachycaulus & Bearded Wheatgrass & $\mathrm{N}$ & POACEA \\
\hline Elymus virginicus & Virginia Wildrye & $\mathrm{N}$ & POACEA \\
\hline Epilobium angustifolium & Fireweed & $\mathrm{N}$ & ONAGRA \\
\hline Epilobium ciliatum & Common Willow-herb & $\mathrm{N}$ & ONAGRA \\
\hline Epilobium leptophyllum & Narrow-leaved Willow-herb & $\mathrm{N}$ & ONAGRA \\
\hline Epilobium paniculatum & Autumn Willow-herb & $\mathrm{N}$ & ONAGRA \\
\hline Equisetum arvense & Field Horsetail & $\mathrm{N}$ & EQUISE \\
\hline Equisetum hyemale & Common Scouring-rush & $\mathrm{N}$ & EQUISE \\
\hline Equisetum hyemale & Common Scouring-rush & $\mathrm{N}$ & EQUISE \\
\hline Equisetum kansanum & Smooth Scouring-rush & $\mathrm{N}$ & EQUISE \\
\hline Equisetum laevigatum & Smooth Scouring-rush & $\mathrm{N}$ & EQUISE \\
\hline Equisetum praealtum & Common Scouring-rush & $\mathrm{N}$ & EQUISE \\
\hline Eragrostis cilianensis & Stinkgras & $\mathrm{E}$ & POACEA \\
\hline Eragrostis pectinacea & Purple Lovegrass & $\mathrm{N}$ & POACEA \\
\hline Erigeron asper & Smooth Daisy & $\mathrm{N}$ & ASTERA \\
\hline Erigeron caespitosus & Tufted Fleabane & $\mathrm{N}$ & ASTERA \\
\hline Erigeron canadensis & Horseweed & $\mathrm{N}$ & ASTERA \\
\hline Erigeron compositus & Cut-leaved Daisy & $\mathrm{N}$ & ASTERA \\
\hline Erigeron divergens & Spreading Fleabane & $\mathrm{N}$ & ASTERA \\
\hline Erigeron glabellus & Smooth Daisy & $\mathrm{N}$ & ASTERA \\
\hline Erigeron glabellus & Smooth Daisy & $\mathrm{N}$ & ASTERA \\
\hline Erigeron philadelphicus & Philadelphia Fleabance & $\mathrm{N}$ & ASTERA \\
\hline Erigeron pumilus & Shaggy Fleabane & $\mathrm{N}$ & ASTERA \\
\hline Erigeron strigosus & Branching Daisy & $\mathrm{N}$ & ASTERA \\
\hline Eriogonum аппиит & Annual Buckwheat & $\mathrm{N}$ & POLYGO \\
\hline Eriogonum flavum & Yellow Buckwheat & $\mathrm{N}$ & POLYGO \\
\hline Eriogonum multiceps & $\begin{array}{l}\text { Few-flowered Wild } \\
\text { Buckwheat }\end{array}$ & $\mathrm{N}$ & POLYGO \\
\hline
\end{tabular}




\section{Flora of Sheridan County}

Scientific Name

Eriogonum pauciflorum

Eriophorum angustifolium

Eriophorum polystachion

Erucastrum gallicum

Erysimum asperum

Erysimum capitatum

Erysimum cheiranthoides

Erysimum inconspicuum

Erysimum repandrum

Euphorbia cyparissias

Euphorbia esula

Euphorbia glyptosperma

Euphorbia marginata

Euphorbia missurica

Euphorbia robusta

Euphorbia serpyllifolia

Euphorbia spathulata

Eustoma grandiflorum

Euthamia occidentalis

Festuca arundinacea

Festuca octoflora

Festuca ovina

Fragaria virginiana

Fraxinus pennsylvanica

Fumaria officinalis

Gaillardia aristata

Galium aparine

Galium boreale

Galium triflorum

Gaura coccinea

Gaura parviflora

Gayophytum diffusum

Gentiana affinis

Gentianella amarella

Geum aleppicum

Geum canadense

Geum macrophyllum

Geum triflorum

Gilia congesta

Glaux maritima

Glyceria grandis

Glyceria striata
Common Name

Few-flowered Wild

Buckwheat

Many-spiked Cotton-grass

Many-spiked Cotton-grass

Dog Mustard

Plains Wallflower

Western Wallflower

Wormseed Wallflower

Smallflowered Wallflower

Spreading Wallflower

Cypress Spurge

Leafy Spurge

Corrugate-seeded Spurge

Snow-on-the-mountain

Missouri Spurge

Rocky Mountain Spurge

Thyme-leaf Spurge

Spatulate-leaved Spurge

Showy Prairie-gentian

Western Goldenrod

Tall Fescue

Six-weeks Fescue

Sheep Fescue

Virginia Strawberry

Green Ash

Common Fumitory

Blanket-flower

Goose-grass

Northern Bedstraw

Sweetscented Bedstraw

Scarlet Gaura

Small-flowered Gaura

Spreading Groundsmoke

Pleated Gentian

Northern Gentian

Yellow Avens

White Avens

Large-leaved Avens

Prairie Smoke

Ballhead Gilia

Saltwort

American Mannagrass

Fowl Mannagrass
Origin Family Code

N POLYGO

$\mathrm{N}$ CYPERA

$\mathrm{N}$ CYPERA

E BRASSI

$\mathrm{N}$ BRASSI

$\mathrm{N}$ BRASSI

$\mathrm{N}$ BRASSI

$\mathrm{N}$ BRASSI

$\mathrm{N}$ BRASSI

E EUPHOR

E EUPHOR

$\mathrm{N}$ EUPHOR

$\mathrm{N}$ EUPHOR

$\mathrm{N}$ EUPHOR

$\mathrm{N}$ EUPHOR

$\mathrm{N}$ EUPHOR

$\mathrm{N}$ EUPHOR

$\mathrm{N}$ GENTIA

$\mathrm{N}$ ASTERA

E POACEA

$\mathrm{N}$ POACEA

$\mathrm{N}$ POACEA

N ROSACE

$\mathrm{N}$ OLEACE

E FUMARI

$\mathrm{N}$ ASTERA

$\mathrm{N}$ RUBIAC

$\mathrm{N}$ RUBIAC

$\mathrm{N}$ RUBIAC

$\mathrm{N}$ ONAGRA

$\mathrm{N}$ ONAGRA

$\mathrm{N}$ ONAGRA

$\mathrm{N}$ GENTIA

$\mathrm{N}$ GENTIA

$\mathrm{N}$ ROSACE

$\mathrm{N}$ ROSACE

N ROSACE

$\mathrm{N}$ ROSACE

$\mathrm{N}$ POLEMO

$\mathrm{N}$ PRIMUL

$\mathrm{N}$ POACEA

$\mathrm{N}$ POACEA 
Flora of Sheridan County

\section{Scientific Name}

\begin{tabular}{l}
\hline \hline Glycyrrhiza lepidota \\
Gnaphalium palustre \\
Grindelia squarrosa \\
Gutierrezia sarothrae \\
Gypsophila paniculata \\
Habenaria hyperborea \\
Hackelia deflexa \\
Hackelia floribunda \\
Haplopappus armerioides \\
Haplopappus lanceolatus \\
Haplopappus lanuginosus \\
Haplopappus spinulosus \\
Hedeoma drummondii \\
Hedeoma hispida \\
Hedysarum boreale \\
Helenium autumnale \\
Helianthus annuus \\
Helianthus maximiliani \\
Helianthus nuttallii \\
Helianthus pauciflorus \\
Helianthus petiolaris \\
Helianthus rigidus \\
Helianthus tuberosus \\
Helictotrichon hookeri \\
Heliotropium curassavicum \\
Heracleum spondylium \\
Hesperis matronalis \\
Heterotheca villosa \\
Heuchera richardsonii \\
Hieracium canadense \\
Hieracium umbellatum \\
Hordeum brachyantherum \\
Hordeum jubatum \\
Hordeum pusillum \\
Humulus lupulus \\
Hymenopappus filifolius \\
Hymenoxys acaulis \\
Hymenoxys richardsonii \\
Hyoscyamus niger \\
Hypericum scouleri \\
Ivomopsis congesta \\
Iva xanthifolia \\
\end{tabular}

Common Name

Origin Family Code

American Licorice
Lowl Cudweed
Curlycup Gumweed
Broom Snakeweed
Baby's Breath
Northern Green Bog-orchid
Nodding Stickseed
Showy Stickseed
Thrift Goldenweed

$\mathrm{N}$ FABACE

$\mathrm{N}$ ASTERA

$\mathrm{N}$ ASTERA

$\mathrm{N}$ ASTERA

E CARYOP

$\mathrm{N}$ ORCHID

$\mathrm{N}$ BORAGI

$\mathrm{N}$ BORAGI

$\mathrm{N}$ ASTERA

Lance-leaf Goldenweed N ASTERA

Woolly Goldenweed N ASTERA

Spiny Goldenweed

Drummond False Pennyroyal

Rough Pennyroyal

Northern Hedysarum

Sneezeweed

Common Sunflower

Maximilan's Sunflower

Nuttall's Sunflower

Showy Sunflower

Prairie Sunflower

Showy Sunflower

Jerusalem Artichoke

Spike Oat

Salt Heliotrope

Cow-parsnip

Dame's Rocket

Hairy Golden-aster

Richardson's Alumroot

Canada Hawkweed

Narrow-leaved Hawkweed

Meadow Barley

Foxtail Barley

Little Barley

Common Hop

Columbia Cut-leaf

Stemless Hymenoxys

Richardson's Hymenoxys

Black Henbane

Norton's St. Johnswort

Ballhead Gilia

Poverty-weed

Tall Marsh-elder

$\mathrm{N}$ ASTERA

$\mathrm{N}$ LAMIAC

$\mathrm{N}$ LAMIAC

$\mathrm{N}$ FABACE

$\mathrm{N}$ ASTERA

$\mathrm{N}$ ASTERA

$\mathrm{N}$ ASTERA

$\mathrm{N}$ ASTERA

$\mathrm{N}$ ASTERA

$\mathrm{N}$ ASTERA

$\mathrm{N}$ ASTERA

$\mathrm{N}$ ASTERA

$\mathrm{N}$ POACEA

$\mathrm{N}$ BORAGI

$\mathrm{N}$ APIACEA

E BRASSIC

$\mathrm{N}$ ASTERA

$\mathrm{N}$ SAXIFR

$\mathrm{N}$ ASTERA

$\mathrm{N}$ ASTERA

$\mathrm{N}$ POACEA

$\mathrm{N}$ POACEA

$\mathrm{N}$ POACEA

$\mathrm{N}$ CANNAB

$\mathrm{N}$ ASTERA

$\mathrm{N}$ ASTERA

$\mathrm{N}$ ASTERA

E SOLANA

$\mathrm{N}$ CLUSIA

$\mathrm{N}$ POLEMO

$\mathrm{N}$ ASTERA

$\mathrm{N}$ ASTERA 
Flora of Sheridan County

\section{Scientific Name}

\begin{tabular}{|c|c|c|c|}
\hline Juncus articulatus & Jointed Rush & $\mathrm{N}$ & JUNCAC \\
\hline Juncus balticus & Baltic Rush & $\mathrm{N}$ & JUNCAC \\
\hline Juncus bufonius & Toad Rush & $\mathrm{N}$ & JUNCAC \\
\hline Juncus dudleyi & Dudley Rush & $\mathrm{N}$ & JUNCAC \\
\hline Juncus longistylis & Long-styled Rush & $\mathrm{N}$ & JUNCAC \\
\hline Juncus nodosus & Tuberous Rush & $\mathrm{N}$ & JUNCAC \\
\hline Juncus tenuis & Slender Rush & $\mathrm{N}$ & JUNCAC \\
\hline Juncus torreyi & Torrey's Rush & $\mathrm{N}$ & JUNCAC \\
\hline Juniperus communis & Common Juniper & $\mathrm{N}$ & CUPRES \\
\hline Juniperus horizontalis & Creeping Juniper & $\mathrm{N}$ & CUPRES \\
\hline Juniperus scopulorum & Rocky Mountain Juniper & $\mathrm{N}$ & CUPRES \\
\hline \multicolumn{4}{|c|}{ Koeleria cristata - see $K$. macrantha } \\
\hline Koeleria pyramidata & Junegrass & $\mathrm{N}$ & POACEA \\
\hline Krascheninnikovia lanata & Winterfat & $\mathrm{N}$ & CHENOP \\
\hline Kuhnia eupatorioides & False-boneset & $\mathrm{N}$ & ASTERA \\
\hline Lactuca ludoviciana & Western Lettuce & $\mathrm{N}$ & ASTERA \\
\hline Lactuca oblongifolia & Blue Lettuce & $\mathrm{N}$ & ASTERA \\
\hline Lactuca serriola & Prickly Lettuce & $\mathrm{E}$ & ASTERA \\
\hline Lappula cenchrusoides & Millet Stickseed & $\mathrm{N}$ & BORAGI \\
\hline Lappula echinata & Europen Sticktight & $\mathrm{E}$ & BORAGI \\
\hline Lappula occidentalis & Desert Stickseed & $\mathrm{N}$ & BORAGI \\
\hline Lappula redowskii & Western Stickseed & $\mathrm{N}$ & BORAGI \\
\hline Lemna minor & Common Duckweed & $\mathrm{N}$ & LEMNAC \\
\hline Lemna trisulca & Star Duckweed & $\mathrm{N}$ & LEMNAC \\
\hline Leonurus cardiaca & Motherwort & $\mathrm{E}$ & LAMIAC \\
\hline Lepidium densiflorum & Prairie Pepperweed & $\mathrm{N}$ & BRASSI \\
\hline Lepidium perfoliatum & Clasping Pepperweed & $\mathrm{E}$ & BRASSI \\
\hline Lepidium ramosissimum & Branched Pepperweed & $\mathrm{N}$ & BRASSI \\
\hline Lepidium virginicum & Tall Pepperweed & $\mathrm{N}$ & BRASSI \\
\hline Leptochloa fascicularis & Loose-flowered Sprangletop & $\mathrm{N}$ & POACEA \\
\hline Lesquerella alpina & Alpine Bladderpod & $\mathrm{N}$ & BRASSI \\
\hline Lesquerella arenosa & Sand Bladderpod & $\mathrm{N}$ & BRASSI \\
\hline Lesquerella ludoviciana & Silvery Bladderpod & $\mathrm{N}$ & BRASSI \\
\hline Leucocrinum montanum & Mountain Lily & $\mathrm{N}$ & LILIAC \\
\hline Liatris ligulistylis & Large-headed Gay-feather & $\mathrm{N}$ & ASTERA \\
\hline Liatris punctata & Dotted Blazing-star & $\mathrm{N}$ & ASTERA \\
\hline Lilium philadelphicum & Motherwort & $\mathrm{N}$ & LAMIAC \\
\hline Limosella aquatica & Mudwort & $\mathrm{N}$ & SCROPH \\
\hline Linaria dalmatica & Dalmatian Toadflax & $\mathrm{E}$ & SCROPH \\
\hline Linum lewisii & Wild Blue Flax & $\mathrm{N}$ & LINACE \\
\hline Linum rigidum & Yellow Flax & $\mathrm{N}$ & LINACE \\
\hline Linum usitatissimum & Cultivated Flax & $\mathrm{E}$ & LINACE \\
\hline Lithospermum arvense & Corn Gromwell & $\mathrm{E}$ & BORAGI \\
\hline
\end{tabular}




\section{Flora of Sheridan County}

Scientific Name

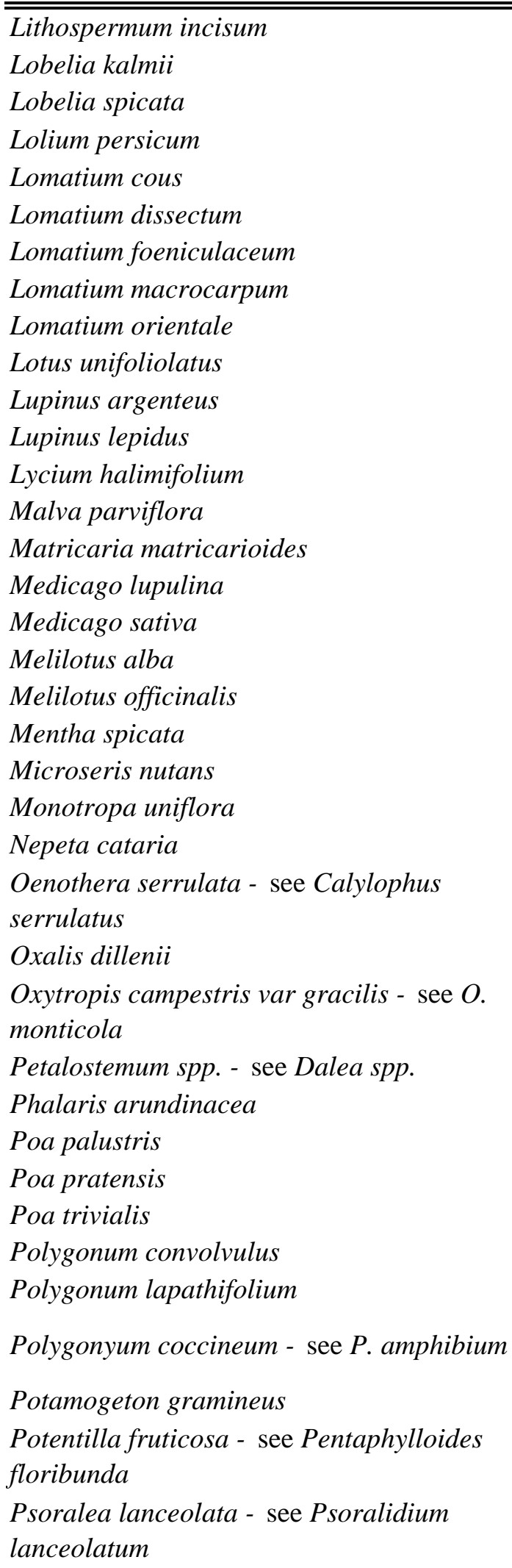

Common Name

Yellow Gromwell
Kalm's Lobelia
Pale-spike Lobelia
Persian Darnel
Cous Biscuit-root
Fern-leaved Desert-parsley
Fennel-leaved Desert-parsley
Bigseed Desert-parsley
Oriental Lomatium
Spanish-clover
Silvery Lupine
Prairie Lupine
Matrimonyvine
Little Mallow
Pineapple Weed
Black Medic
Alfalfa
White Sweetclover
Yellow Sweetclover
Spearmint
Nodding Microseris
Indian Pipe
Catnip

Dillen's Wood-sorrel

Reed Canarygrass

Fowl Bluegrass

Kentucky Bluegrass

Roughstalk Bluegrass

Black Bindweed

Willow Weed

Grass-leaved Pondweed
N OXALID

N OXALID

Origin Family Code

$\mathrm{N}$

$\mathrm{N}$

$\mathrm{N}$ CAMPAN

$\mathrm{N}$ CAMPAN

E POACEA

$\mathrm{N}$ APIACE

$\mathrm{N}$ APIACE

$\mathrm{N}$ APIACE

$\mathrm{N}$ APIACE

$\mathrm{N}$ APIACE

$\mathrm{N}$ FABACE

$N$ FABACE

$\mathrm{N}$ FABACE

E SOLANA

E MALVAC

$\mathrm{N}$ ASTERA

E FABACE

E FABACE

E FABACE

E FABACE

E LAMIAC

$\mathrm{N}$ ASTERA

$\mathrm{N}$ ERICAC

E LAMIAC

E POACEA

E POACEA

E POACEA

E POACEA

E POLYGO

E POLYGO

N POTAMO 


\section{Flora of Sheridan County}

\section{Scientific Name}

Ranunculus gmelinii

Rhamnus cathartica

Rosa acicularis - see R. sayi

Rumex acetosa

Rumex crispus

Ruppia maritima

Salsola collina

Salsola iberica

Scirpus microcarpus

Scolochloa festucacea

Setaria viridis

Silene cserei

Sisymbrium altissimum

Sonchus arvensis

Sonchus asper

Sonchus uliginosus

Spergularia marina

Sphenopholis obtusata

Spirodela polyrhiza

Stellaria media

Stipa curtiseta

Stipa spartea var. curtiseta - see Stipa

curtiseta

Stipa viridula - see Nasella viridula

Suaeda depressa - see S. calciformis

Tanacetum vulgare

Taraxacum officinale

Thlaspi arvense

Tragopogon dubius

Trifolium pratense

Trifolium repens

Triodanis perfoliata

Ulmus pumila

Vaccaria pyramidata

Verbascum blattaria

Verbena hastata

Zygophyllum fabago

\section{Common Name}

Small Yellow Water-buttercup

European Buckthorn

Meadow Sorrel

Curly Dock

Wigeon-grass

Slender Russian Thistle

Russian Thistle

Small-flowered Bulrush

Whitetop

Green Foxtail

Smooth Catchfly

Tall Tumbleustard

Perennial Sowthistle

Sping Sowthistle

Marsh Sowthistle

Salt-marsh Sandspurry

Slender Wedgegrass

Greater Duckweed

Chickweed

Northern Porcupinegrass

\section{Origin Family Code}

N RANUNC

E RHAMNA

E POLYGO

E POLYGO

N RUPPIA

E CHENOP

E CHENOP

$\mathrm{N}$ CYPERA

$\mathrm{N}$ POACEA

E POACEA

E CARYOP

E BRASSI

E ASTERA

E ASTERA

E ASTERA

E CARYOP

$\mathrm{N}$ POACEAE

$\mathrm{N}$ LEMNAC

E CAROPHY

N POACEAE

Common Tansy
Common Dandelion
Field Pennycress
Goatsbeard
Red Clover
White Clover
Western Venus'-looking-glass
Siberian Elm
Cowcockle
Moth Mullein
Blue Verbena
Bean Caper

$\begin{array}{ll}\text { E } & \text { ASTERA } \\ \text { E } & \text { ASTERA } \\ \text { E } & \text { BRASSI } \\ \text { E } & \text { ASTERA } \\ \text { E } & \text { FABACE } \\ \text { E } & \text { FABACE } \\ & \\ N & \text { CAMPAN } \\ \text { E } & \text { ULMACE } \\ \text { E } & \text { CARYOP } \\ \text { E } & \text { SCROPH } \\ N & \text { VERBEN } \\ \text { E } & \text { ZYGOPH }\end{array}$

